\title{
Thickness-induced violation of de Haas-van Alphen effect through exact analytical solutions at a one-electron and a one-Composite Fermion level
}

\author{
G. Konstantinou ${ }^{1}$ and K. Moulopoulos ${ }^{2 *}$ \\ Department of Physics, University of Cyprus, PO Box 20537, 1678 Nicosia, Cyprus \\ 'ph06kg1@ucy.ac.cy, ${ }^{2} \cos @ u c y . a c . c y$
}

\begin{abstract}
A systematic study of the energetics of electrons in an interface in a magnetic field is reported with exact analytical calculations based on a Landau Level (LL) picture, by serious consideration of the finite thickness of the Quantum Well $(\mathrm{QW})$. The approach is physically transparent and subtly different in its line of reasoning from standard methods avoiding any semi-classical approximation. We find "internal" phase transitions (at partial LL filling) for magnetisation and susceptibility that are not captured by other approaches and that give rise to nontrivial violations of the standard de Haas-van Alphen periods, in a manner that reproduces the exact quantal astrophysical behaviours in the limit of full three-dimensional (3D) space. Upon inclusion of Zeeman splitting, additional features are also found, such as global energy minima originating from the interplay of QW, Zeeman and LL Physics, while a corresponding calculation in a Composite Fermion picture with $\Lambda$-Levels, leads to new predictions on magnetic properties of an interacting electron liquid. By pursuing the same line of reasoning for a topologically nontrivial system with a relativistic spectrum, we find evidence that similar effects might be operative in the dimensionality crossover of $3 \mathrm{D}$ strong topological insulators to $2 \mathrm{D}$ topological insulator quantum wells.
\end{abstract}

SHORT TITLE: Thickness-induced violation of de Haas-van Alphen effect

PACS numbers: 75.70.Cn, 71.70.Di, 73.20.-r, 73.63.Hs, 71.18.+y

\section{Introduction}

Recently, there has been a surge of interest in the new area of topological insulators [1,2], namely electronic systems characterised by a bulk insulating gap but also possessing topologically-protected gapless edge (or surface) states, i.e., dissipationless conducting surface modes, immune to nonmagnetic impurity scattering and geometrical defects. The simplest example of such a phase with broken time-reversal symmetry, can be found in a two-dimensional (2D) electron gas under a strong perpendicular magnetic field in the Quantum Hall regime. Through a very general bulk-edge correspondence [3], it has been well established that the number of dissipationless edge states is equal to the integer that arises from the so-called TKNN invariant [4], or the $1^{\text {st }}$ Chern number in a fibre bundle language [5], of the occupied energy bands. This is a bulk property related to the "vorticity" of the wavefunctions in the magnetic Brillouin zone. In a jellium model picture, the $1^{\text {st }}$ Chern number or the number of edge states, turns out to be equal to the number of completely filled Landau Levels (LLs) in the Integral Quantum Hall Effect (IQHE) regime. If one wanted to include the $3^{\text {rd }}$ dimension, i.e., to take into account the thickness of the macroscopic quasi-2D sample (interface or film) with open (rigid) boundary conditions, then a treatment of the above mathematical (topological) properties would be a formidable task. In fact, it would spoil the beauty of the standard topological arguments normally applied to the 2D Brillouin zone. Here, we point to an alternative general procedure that is rigorous and based on physical rather than purely mathematical arguments and that seems to have not been discussed in the past. It is based on energy interplays in a one-electron (or one-Composite Fermion) picture, leading to the possibility and in fact, showing the existence of abrupt changes in the occupancy of transverse, i.e., thickness-related modes in the ground state. These occur at partial LL filling and are accompanied by associated changes in thermodynamic and also possibly in transport properties; changes that, as it turns out, happen to occur in an interesting, although in a certain sense, nonintegrable fashion as the thickness is varied.

The method we are presenting is a canonical ensemble approach (fixed number of particles), which is subtly different from standard canonical or grandcanonical approaches that at some point invoke semi-classical approximations and that usually have mathematical difficulty in dealing exactly with the zero-temperature limit, 
i.e., it does not anticipate or assume a Fermi sphere in the $3 \mathrm{D}$ zero-field limit as part of the quasi- $2 \mathrm{D}$ calculation but naturally derives it in a direct and rigorous manner. The method is exact, involving no approximations whatsoever and it describes the zero-temperature case, although this is immediately generalisable if Fermi factors are included. What is most important is that it is physically transparent at every step of the procedure and hence, rather easy to use for other systems that are more involved or exotic. The method works directly in $k$ space by taking careful advantage of anisotropies in different directions by not using at all the density of states (DOS). The DOS is the key quantity in all other approaches through which, by reducing everything to the energy variable, basically masks the Physics (i.e., the intermediate physical steps) that take place in $k$-space and that depend on the geometry of each system. It is also not necessary to go through the rather difficult step of first finding the DOS by determining the exact energy spectrum. This is advantageous, especially if we want to have as much analytical control on our solution as possible. Moreover, a physical criterion (of "equilibrium") applied to the occupation procedure of a strongly anisotropic system is shown from the results to be superior to the usual semi-classical treatments that lead to the standard "magnetic oscillations" [6]. Unlike those methods, the present approach leads to exact quantal violations of the de Haas-van Alphen (dHvA) periodicities in the quasi-2D interface or film, which become smooth quantal deviations (from the dHvA periodicity) in the 3D limit.

The method can actually be useful in a wide range of applications because the precise role of thickness in various quasi-2D systems seems to be currently attracting considerable attention. By way of an example, mention should be made of bulk Quantum Hall Effect (QHE) measurements in a 3D topological insulator [7] where Shubnikovde Haas oscillations in highly doped $\mathrm{Bi}_{2} \mathrm{Se}_{3}$ give evidence for layered transport of bulk carriers, in which the sample thickness plays an essential role on the quantisation of magnetotransport but also of the more exciting thickness-related issue of 2D to 3D dimensionality crossover in topological insulators (an issue that is actually briefly touched upon in this paper, as will be seen shortly). However, in the bulk of this work, we take a step back and present the method in the simplest possible but still nontrivial setting. We solve exactly thicknessrelated problems involving an electron gas system in the jellium model, both with and without a magnetic field in various dimensionalities, demonstrating that even in these simplest possible cases, the role of thickness is nontrivial and noteworthy. [The jellium model gives the luxury of dealing with simple LLs, where their number is automatically identified with the topological (Chern) number or the number of edge states (whenever the LLs are completely filled). This gives one the opportunity to identify possible abrupt changes in the Chern number (when LLs are abruptly depopulated - as will actually occur many times in this work) with possible interesting consequences on transport properties. However, these deserve a separate article, as this one focuses on thermodynamic consequences, i.e., violations of dHvA periods.] Furthermore, because the largest part of our analysis utilises a jellium model of electrons in extended states, mention should also be made of a 2D semimetal that has recently been observed in wide $\mathrm{HgTe}$ quantum wells (QWs) with a broad range of interesting properties [8] and with their thickness still being an important factor not yet seriously studied. Moreover, very recent works on the 5/2-Fractional Quantum Hall Effect (FQHE) [9-11] examine the stability of the effect in wide QWs against the variation of their thickness and find anomalous features. It is with this in mind that we have applied the same method by carrying out a thickness-adapted Composite Fermion calculation, as will be seen shortly. Mention could also be made of recently studied highly quantum-confined nanoscale membranes, the thickness of which is crucial for their (mostly optical) properties [12], as well as of the newly discovered almost free electron gases in oxide heterointerfaces [13]. Finally, returning to the one-body Physics of the recently discovered topological insulators, our approach and results might actually cast doubt on the completeness of recent findings on a simple oscillatory crossover from a $2 \mathrm{D}$ to a $3 \mathrm{D}$ topological insulator [14], where transitions between different $z$-modes (with $z$ being the direction of the external magnetic field) may not have been treated entirely properly. This will be apparent from the present article - the point being that, in that work, energy comparisons are made under the assumption of a given (fixed) transverse mode, not taking into account the energetically favourable possibility of abrupt changes of such modes that might occur in nontrivial ways as the thickness is varied. As we will see in a preliminary study towards the end of this paper, although such transitions might occur at points located a little further than the $\Gamma$-point in the Brillouin zone, their distance from the $\Gamma$-point in $k$-space is actually quite small, such that these effects might be operative. We will actually see that they might occur inside the $k$-space region where the low-energy approximation that is widely used (namely, a modified Dirac equation) is valid and at points that are well within an estimated Fermi wavevector $k_{f}$ resulting from surface carriers.

In order to present our analysis in the jellium model, first it is useful to remind the reader of systems that are a little more traditional, in the sense of being well-studied, than the above. For example, the standard sawtooth behaviour of the low-temperature magnetization of an electron gas in 2D interfaces and in the presence of an external perpendicular magnetic field is well-known both from experimental measurements [15], as well as from analytical calculations of the total energy of a noninteracting electron system with the use of a picture of LLs in a canonical ensemble approach (reviewed in Section 2). This sawtooth behaviour occurs as a function of the magnetic field. As a function of the inverse field, the "saw" has periodic steps, signifying the appearance of (or actually defining) the standard $\mathrm{dHvA}$ effect. In this article, we go further than these calculations by taking the 
issue of nonzero thickness of the interface seriously and by making a systematic study of its role on the ground state energetics of the interface, also by commenting on transport properties. We present extensions of the above type of analytical calculations to a quasi-2D interface with a finite-thickness QW in the $z$-direction, parallel to the magnetic field, by using rigid boundary conditions at the two edges of the QW, i.e., with an infinite potential barrier to represent the vacuum - similar to the "open boundary conditions" used in the area of 3D topological insulators. We also present independent analytical calculations, which are extensions of those that have already been carried out earlier in systems of astrophysical interest, for a fully $3 \mathrm{D}$ quantum system of noninteracting electrons in infinite space and in an external magnetic field with periodic boundary conditions parallel to the field, all at zero temperature $(\mathrm{T}=0)$. Both systems, the quasi-2D interface and the full $3 \mathrm{D}$ space, seem to lead to previously unnoticed features in each system's magnetic response properties. For the interface, the crucial point is the single-particle energy competition between the LLs and the QW-levels for the different types of occupation-scenarios that are possible and allowed by the Pauli Exclusion Principle, when one attempts to determine the lowest total energy of the many-electron system. The basic physical reason is that each oneparticle state is now characterised by three quantum numbers. There are then cases when the system energetically prefers to change (increase) a $z$-mode and then it can, or in fact it must, go back to lower quantum numbers of the 2D motion (in our case LLs) without violating Pauli's principle and in so doing, it can acquire a lower (in fact the lowest possible) total energy. In this paper, it is shown that the manner in which occupancies (and transitions) occur, according to the above criteria, is an interesting and nontrivial exercise with the total energy probably not reducible to closed analytical forms immediately when an arbitrary field and an arbitrary thickness are given. One actually has to run the occupation scenarios starting from special values of parameters (for which the problem is easy) and then vary these parameters in some well-defined manner until they assume their values under consideration. When this exercise is carefully and properly solved, it defines a sequence of critical fields (or correspondingly of QW thicknesses) where "internal transitions" occur, in the sense that the highest LLs are only partially filled, which in turn leads to a number of new singular features in global magnetisation and in magnetic susceptibility. As a result, nontrivial quantal corrections to, or more appropriately, violations of the standard dHvA periodicities are found. In the independent calculation in full 3D infinite space, we determine the exact quantal behaviour of magnetisation, which in strong magnetic fields is found to deviate considerably from the standard semi-classical dHvA period but is also found to rapidly converge to this semi-classical periodicity as the magnetic field is reduced. The complete solution of this latter problem, derived here in closed form, also demonstrates some interesting analytical patterns in terms of the Hurwitz zeta functions that seem to have not been properly identified in earlier works. The mathematical problem of how to go analytically from the quasi-2D results to the results of the full 3D system (in the limit of infinite thickness) is also tackled; thus, providing a test and a proof of correctness and consistency of all the analytical expressions found here to describe the quasi-2D interface problem. Upon inclusion of Zeeman splitting, additional features are also highlighted, such as certain minima in total energy that originate from the interplay of QW, Zeeman and LL Physics in the full 3D problem, which might possibly be useful for the design of stable 3D quantum devices, i.e., in cases where the magnetic field can be self-consistently considered as self-generated. Furthermore, a corresponding calculation, now with the so-called $\Lambda$-Levels in place of LLs in a Composite Fermion picture, in the approximation of noninteracting Composite Fermions, demonstrates the utility of our method, because it leads to new predictions on magnetic response properties of a fully-interacting electron liquid, possessing a certain form of universality, in which the finite thickness of the interface plays a major role, albeit different from earlier works such as [11]. These predictions should be compared with the much earlier reported mere monotonic reduction of FQHE gaps with thickness (see [16] for conventional FQHE systems - while for recent topologically nontrivial systems see [17]). In our results, they exhibit a richer and more delicate structure that possibly could be detectable with present day technology.

In the bulk of this article, particles are assumed nonrelativistic with a parabolic spectrum. A similar procedure for a model system with the relativistic energy spectrum of Graphene in the plane could be easily followed, although this is something that is not pursued here. Moreover, the method of energy-interplays presented in this work is immediately extendable to include Rashba or other types of spin-orbit coupling [18,19], although we will not consider this either in the present article. However, towards the end of the article, we do provide hints of relevance or of the applicability of the present method to analogous systems, namely systems with topologically nontrivial $k$-space behaviours, such as the dimensionality crossover from a 3D to a $2 \mathrm{D}$ topological insulator, i.e., systems with strong spin-orbit coupling and with low-energy properties described by a Dirac-type of equation.

The paper is organised as follows: Section 2 briefly reviews the energetics and QHE transport properties of a 2D system of noninteracting electrons in a perpendicular magnetic field, by placing emphasis on the thermodynamic functions of the system and on how the dHvA periodicities directly come out, although a relevant discussion of transverse conductivity is also briefly made. Section 3 deals with the same system confined in an interface of nonzero thickness $d$ with no magnetic field applied, presenting a systematic study of the energy behaviour for several thicknesses. Even this seemingly trivial problem leads to interesting behaviours, such as a sequence of 
Fermi circles (or disks) associated with each QW-level that are generally different from the circular crosssections (of a 3D Fermi surface) that result from earlier semi-classical treatments through the intersection of $k_{z}$ with a predetermined Fermi sphere, reproducing those only when there is a large number of QW-levels involved. For small QW numbers, it is shown that when the thickness $d$ is below a critical value, the system can be considered as two dimensional, whereas for very large $d$ we recover the energy of 3D noninteracting electron gas. Moving forward, in Section 4 we apply on the interface a uniform perpendicular magnetic field $B$ and study in detail all the thermodynamic properties, such as energy, magnetisation and susceptibility for several values of $d$ and $B$, or under combined variations of both, demonstrating that they exhibit a rich pattern of behaviours in a rather unpredictable manner. [Transport properties are also discussed and they have a great resemblance to the corresponding $2 \mathrm{D}$ results, which is rather expected for such a conventional system, being essentially a multilayered QHE system.] An inclusion of Zeeman coupling modifies the results (they now depend strongly on the gyromagnetic ratio) and an inclusion of interactions in a Composite Fermion picture gives further, not easily predictable corrections and a type of universality. Section 5 presents the original electronic problem in full 3D space, where the electrons are confined in a large macroscopic cube with periodic boundary conditions along the field direction and we present exact analytical expressions of all thermodynamic properties, using a method not usually applied to solid-state systems but more often associated with astrophysical treatments. We find in this problem a sequence of Fermi lines (segments) associated with each LL, which again are generally different from results of semi-classical treatments determined by semi-classical Landau tubes inside (and intersecting) a predetermined Fermi sphere, reproducing those only when there is a large number of LLs involved. However, what is more gratifying is that the results are shown analytically to be consistent with the limiting behaviour of the corresponding results of the quasi-2D interface when its thickness goes to infinity; the fine details of the quasi-2D calculation being essential for reproducing this limit. We also recover for the full 3D problem the dHvA periodicities in the limit of weak $B$, while for large $B$ s we provide the exact quantal violations of (or deviations from) these semi-classical periodicities. We also give estimates of particle densities for which such violations might be detectable in 3D solid-state systems. Finally, in Section 6 we turn our attention to the applicability of our method to the more interesting problem of the dimensionality crossover from a 3D topological insulator, possessing a single Dirac cone on its surface, to a 2D topological insulator quantum well. It is demonstrated briefly, how this line of reasoning could be pursued, even in this case where the thicknessrelated modes are strongly coupled to the planar degrees of freedom and it is argued that the effects of the above type might also be present in these more exotic systems. Section 7 summarises our conclusions.

\section{Nonrelativistic electron gas in $2 \mathrm{D}$ in a perpendicular magnetic field}

As a precursor to the main results of this work, we begin with the well-known problem of a system of many $(N)$ noninteracting electrons, each with charge $-e$, effective mass $m$ and spin $s$ that are free to move in a $2 \mathrm{D}$ plane in the presence of an external homogeneous magnetic field $B$ perpendicular to the plane, at temperature $\mathrm{T}=0$. For simplicity, let us first ignore the Zeeman splitting, i.e., we take the gyromagnetic ratio $\mathrm{g}^{*}=0-$ however, note that we consider particles that $d o$ have spin (i.e., $s=1 / 2$ ); thus, providing a slightly more complete treatment than the standard (academic) one with spinless fermions. As is well known, this simple jellium model accounts for both the thermodynamic and transport properties of electrons, as these are observed in experiments on QHE systems in properties, such as magnetisation or Hall magnetoresistivities. However, we should state at the outset, that although these types of systems (interfaces or films) are not purely $2 \mathrm{D}$, we can always reduce their thickness to achieve an effectively two-dimensional system (see Section 3 for the corresponding "critical thickness", which depends on the areal density of electrons, as this is rigorously determined (at $\mathrm{T}=0$ ) by our analytical calculations).

It is well known that in this $2 \mathrm{D}$ problem, the orbital motion of noninteracting electrons, which satisfy the nonrelativistic Schrodinger equation, is described by a Landau Level (LL) picture for the single-particle energy spectrum, namely

$$
\varepsilon_{n}=\left(n+\frac{1}{2}\right) \hbar \omega_{c},
$$

where $\omega_{c}=e B / m c$ is the cyclotron frequency, $e$ is the absolute value of charge of each electron and $n$ (the LL index) is a non-negative integer that characterises all LLs. It is also well known that each LL has degeneracy $2 \Phi / \Phi_{0}$ (accounting for the spin $s=(1 / 2)$ of each electron - more generally, the prefactor being $\left.2 s+1\right)$, where $\Phi$ is the total magnetic flux passing through the system and $\Phi_{0}$ is the flux quantum $\left(\Phi_{0}=h c / e\right)$. Each LL can then 
contain $2 \Phi / \Phi_{0}$ electrons (due to Pauli's principle at $\mathrm{T}=0$ ) such that in the most general case, when there are $\rho(\mathrm{a}$ positive integer) LLs occupied by electrons (namely $\rho=n+1$, with $n$ the LL index of the highest occupied level) the following inequality is satisfied

$$
2(\rho-1) \frac{\Phi}{\Phi_{o}} \leq N \leq 2 \rho \frac{\Phi}{\Phi_{o}}
$$

or equivalently (given that $\Phi=B S$, with $S$ being the total surface area of the sample)

$$
\frac{1}{2(\rho-1)} n_{A} \Phi_{o} \geq B \geq \frac{1}{2 \rho} n_{A} \Phi_{o},
$$

where $N$ is the total number of particles and $n_{A}=N / S$ is their areal density. (Note that we follow a picture of a constant number of electrons (canonical ensemble), although this does not hurt generality as we will see later.) When the magnetic field varies in the above window, the electrons occupy $\rho$ LLs (where the last occupied level of LL index $\rho-1$ is not necessarily completely filled up; a complete filling merely corresponds to an equality in the right-hand side of (2.3)). First, if $\rho=1$, valid for $B \geq 1 / 2 n_{A} \Phi_{o}$, all electrons are accommodated in the lowest LL and the total energy is simply

$$
E=N \frac{\hbar \omega_{c}}{2}
$$

and it is therefore linear in $B$. For many LLs $(\rho>1)$, it is easy to sum over all occupied LLs to find the total energy of the system, namely

$$
E=2 \frac{\Phi}{\Phi_{o}} \sum_{n=0}^{\rho-2}\left[\hbar \omega_{c}(n+1 / 2)\right]+\left(N-2(\rho-1) \frac{\Phi}{\Phi_{o}}\right) \hbar \omega_{c}(\rho-1+1 / 2) .
$$

Then by using the sums

$$
\begin{aligned}
\sum_{n=0}^{\rho-2} n & =\frac{(\rho-1)^{2}-(\rho-1)}{2} \\
\text { and } \quad & \sum_{n=0}^{\rho-2} \frac{1}{2}=\frac{(\rho-1)}{2},
\end{aligned}
$$

we can determine the total energy in units of 2D Fermi energy $\left(E_{f}=\frac{\hbar^{2} k_{f}^{2}}{2 m}, k_{f}=\sqrt{2 \pi n_{A}}\right)$, which has the following final form:

$$
E=N E_{f}\left[2\left(-\rho^{2}+\rho\right)\left(\frac{B}{n_{A} \Phi_{o}}\right)^{2}+2\left(\frac{B}{n_{A} \Phi_{o}}\right)\left(\rho-\frac{1}{2}\right)\right]
$$

One immediately notes that the energy varies quadratically with respect to $B$ (for $\rho>1$ ), such that one notes a linear behaviour of the magnetisation or a constant value of the magnetic susceptibility, quantities that are determined by derivatives of $E$ with respect to $B$, as discussed further below. As already mentioned, for very strong $B$, i.e., for $B \geq 1 / 2 n_{A} \Phi_{o}$ (such that $\rho=1$ ) $E$ is given only by the last term in (2.7) and is linear in $B$, the magnetisation being therefore constant and having the value $-N \mu_{B}$ with $\mu_{B}$ the Bohr magneton, an "atomic value" of magnetic moment that is expected for almost nonoverlapping particles in the strong field limit (see more general discussion below).

From application of the first law of thermodynamics at $\mathrm{T}=0$ one can determine the global magnetisation $M$ (it is actually the total magnetic moment of the system, i.e., an extensive quantity but here, we will follow the usual terminology) and magnetic susceptibility $\chi$ through simple derivatives of (2.7), namely 


$$
M=-\left.\frac{\partial E}{\partial B}\right|_{V} \text { and } \chi=\left.\frac{\partial M}{\partial B}\right|_{V}
$$

and these turn out to give

$$
\begin{gathered}
M=N \frac{E_{f}}{\left(n_{A} \Phi_{o}\right)}\left[4\left(\rho^{2}-\rho\right) \frac{B}{\left(n_{A} \Phi_{o}\right)}-2\left(\rho-\frac{1}{2}\right)\right] \\
\chi=N \frac{E_{f}}{\left(n_{A} \Phi_{o}\right)^{2}}\left[4\left(\rho^{2}-\rho\right)\right] .
\end{gathered}
$$

It should be noted that $\chi$ is always non-negative for this $2 \mathrm{D}$ case; it is probably useful to state early on that when we later include a thickness for our interface, we will find cases (ranges of parameters) where $\chi$ will also assume negative values. If the magnetisation is measured in units of Bohr magneton $\left(\mu_{B}=e \hbar / 2 m c=E_{f} / n_{A} \Phi_{o}\right)$, etc., the above results are represented by the figures shown below.

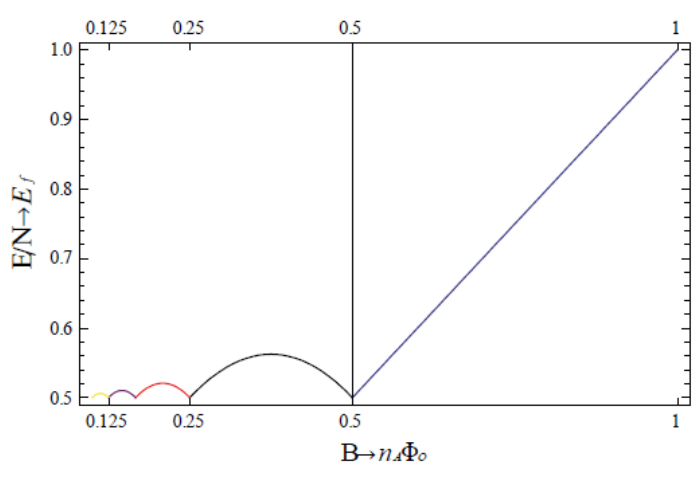

FIG. 2.1: Energy per electron (in units of 2D Fermi energy)

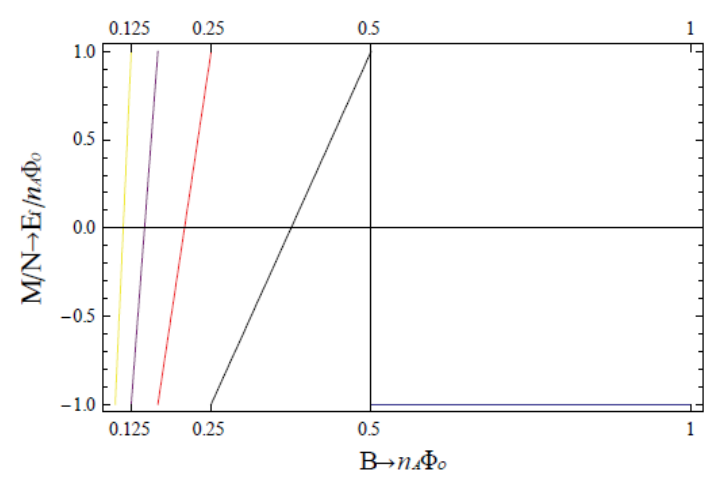

FIG. 2.2: Magnetisation per electron (in units of $\mu_{B}$ )

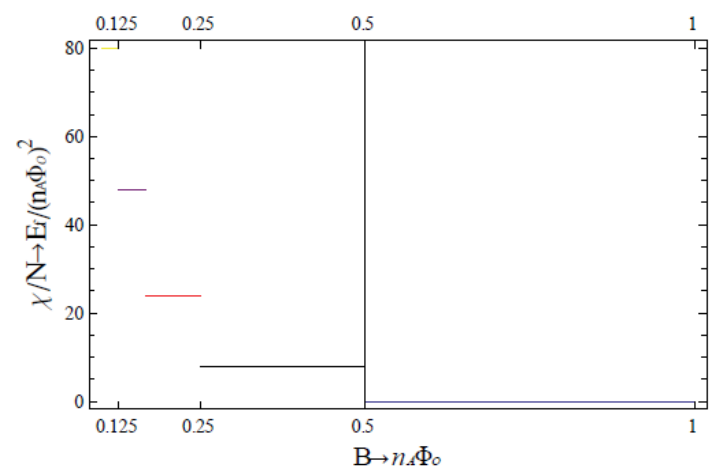

FIG. 2.3: Susceptibility per electron (in units of $\mu_{B} / n_{A} \Phi_{0}$ )

Therefore, in this manner, one obtains the well-known sharp sawtooth behaviour of magnetisation (in a system with a constant number of electrons) measured in low-T experiments [15]. If the above were plotted as a function of $1 / B$, then the above windows would be periodically repeated with a period $\Delta(1 / \mathrm{B})=2 / n_{A} \Phi_{o}$, which is compatible with the dHvA period $2 \pi e / \hbar c A_{f}$ (with $A_{f}=\pi k_{f}^{2}$ and $k_{f}^{2}=2 \pi n_{A}$ ) (see, e.g., [6]). Also note that, for $B \rightarrow 0$, the above energy correctly reproduces the $2 \mathrm{D}$ noninteracting result $E / N=1 / 2 E_{f}$ (i.e., in (2.7) take $B \rightarrow 0$ and $\rho \rightarrow \infty$ in such a way that the product $B \rho$ is fixed). 


\section{Relation to transport properties - Hall conductivity}

It is useful to mention in passing a physical interpretation of the above thermodynamic results (at $\mathrm{T}=0$ ), which has a connection to transport properties and that in particular relates the above magnetisation discontinuities with diamagnetic currents. Indeed, the discontinuities in $M$ can be associated with the abrupt change of chiral currents on the edges, despite the fact that the edges did not directly enter anywhere in the above formulation. This connection is through the simple relation of the magnetisation $M$ with the diamagnetic electric currents $I$ that flow around the edges (in opposite directions), namely $M=I S / c$ (as one can immediately see by comparing $M=-\partial E / \partial B$ with the Aharonov-Bohm formula $I=-c \partial E / \partial \Phi$, if $I$ is assumed flowing along the edges such that the flux $\Phi=B S$ can be viewed as an enclosed flux), in combination with the quantised values of the Hall conductance ( $\sigma_{\mathrm{H}}=2 \rho e^{2} / h$ for spinfull electrons) and the fact that, during the transitions to a different LL, the current responds to a transverse potential that is equal to $\Delta \mu=\hbar \omega_{c}$ divided by $e$. Therefore, we expect to have (for the magnitudes of the various quantities involved)

$$
I=\sigma_{\mathrm{H}} \frac{\Delta \mu}{e} \text { with } \Delta \mu=\hbar \omega_{c} \quad \rightarrow \quad \Delta \mathrm{M}=\sigma_{\mathrm{H}} \frac{\Delta \mu S}{e c}=2 N \mu_{B}
$$

where in the above, the values of $B=n_{A} \Phi_{o} / 2 \rho$ (where the transitions occur) have been used in the last step; therefore (2.10) gives the correct magnitude of discontinuities $2 N \mu_{B}$ for the magnetisation that we see in Fig.2.2, which occur whenever we have complete filling of $\rho$ LLs. [For completeness, we simply mention here that the above could have also been derived with the well-known Widom-Streda formula combined with a thermodynamic Maxwell relation, a more frequently followed procedure that gives $\Delta \mathrm{M}=N \Delta \mu / B$ (for the simultaneous discontinuities of $M$ and $\mu$ ), which turns out to be equivalent to (2.10) but the above given diamagnetic current interpretation is preferable if we want to later generalise in a similar line of reasoning to the finite-thickness case (see corresponding discussion of transport in Section 4).]

The above also shows immediately how the discontinuities of $M$ are directly related to the Hall conductance. One could determine $\sigma_{\mathrm{H}}$ from (2.10) by measuring the simultaneous discontinuities of $M$ and $\mu$. This is a line that is actually going to be followed in the finite-thickness case of Section 4.

The electron gas in full 3D space inside a homogeneous magnetic field would normally be the next example to consider and it will indeed be discussed in Section 5. This problem has mostly been treated in astrophysical applications but here, we want to place it within a framework interesting to fully 3D solid-state systems. Although it might be useful to present it at this point, in order to see the rather large differences from the above 2D case, e.g., the smooth deviations from the above dHvA periods, we choose to present it after discussion of the quasi-2D cases that follow. In this manner, we can study in detail the dimensionality crossover from $2 \mathrm{D}$ to $3 \mathrm{D}$, thus, addressing issues regarding possible $\mathrm{dHvA}$ violations both in quasi-2D and in bulk $3 \mathrm{D}$ solids in a unifying manner.

\section{Finite-thickness interface (without magnetic field)}

Let us now consider an interface with a finite (nonzero and non-infinite) thickness $d$ but let us first begin with the simpler problem of a vanishing magnetic field. In this case, we will see that the standard Fermi circle or disk of 2D noninteracting electrons in the jellium model will now be replaced by a sequence of many Fermi circles of appropriate radii, each one connected to a particular QW-level associated with the $z$-motion; the procedure of determining the appropriate radii being not so trivial and rather tedious, as we shall see. Once again, we will work in the canonical ensemble with a fixed number $\mathrm{N}$ of electrons, such that the surface areal density $n_{A}$ is the control parameter, although at the end this can be relaxed. The results can recover those that would have been obtained if the control parameter were the volume density $n_{V}=n_{A} / d$ (see later below) and especially so, in the limit $d \rightarrow \infty$.

Indeed, consider an interface (or film) that again extends in a macroscopically large area $S$ in the $x$ and $y$ directions, whereas in the $z$-direction it is characterised by a width $d$, which we can initially consider as very small (of the order of nanometers, i.e., a few atomic layers thick). In the jellium model that we consider here, the Hamiltonian is effectively just a nonrelativistic kinetic energy term in 3D space, namely 


$$
\frac{P^{2}}{2 m} \Psi=E \Psi
$$

with $\vec{P}$ being the canonical momentum (we have obviously taken the simplest gauge $\vec{A}=0$ ). For a large system on a plane, it is natural to impose periodic boundary conditions in the $x$ and $y$ directions but the $z$ axis can be treated like a 1D double quantum well with impenetrable walls at $z=0$ and $z=d$ (the simplest way to impose the spatial confinement). The eigenfunctions of (3.1) can then be written as simple product functions of the form

$$
\Psi(x, y, z) \propto \sin \left(k_{z} z\right) e^{i k_{y} y} e^{i k x x} .
$$

A quantum state is then characterised by the eigenvalues of the three Cartesian components of canonical momentum $\vec{P} / \hbar\left\{k_{x}, k_{y}, k_{z}\right\}$ (or $\left\{n_{x}, n_{y}, n_{z}\right\}$ after quantisation, see below). The Pauli principle requires that each such orbital state (namely a triplet $\left\{n_{x}, n_{y}, n_{z}\right\}$ ) can be occupied at $\mathrm{T}=0$ by only two electrons (of opposite spins) and this is a very important criterion, which for strongly anisotropic systems such as this one, must be imposed in a careful manner, as we will see below and also in later Sections. The single-particle energy spectrum is

$$
\begin{gathered}
\mathcal{E}_{n x, n_{y}, n_{z}}=\frac{\hbar^{2} k^{2}}{2 m}+\mathcal{E}_{n_{z}} \text { where } \boldsymbol{\varepsilon}_{n z}=\frac{\hbar^{2} k_{z}{ }^{2}}{2 m} \text { and } k^{2}=k_{x}{ }^{2}+k_{y}{ }^{2}, k_{x}=2 \pi \frac{n_{x}}{L_{x}}, k_{y}=2 \pi \frac{n_{y}}{L_{y}}, k_{z}=\pi \frac{n_{z}}{d} \\
\left(n_{x}, n_{y}\right)=(0, \pm 1, \pm 2 \ldots), n_{z}=1,2,3 \ldots
\end{gathered}
$$

i.e., $k_{x}$ and $k_{y}$ are quasicontinuous variables (because $L_{x}, L_{y} \rightarrow \infty$ ), whereas $k_{z}$ is strongly quantised. For extremely small $d$, the variable $k_{z}$ is expected to take its lowest value (corresponding to $n_{z}=1$ ) for all electrons, which is the case that is usually discussed in the literature, where the particles are "frozen" at the lowest QW-level $n_{z}=1$, making the system effectively 2D. This is so, because of the enormous energy difference between the $n_{z}=2$ and $n_{z}=1$ levels (that goes as $1 / d^{2}$ ) and therefore, because it is indeed energetically favourable to start filling states with increasing $\left|k_{x}\right|$ and $\left|k_{y}\right|$ (or equivalently $\left|n_{x}\right|$ and $\left|n_{y}\right|$, starting from 0 and gradually occupying higher numbers in a symmetric manner, with $n_{z}$ always being 1), thus, forming the standard Fermi circle of $2 \mathrm{D}$ noninteracting electrons. However, the reader should note that for any fixed nonzero $d$, even at $\mathrm{T}=0$, the above-mentioned $2 \mathrm{D}$ character may be violated for sufficiently large density (to be quantified below). There might come a point (i.e., if the number of electrons to be accommodated in single-particle states is sufficiently large) when it is no longer favourable to continue increasing the Fermi circle and maintain $n_{z}=1$; it may be favourable for the remaining electrons to start jumping to the $n_{z}=2$ QW-level and then $k_{x}$ and $k_{y}$ can start taking values back at $\left|n_{x}\right|=\left|n_{y}\right|=0$, i.e., start forming a new Fermi circle, now associated with the level $n_{z}=2$. We emphasise that this occurs without violating Pauli's principle, because in the triplet $\left\{n_{x}, n_{y}, n_{z}\right\}$, which labels a single-particle state, $n_{z}$ has changed value, so that $n_{x}$ and $n_{y}$ can now acquire the same values as they had before this transition, starting again from 0 . The transition to $n_{z}=2$ will of course occur whenever the "initial" Fermi circle (for $n_{z}=1$ ) becomes so large (with such a long radius $k_{f 1}$ ) that the single-particle energy $\hbar^{2} k_{f 1}^{2} / 2 m$ will become equal with and from that point on, exceed the energy difference between the two QW-levels, or equivalently, whenever the following equality holds

$$
\frac{\hbar^{2} k_{f 1}^{2}}{2 m}+\varepsilon_{n z=1}=\varepsilon_{n z=2}
$$

The left-hand-side of (3.4) is the single-particle energy of an "extra" electron that we wish to place on the perimeter of the Fermi circle, previously formed by all other electrons that were in the QW-level $n_{z}=1$. The right-hand-side of (3.4) is the analogous single-particle energy if we were to put the "extra" electron at the QWlevel $n_{z}=2$ and start a new Fermi circle from the beginning, namely from zero radius.

It is now important to note that (3.4) provides a sense of "equilibrium" in the occupation procedure. As stated, from that point on, a $2^{\text {nd }}$ Fermi circle is being formed (corresponding to $n_{z}=2$ ) and what is more important, the above sense of "equilibrium" must be preserved during the entire occupation procedure that follows. If we still have an excess of electrons and we keep occupying available (empty) single-particle states, then the extra electrons must be placed back and forth in both QW-levels $n_{z}=1$ and $n_{z}=2$ in a way that the Fermi radius associated with $n_{z}=1$ and the one associated with $n_{z}=2$ will both keep increasing and will at every point (for every density) be related with each other through the "equilibrium" relation 


$$
\frac{\hbar^{2} k_{f 1}^{2}}{2 m}+\varepsilon_{n z=1}=\frac{\hbar^{2} k_{f 2}^{2}}{2 m}+\varepsilon_{n z=2}
$$

such that the occupation procedure is "fair", guaranteeing that it will give the lowest possible total energy for the many-particle system. (3.5) demands that the extra electron to be placed anywhere at any moment of the occupation procedure must have the same single-particle energy in any of the possible occupational scenarios. [For the same line of reasoning as this applied to different problems, see also (4.3) and (5.7)]. If the equality of (3.5) were not satisfied and one side were larger than the other, it would mean that the procedure followed up to that point was not the optimal (energetically lowest) one, because we could always move electrons around in state-space to gain energy. [It can actually be shown variationally [20] that the above procedure is the lowest energetically.] The reader should notice that this "fairness" strategy is actually a generalisation of the standard symmetric manner of occupation scenarios that are followed in the usual construction of the 3D Fermi surface, where this "equilibrium" in the single-particle states occupation procedure is the usual isotropic filling in $k$-space that leads to the standard Fermi sphere; the above is a generalisation of this to a highly anisotropic system. This optimal partitioning for our anisotropic problem (in the above-described cases of one or two Fermi circles) is represented pictorially in Fig.3.1 and Fig.3.2.

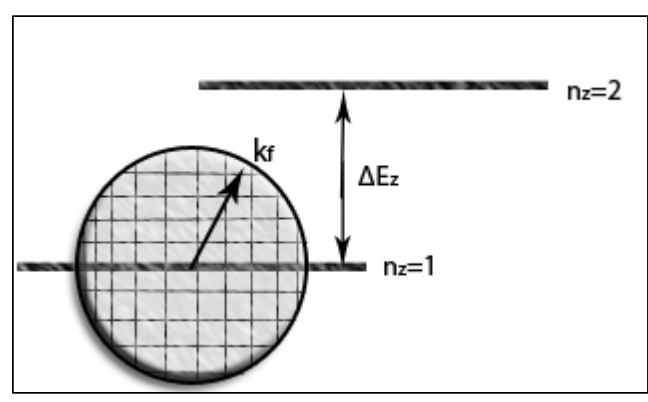

FIG. 3.1: Only one Fermi circle is created $(p=1)$ when $d<d_{\text {crit } 1}$

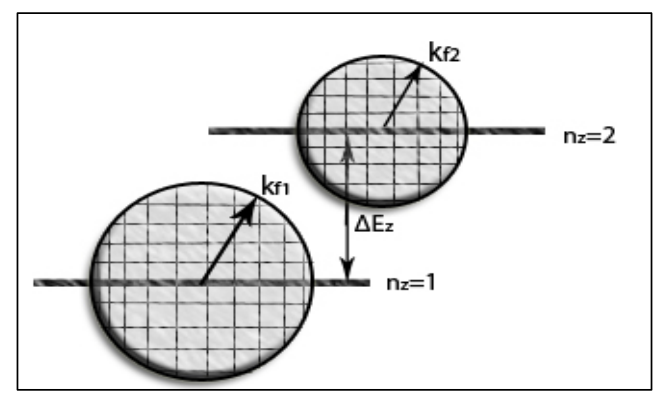

FIG. 3.2: Two Fermi circles are created $(p=2)$ when $d_{\text {crit } 1}<d<d_{\text {crit } 2}$

In the (usual) case of only one QW-level being enough to accommodate all particles (Fig.3.1), it transpires from (3.4) that $d$ must be

$$
d<d_{\text {crit } 1}=\sqrt{\frac{3 \pi}{2 n_{A}}}
$$

that gives a rigorous quantitative measure of what is meant by two-dimensionality and in such a case of sufficiently small $d$, the total energy per electron is simply

$$
\frac{E}{N}=E_{f}\left(\frac{1}{2}+\frac{\pi}{2 n_{A} d^{2}}\right) \text {, }
$$

the usual 2D result plus a constant term (note that $E_{f}$ is always the 2D Fermi energy $\left(E_{f}=\frac{\hbar^{2} k_{f}^{2}}{2 m}, k_{f}=\sqrt{2 \pi n_{A}}\right)$ ). As seen above, $d_{c r i t 1}$ depends on $n_{A}$. For $n_{A}=10^{16} \mathrm{~m}^{-2}$ the critical thickness is $21.7 \mathrm{~nm}$.

Alternatively, of course, the result is that for any given fixed $d$, there is a critical areal density

$$
n_{A_{c r i t}}=\frac{3 \pi}{2 d^{2}}
$$

below which (i.e., for $n_{A}<n_{A}$ crit ) the interface essentially behaves as 2D, having again the energy (3.7).

[The reader should note that if the volume density $n_{V}$ were the good variable, dividing both sides of (3.4) by $d$ would instead give the result $d_{c r i t 1}=\left(3 \pi / 2 n_{V}\right)^{1 / 3}$ as the criterion for two-dimensionality. This might be more appropriate for systems with a constant volume density (as $d$ changes) [21] rather than constant particle number, or for systems that anticipate a 3D Fermi surface in some semi-classical approximation [22,23]. However, here, 
we follow a more appropriate procedure and at the end, we will recover the previous results in the appropriate limit.]

In the case of two (and only two) QW-levels being necessary to accommodate all electrons (Fig.3.2), it transpires by solving (3.5) with respect to $k_{f 1}, k_{f 2}$ with the extra condition $n_{A}=n_{A_{1}}+n_{A_{2}}$ and the use of (3.13), that the optimal partition in the two Fermi circles is described by the (partial) areal densities

$$
\begin{aligned}
& n_{A_{1}}=\frac{n_{A}}{2}+\frac{3 \pi}{4 d^{2}}=\frac{n_{A}}{2}+\frac{n_{A_{c r i t}}}{2} \\
& n_{A_{2}}=\frac{n_{A}}{2}-\frac{3 \pi}{4 d^{2}}=\frac{n_{A}}{2}-\frac{n_{A_{c r i t}}}{2}
\end{aligned}
$$

and that this occurs whenever $d_{c r i t 1}=\sqrt{3 \pi / 2 n_{A}}<d<d_{\text {crit } 2}=\sqrt{13 \pi / 2 n_{A}}$, with $d_{c r i t 2}$ being determined by another equilibrium condition analogous to (3.4), namely

$$
\frac{\hbar^{2} k_{f 1}^{2}}{2 m}+\varepsilon_{n z=1}=\varepsilon_{n z=3}, \quad \text { or } \quad \frac{\hbar^{2} k_{f 1}^{2}}{2 m}=\frac{8 \hbar^{2} \pi^{2}}{2 m d^{2}}
$$

together with (3.13) and in combination with

$$
n_{A}=n_{A_{1}}+n_{A_{2}}
$$

Finally, in the above case (of Fig.3.2), the total energy that has contribution from two QW-levels and two Fermi circles turns out to be

$$
\frac{E}{N}=E_{f}\left(\frac{1}{4}+\frac{5 \pi}{4 n_{A} d^{2}}-\frac{9 \pi^{2}}{16 n_{A}^{2} d^{4}}\right)
$$

(valid when $d_{\text {crit } 1}<d<d_{\text {crit } 2}$ ). After having given the main physical idea with the above examples, let us in the following solve the problem in full generality, i.e., generalise this line of reasoning to any arbitrary number of QW-levels playing a role in the energy partition. In the sense discussed above, to each quantum number $n_{z}=1,2 \ldots$ there corresponds a different 2D Fermi circle of radius $k f_{n z}$ and an associated areal density of electrons $n_{n z}$ that satisfy

$$
k_{f_{z z}}=\sqrt{2 \pi n_{A z}}
$$

as is easy to show with a standard 2D argument for spinfull electrons. The immediate question is how to determine in the most general case the proper (i.e., lowest-total-energy) partition of the total number (or density) of electrons to the correct values of $n_{A_{1}}, n_{A_{2}}$, etc., which are generally many; their actual number depending of course, on the value of thickness $d$. As $d$ becomes exceedingly large, we expect more and more QW levels to play a role and in such a case, we expect the results of the above procedure to tend towards the previous semiclassical results with the relevant variable being the volume density $n_{V}$ [22,23]. Indeed, for a check and for exactly how we recover the correct limit, see (3.30).

The technique to determine the correct partition in the general case is rather simple (and it was already motivated for two QW-levels). At every point we must have "equilibrium" in the sense discussed above but now for many (an arbitrary number of) $z$-levels. Let us suppose that the width $d$ of the interface is such that all electrons occupy $p z$-axis levels (generalising the earlier examples that would correspond to $p=1$ and $p=2$ ). This means that there is a total of $p$ Fermi circles created in the system, each circle labelled by a particular value of the quantum number $n_{z}$. Now, for a given (constant) value $d$ of thickness, the single-particle energy of an extra electron that we wish to place at the perimeter of a Fermi circle (of a particular $n_{z}$ ) must be equal to the corresponding singleparticle energy of the same electron, if it were placed at the perimeter of any other Fermi circle (for different $n_{z} \mathrm{~s}$ ); this being a reflection of the "equilibrium" noted above, which guarantees the lowest total energy, namely 


$$
\frac{\hbar^{2} k_{f 1}^{2}}{2 m}+\frac{\hbar^{2} \pi^{2}}{2 m d^{2}}=\frac{\hbar^{2} k_{f 2}^{2}}{2 m}+\frac{4 \hbar^{2} \pi^{2}}{2 m d^{2}}=\ldots=\frac{\hbar^{2} k_{f p}^{2}}{2 m}+\frac{p^{2} \hbar^{2} \pi^{2}}{2 m d^{2}}
$$

Now, using the above relations (viewed as a system of $p$ - 1 equations for the $k_{f}$ 's), we can determine all the partial areal densities, corresponding to each $n_{z}$, as functions of the density of the electrons that belong to $n_{z}=1$. By solving the above system of equations, we find the optimal partition to be described compactly by

$$
n_{A j}=n_{A 1}-\frac{\left(j^{2}-1\right) \pi}{2 d^{2}}, \text { where the index } j \text { runs from } 1 \text { to } p \text {. }
$$

However, of course, the total sum of all partial densities must give the total areal density of the system

$$
n_{A}=\sum_{j=1}^{p} n_{A j}
$$

Using (3.15) and (3.16), we determine the areal density corresponding to $n_{z}=1$ analytically, the result being

$$
n_{A 1}=\frac{n_{A}}{p}+\frac{\pi}{2 d^{2}}\left[\frac{1}{p} \sum_{j=1}^{p} j^{2}-1\right]=\frac{n_{A}}{p}+\frac{\pi}{2 d^{2}}\left[\frac{1}{6}(p+1)(2 p+1)-1\right] .
$$

Substituting (3.17) into (3.15), we can find all partial densities (in the energetically optimal configuration and hence, the ground state of the many-electron system) in closed form and all results can be finally expressed by

$$
n_{A j}=\frac{n_{A}}{p}+\frac{\pi}{12 d^{2}}(p+1)(2 p+1)-\frac{j^{2} \pi}{2 d^{2}}
$$

with $j=1, \ldots, p$. Notice that for $p=1$ and $j=1$, we obtain $n_{A 1}=n_{A}$, i.e., all electrons occupy only the lowest QWlevel, as assumed, whereas for $p=2, j=1$ and $j=2$, (3.18) reproduces both of (3.9); observations that can be viewed as consistency tests. However, we have not yet retrieved the most useful information and in addition, it is useful practically to calculate the range of values of thickness necessary for the system to actually occupy exactly the above assumed $p$ levels of the QW. However, this is not difficult to determine; it becomes necessary to start to use all the $p \mathrm{QW}$-states whenever the $p^{\text {th }}$ Fermi circle is just about to form. The equilibrium condition then requires that (assuming $p>1$ )

$$
\frac{\hbar^{2} k_{f 1}^{2}}{2 m}+\frac{\hbar^{2} \pi^{2}}{2 m d^{2}}=\frac{p^{2} \hbar^{2} \pi^{2}}{2 m d^{2}}
$$

By solving this equation with respect to the sample thickness $d$ and by using (3.13) and (3.17), we find a series of critical values of thickness (for various values of $p=1,2,3, \ldots$ ), namely

$$
d_{c r i t}(p)=\sqrt{\frac{p(p-1)(4 p+1) \pi}{12 n_{A}}} .
$$

That is, for values of thickness larger than (3.20), the system occupies $p$ QW-levels, until the ( $p+1)$ Fermi circle starts over. This of course happens (as can be seen by just replacing $p$ with $p+1$ in (3.20)) when $d$ is equal to

$$
d_{c r i t}(p+1)=\sqrt{\frac{p(p+1)(4 p+5) \pi}{12 n_{A}}}
$$

Therefore, the conclusion is that when the thickness $d$ varies in the following window

$$
d_{c r i t}(p) \leq d \leq d_{c r i t}(p+1)
$$

the system occupies $p$ (and no more than $p$ ) QW-levels. 
The above results (3.20), (3.21) and (3.22) reproduce the previous examples for $p=1$ and 2: For

$$
0<d<d_{\text {crit }}(1)=\sqrt{3 \pi / 2 n_{A}} \quad(p=1),
$$

we have the case of Fig.3.1 (and the above (3.23) can be viewed as a criterion of 2-dimensionality). For

$$
d_{c r i t}(1)=\sqrt{3 \pi / 2 n_{A}}<d<d_{c r i t}(2)=\sqrt{13 \pi / 2 n_{A}} \quad(p=2),
$$

we have the case of Fig.3.2, where two Fermi circles are present, etc.

The final step is to determine in full generality, the total internal energy of the electron gas when $d$ lies in the range (3.22). This is given by

$$
E=\sum_{j=1}^{p}\left[\frac{1}{2} N_{j} E_{f j}+N_{j} \frac{j^{2} \hbar^{2} \pi^{2}}{2 m d^{2}}\right]
$$

with $N_{j}=n_{A j} S$ the number of electrons at QW-level $\mathrm{j}$ and hence, with $n_{A j}$ given by (3.18) and with $E_{f j}=\hbar^{2} k_{f j}^{2} / 2 m=\hbar^{2} 2 \pi n_{A j} / 2 m$, the corresponding 2D Fermi energy. Therefore, we have

$$
E=\frac{\hbar^{2}}{2 m} \sum_{j=1}^{p}\left[\pi n_{A j}{ }^{2} S+n_{A j} \frac{j^{2} \pi^{2}}{d^{2}} S\right]
$$

which after carrying out the sums, turns out to be

$$
E / N=\frac{\hbar^{2}\left(2 \pi n_{A}\right)}{2 m}\left[\frac{1}{2 p}+\frac{\pi(p+1)(2 p+1)}{12 n A d^{2}}-\frac{\pi^{2} p\left(p^{2}-1\right)(2 p+1)(8 p+11)}{1440 n^{2} d^{4}}\right]
$$

that gives directly the total ground state energy per electron, when the thickness of the system lies between (3.20) and (3.21). This reproduces the earlier results (3.7) for $p=1$ and (3.12) for $p=2$. We note that, even in this rather trivial system, the role of thickness on the energetics is noteworthy.

Once again we should stress that, compared with earlier work [21-23], (3.27) is exact and does not generally describe quantum oscillations with wavelengths that are governed by the extremal diameters of cross sections with an anticipated 3D Fermi surface; those being expected only for a large number of QW states, i.e., with very large $p$ 's involved. In contradistinction to earlier work, (3.27) is also valid for any small value of $p$.

A final point that is of interest is to take the thickness of our system to infinity but keeping the volume density $n_{V}=n_{A} / d$ constant. One expects that in the limit of infinite space, the above expression will converge to the well-known energy of noninteracting electrons in full 3D space, which is the standard result that is achieved through use of a macroscopically large cube. However, this has rather to be checked, because the standard problem that leads to the symmetric spherical Fermi surface utilises periodic boundary conditions in all Cartesian directions, whereas here we have infinite potentials (rigid boundary conditions) at two points of the $z$-axis. To examine if the above expectation is true, we choose to write the total energy in units of the 3D Fermi energy:

$$
E_{f}(3 D)=\frac{\hbar^{2}\left(3 \pi^{2} n_{V}\right)^{2 / 3}}{2 m} .
$$

We then have from (3.27)

$$
E / N=E f(3 D)\left(\frac{8}{9 \pi}\right)^{1 / 3}\left[\frac{n_{V}^{1 / 3} d}{2 p}+\frac{\pi(p+1)(2 p+1)}{12 n_{V}^{2 / 3} d^{2}}-\frac{\pi^{2} p\left(p^{2}-1\right)(2 p+1)(8 p+11)}{1440 n_{V}^{5 / 3} d^{5}}\right] .
$$


Substituting (3.20) into (3.28) we can plot the energy for large values of $p$, keeping the volume density $n_{V}$ constant (see Fig.3.3), from which it is readily seen that it indeed tends to the well-known energy of free electrons in full 3D space, namely

$$
E / N=\frac{3}{5} E f(3 D) \text {. }
$$

To see this analytically, we need to make explicit use of the volume density. From (3.20) and (3.21), after setting $n_{A}=n_{V} d$ and solving for $d$, we take the limits $d \rightarrow \infty$ and $p \rightarrow \infty$ such that the $d$ window of values is shrunk to only a single value

$$
d^{3}=\frac{p^{3} \pi}{3 n_{V}}
$$

and from (3.28) we then have

$$
E / N=E f(3 D)\left(\frac{8}{9 \pi}\right)^{1 / 3}\left[\frac{n_{V}^{1 / 3} d}{2 p}+\frac{\pi p^{2}}{6 n_{V}^{2}{ }^{2 / 3} d^{2}}-\frac{\pi^{2} p^{5}}{90 n_{V}^{5 / 3} d^{5}}\right] .
$$

Substituting $(d / p)^{3}=\pi / 3 n_{V}$ (due to (3.29)), we finally have

$$
E / N=E f(3 D)\left(\frac{8}{9 \pi}\right)^{1 / 3}\left[\frac{n_{V}^{1 / 3}}{2}\left(\frac{\pi}{3 n_{V}}\right)^{1 / 3}+\frac{\pi}{6 n_{V}^{2}}{ }^{2 / 3}\left(\frac{3 n_{V}}{\pi}\right)^{2 / 3}-\frac{\pi^{2}}{90 n_{V}^{5 / 3}}\left(\frac{3 n_{V}}{\pi}\right)^{5 / 3}\right],
$$

which transpires to be

$$
=\frac{3}{5} E f(3 D) .
$$

Therefore, we have given a full analytical treatment of the dimensionality crossover of nonrelativistic noninteracting electrons (in zero-field) from pure 2D to full 3D, passing through a sequence of quasi-2D well configurations. The above results can be viewed as an extension of, or more appropriately, as an exact quantal correction to, the extremal free-electron cross-sections picture, usually employed in this problem [21-23].

We can also note that with the above analytical solution, one can extend calculations to the derivation of other (thermodynamic) properties of the interface, such as pressure or compressibility, by taking proper derivatives with respect to volume (for constant $N$ ); however, this is something that we will not pursue here.

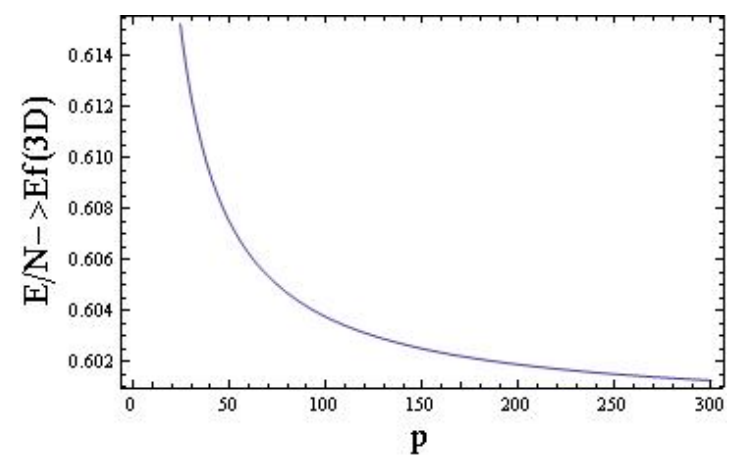

Fig. 3.3: Energy (in units of 3D Fermi energy) as a function of $z$-levels quantum number. Note that for approximately more than $300 z$-axis occupied levels, the total energy tends rapidly to $E / N=3 / 5 E f(3 D)$. 


\section{Finite-thickness interface in a perpendicular magnetic field}

In the previous example of the finite- $d$ interface, the single-particle energies were quantised only in the $z$ direction and they were quasicontinuous in the $x y$ plane. Later in Section 5, where the full 3D problem in a magnetic field is considered, we will find single-particle energies that will only be quantised in the $x y$ plane (Landau levels) and will be quasicontinuous in the $z$-direction. In all these cases, we have quasicontinuity in at least one direction, such that the above discussed "equilibrium conditions" can continuously be satisfied, giving at every point, i.e., for every density, the optimal partition or arrangement of our Fermionic particles in singleparticle states. One might wonder how the above method could be used if the single-particle energy is strongly quantised in all directions. This is actually the case of our main interest, namely when we consider a finitethickness interface inside a perpendicular magnetic field. In such a case, the previous equilibrium condition is not satisfied in a continuous way, as there are no quasicontinuous Fermi circles (of Section 3) or Fermi line segments (of Section 5). We now do not quite have equilibrium equalities at every density, as in the other two problems but we rather have inequalities that change directions in a discrete manner with variation of density, which actually determine the lowest-energy occupation scenario. However, we will still have distinct points of transition (into different occupation scenarios) whenever certain equalities are again satisfied, as we will see. Specifically, it will transpire that to determine these equalities, requires a close and careful study and that there is no simple analytical solution that can be written directly for a generic $B$ and $d$, even though we are dealing with noninteracting electrons at $\mathrm{T}=0$, i.e., the energy cannot be written directly in closed form for an arbitrary field and thickness - one has to actually run the occupation procedure carefully for all "previous" values of $B$ and $d$ starting from easy limiting cases, unlike the other two problems. The interplay between the strong quantisation in the $x y$ plane and the simultaneous strong quantisation in the $z$-axis leads to rather unpredictable patterns under combined variations of $B$ and $d$, when one simply occupies one-electron states in a manner that maintains the lowest possible total energy.

The single-electron spectrum is now given by

$$
\varepsilon\left(n, n_{z}\right)=\hbar \omega_{c}\left(n+\frac{1}{2}\right)+\varepsilon_{n z}
$$

where $n$ is again the Landau level index $(n=0,1,2 \ldots)$ and the QW-levels are again represented by

$$
\varepsilon_{n z}=\frac{\hbar^{2} k_{z}^{2}}{2 m}, \quad \text { where } \quad k_{z}=\frac{\pi n_{z}}{d}, \quad n_{z}=1,2,3, \ldots
$$

Let us first see a simple example of the above-mentioned competitions that are expressed with inequalities. If $d$ is extremely small (to be further quantified below), then for a given $B$ (not very strong - such that there are more than one LLs needed (see below)), it is energetically favourable for the electrons to be placed in several distinct LLs consecutively, starting from the lowest and moving upwards in energy until all the electrons of the system are accommodated and to keep the system "frozen" in the $n_{z}=1 \mathrm{QW}$-level. In such a case, the problem is essentially equivalent to the $2 \mathrm{D}$ problem of Section 2, apart from an extra constant term in the energy (i.e., common to all electrons) due to the QW confinement. However, if the thickness $d$ starts increasing, then there might come a point (in density) when an extra electron would energetically prefer to be placed in $n_{z}=2$ and start to occupy from the beginning a lower LL, which is already occupied by other electrons that correspond to $n_{z}=1$ without violating Pauli's principle (note that, apart from $n$ and $n_{z}$, the $3^{\text {rd }}$ integer $l$ is already implicitly used, counting the degenerate states for each combined pair $\left(n, n_{z}\right)$; therefore, it does not need to be mentioned in any special way). The simplest nontrivial case is when two lowest LLs, i.e., $n=0$ and $n=1$, are originally involved (for $n_{z}=1$ ) and then, upon an increase of $d$, the above transition to $n_{z}=2$ and back to $n=0$ only takes place; this transition will happen when

$$
\frac{\hbar \omega_{c}}{2}+\boldsymbol{\varepsilon}_{n z}=2=\frac{3 \hbar \omega_{c}}{2}+\boldsymbol{\varepsilon}_{n z}=1
$$

This is in the spirit of "equilibrium" that was used earlier in (3.5), although here, it occurs in steps for discrete values of parameters. Once again, the extra particle that is about to be accommodated according to various possible occupation scenarios, has a single-particle energy that must be the same in all of them; otherwise, the process would not be fair and it would lead to higher total energy [20]. (4.3) leads to a critical value of thickness $d$ where the transition occurs for a given $B$, namely 


$$
d_{c r i t}=\sqrt{\frac{3 \pi \Phi_{o}}{4 B}} .
$$

The above was only the simplest example in order to stress the essential point and to motivate what follows, the general case involving an arbitrary number of LLs and QWs still needs to be worked out. One then wonders what one can say in full generality for the correct partition (in combined $n$ and $n_{z}$ states) for arbitrary values of $B$ and $d$ for this problem. In the general case, there is "asymmetry" in the manner with which we need to treat $d-B$ variations, in order to have good control on all possible cases and better understanding of the patterns that occur. If, for example, one follows the route of having fixed $d$ and varying $B$, analogous to what is done in the 2D case (Section 2) but for a finite $d$ (the experimentally relevant route, of a given interface), then the problem is rather difficult to analyse systematically, producing results that sometimes look "surprising", i.e., new transitions appear in the interior of certain windows of $B$-values (windows with ends that are consistent with dHvA variations), the origin and location of these "internal transitions" not being easily identifiable. The point is that variation of $B$ for fixed $d$ changes not only the energetic distance between LLs but also their degeneracies and this interplay, together with the competition with the energetic distance between QW-levels, leads to a multitude of cases to be investigated that do not appear easily subdued to a systematic control. However, it transpires that the opposite route of temporarily keeping $B$ fixed and varying $d$ and then changing $B$ in a particular way and repeating the procedure of the variation of $d$, offers a much better control in the theoretical treatment; this is basically because degeneracies of each LL are fixed and we need only to focus on competitions between LL-QW energetic distances. Although the results are of course equivalent with both methods, what we called "surprising results" of the $1^{\text {st }}$ route will find a better understanding through the $2^{\text {nd }}$ route, both in terms of origin and location. In the following, we will pursue the $2^{\text {nd }}$ route for theoretical convenience but in the final figures, we will also show results as would appear from the $1^{\text {st }}$ method and later, we will also provide $2 \mathrm{D}$ figures that show the full results under combined variations of $B$ and $d$; the ordering then of what is kept fixed not being important.

Let us start being more quantitative and in accordance with the mathematically $2 \mathrm{D}$ problem of Section 2 , let us first assume that the number of electrons lies in the following window:

$$
N \leq 2 \frac{\Phi}{\Phi_{o}},
$$

such that only a single LL is involved, although now combined with a QW-level (see below). Always treating $N$ as fixed (so that $n_{A}=N / S$ is fixed as well), (4.5) is equivalent to

$$
B \geq \frac{1}{2} n_{A} \Phi_{o},
$$

where it should again be noted that the effective areal density $n_{A}=N / S$ is related to the volume density $n_{V}$ through $n_{A}=n_{V} d$ and $\Phi_{o}=h c / e$ is the flux quantum. Now, each quantum state is again characterised by three quantum numbers, namely $\left\{n, l, n_{z}\right\}$ with the positive integer $l$ counting the degenerate states inside an LL (or more appropriately, inside a combined $\left(n, n_{z}\right)$-pair) and taking $2 \Phi / \Phi o$ values, such that each combined pair $(n$, $n_{z}$ ) can contain up to $2 \Phi / \Phi$ o electrons (according to Pauli's Exclusion Principle). Then it is easy to see that, when (4.5) is satisfied, the electron system will occupy only one combined-pair, $\left\{n=0, n_{z}=1\right\}$, while $l$ runs from 1 up to $N$, which here, is less than the LL degeneracy $2 \Phi / \Phi o$ and this will give a total energy

$$
E=N \varepsilon\left\{n=0, n_{z}=1\right\}
$$

with $\varepsilon\left\{n=0, n_{z}=1\right\}$ the single-particle energy (4.1) with $n=0$ and $n_{z}=1$. We can write this energy in terms of 2D Fermi energy (corresponding to the absence of magnetic field), as

$$
\frac{E}{N}=E_{f}\left[\frac{B}{n_{A} \Phi_{o}}+\left(\frac{\pi}{2 n_{A} d^{2}}\right)\right]
$$

where $E_{f}=\frac{\hbar^{2} k_{f}^{2}}{2 m}=\frac{\hbar^{2} 2 \pi n_{A}}{2 m}$.

That is, if $B$ satisfies (4.6) then, for every value of thickness $d$, electrons occupy only the states with the lowest possible quantum numbers $n$ and $n_{z}$ (see Fig. 4.1 - note that in this and all following figures we simply compare 
single-particle energy differences, by always placing at the same level the beginning of each energy difference that needs to be compared. In such a way, the comparison is visually obvious; therefore, the placement of the levels does not have an absolute meaning in energy and only the differences matter).

Fig. 4.1: Occupied states for every $d$

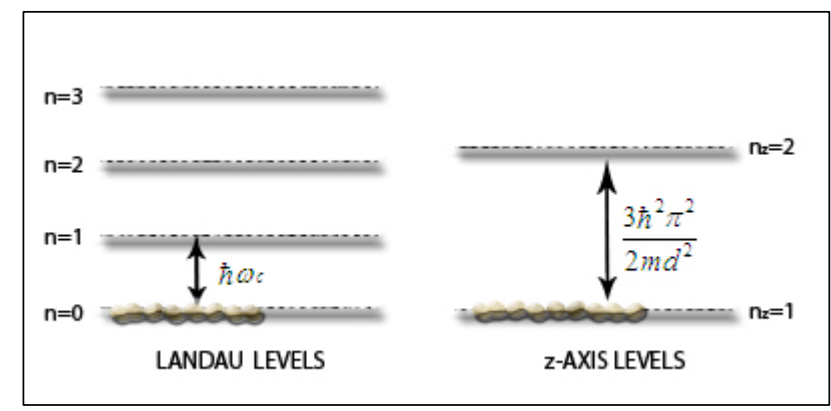

Let us now start lowering $B$. The next window of $B$-values, a natural choice if we follow the 2D paradigm of Section 2, is

$$
4 \frac{\Phi}{\Phi_{o}} \geq N \geq 2 \frac{\Phi}{\Phi_{o}} \quad \text { or } \quad \frac{1}{4} n_{A} \Phi_{o} \leq B \leq \frac{1}{2} n_{A} \Phi_{o}
$$

Now the usual occupation scenario would normally be the one in which the extra $N-2 \Phi / \Phi_{0}$ electrons will need to be placed in the next LL (the one with $n=1$ ) as in Section 2 but this is not necessarily true. It might be energetically favourable for some electrons to occupy another QW-level with respect to Pauli's principle, because of the extra degree of freedom provided by $n_{z}$. We can see immediately the possible options; the two appropriate possibilities are $\left\{n=1, n_{z}=1\right\}$ (increase $n$ by 1 ) or $\left\{n=0, n_{z}=2\right\}$ (increase $n_{z}$ by 1 and go back to the lowest LL). However, which one is the correct one and under what conditions? The answer is that this will be determined by the thickness of the sample. Let us try to find the critical thickness at which the two possibilities lead to the same single-particle energy:

$$
\varepsilon\left\{n=1, n_{z}=1\right\}=\varepsilon\left\{n=0, n_{z}=2\right\},
$$

which is (4.3) that we saw earlier as a motivating example, or equivalently

$$
\hbar \omega_{c}=\frac{3 \hbar^{2} \pi^{2}}{2 m d^{2}}
$$

which in turn leads to

$$
\left.\Rightarrow d_{c r i t}=\sqrt{\frac{3 \pi \Phi_{o}}{4 B}} \quad \text { (the same as }(4.4)\right) \text {. }
$$

Note that the critical thickness depends on the value of the magnetic field, as long as this field lies inside the window of (4.9). It is easy to see that for values of thickness lower than (4.12), it is the states $\left\{n=1, n_{z}=1\right\}$ (always meaning for all $0<l<N-2 \Phi / \Phi_{0}$ ) that are occupied by the excess electrons (see Fig. 4.2), i.e., the system behaving like a $2 \mathrm{D}$ system and for values of $d$ greater than (4.12), it is the states $\left\{n=0, n_{z}=2\right\}$ that are occupied by the excess electrons, because the energy difference $\varepsilon_{n z}=2-\varepsilon_{n z=1}$ is smaller that $\hbar \omega_{c}$ (see Fig. 4.3). In addition, it is interesting to note that for exactly $B=1 / 2 n_{A} \Phi_{o}$, then $d_{c r i t}=\sqrt{3 \pi / 2 n_{A}}$, which is exactly the critical thickness (3.6) of Section 3 that gives the criterion for the 2-dimensionality of the system. 
In the figures below, where the relevant information is visually presented, the arrows denote the LLs that are combined with QW levels and their common filling is represented by the filling of corresponding boxes. [Note that the number of arrows that combine states is the same for every window of $B$-values that we study (as fixed) in what follows and is not necessarily equal to the number of LLs and/or the number of QWs involved, as will be seen by later examples.]

Table 1: Occupied states

$$
\frac{1}{4} n_{A} \Phi_{o} \leq B \leq \frac{1}{2} n_{A} \Phi_{o}
$$

\section{Thickness values}

$d \leq \sqrt{\frac{3 \pi \Phi_{o}}{4 B}}$
$d \geq \sqrt{\frac{3 \pi \Phi_{o}}{4 B}}$
Occupied states

$\left\{n=0, n_{z}=1\right\},\left\{n=1, n_{z}=1\right\}$

$\left\{n=0, n_{z}=1\right\},\left\{n=0, n_{z}=2\right\}$

\section{Schematic representation:}

Fig. 4.2: $d \leq \sqrt{\frac{3 \pi \Phi_{o}}{4 B}}$

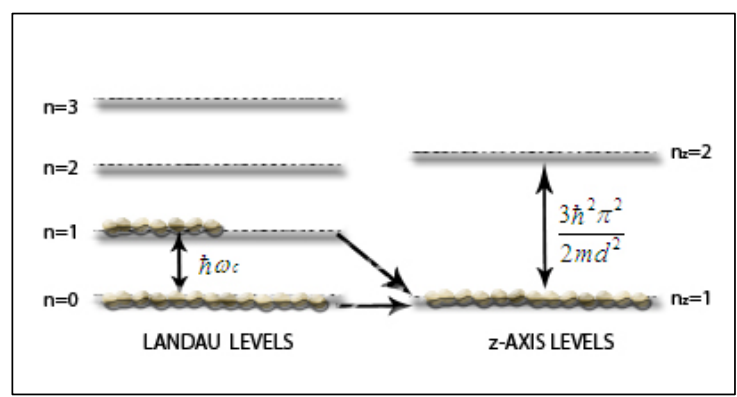

Fig. 4.3: $d \geq \sqrt{\frac{3 \pi \Phi_{o}}{4 B}}$

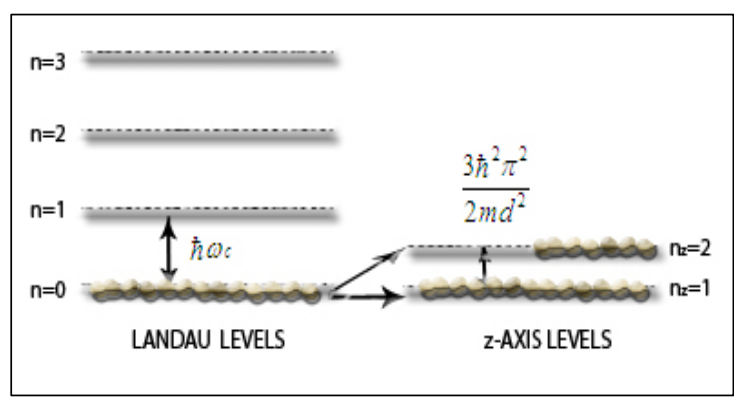

Following the above, it is now straightforward to write the total energy of the system for $\frac{1}{4} n_{A} \Phi_{o} \leq B \leq \frac{1}{2} n_{A} \Phi_{o}$. For every value of $B$ in this window, the total energy depends on $d$, according to:

$$
\begin{gathered}
d \leq \sqrt{\frac{3 \pi \Phi_{o}}{4 B}} \quad E=\frac{2 \Phi}{\Phi_{o}} \varepsilon\left\{n=0, n_{z}=1\right\}+\left(N-\frac{2 \Phi}{\Phi_{o}}\right) \varepsilon\left\{n=1, n_{z}=1\right\} \\
d \geq \sqrt{\frac{3 \pi \Phi_{o}}{4 B}} \quad E=\frac{2 \Phi}{\Phi_{o}} \varepsilon\left\{n=0, n_{z}=1\right\}+\left(N-\frac{2 \Phi}{\Phi_{o}}\right) \varepsilon\left\{n=0, n_{z}=2\right\},
\end{gathered}
$$

or in units of 2D Fermi energy

$$
\begin{aligned}
& d \leq \sqrt{\frac{3 \pi \Phi_{o}}{4 B}}: \quad \frac{E}{N}=E_{f}\left[-4\left(\frac{B}{n_{A} \Phi_{o}}\right)^{2}+3\left(\frac{B}{n_{A} \Phi_{o}}\right)+\left(\frac{\pi}{2 n_{A} d^{2}}\right)\right] \\
& d \geq \sqrt{\frac{3 \pi \Phi_{o}}{4 B}}: \quad \frac{E}{N}=E_{f}\left[\left(\frac{B}{n_{A} \Phi_{o}}\right)-6\left(\frac{B}{n_{A} \Phi_{o}}\right)\left(\frac{\pi}{2 n_{A} d^{2}}\right)+\left(\frac{4 \pi}{2 n_{A} d^{2}}\right)\right] .
\end{aligned}
$$


Let us now proceed further and present the third window of $B$-values, namely

$$
\frac{1}{6} n_{A} \Phi_{o} \leq B \leq \frac{1}{4} n_{A} \Phi_{o}
$$

A similar line of reasoning must then be followed. Starting with small values of thickness $d$, the system occupies only distinct LLs and is restricted to the lowest state in the $z$-axis. This prompts the question; how small must the thickness be for this to be the case? The answer is when the energy gap of the first two QW-levels is larger than $2 \hbar \omega_{c}$, because then the system is energetically favoured to occupy only distinct LLs. [Note, the difference from the previous cases where the QW difference should be compared with $\hbar \omega_{c}$ rather than $2 \hbar \omega_{c}$, e.g., see (4.11).] The first critical value of thickness where this is violated is determined by

and is equal to

$$
2 \hbar \omega_{c}=\frac{3 \hbar^{2} \pi^{2}}{2 m d^{2}}
$$

$$
d_{c r i t 1}=\sqrt{\frac{3 \pi \Phi_{o}}{4.2 . \mathrm{B}}}
$$

Note, that it again depends on the value of the magnetic field and it is also interesting to note that, if the field is exactly $B=1 / 4 n_{A} \Phi_{o}$ then we find again

$$
d_{c r i t}(2 D)=\sqrt{\frac{3 \pi}{2 n_{A}}}
$$

which is nothing but the $2 \mathrm{D}$ criterion (3.6) that we found earlier.

Fig. 4.4: $d<d_{c r i t 1}$

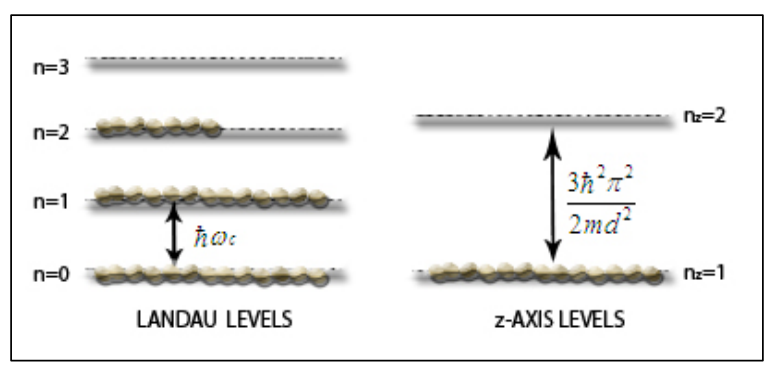

Fig. 4.5: $d=d_{c r i t 1}$

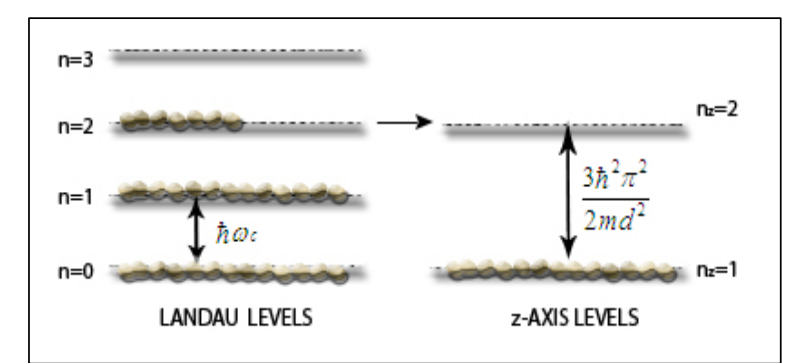

If we continue increasing the thickness beyond this first critical value, the electrons start the occupation of the second QW-level and this happens when

$$
2 \hbar \omega_{c}>\frac{3 \hbar^{2} \pi^{2}}{2 m d^{2}}
$$

In this case, the electrons in the incompletely filled LL state $n=2$ are falling in the state $n=0$, losing energy $2 \hbar \omega_{c}$. Simultaneously, the same electrons are excited from QW-level $n_{z}=1$ to $n_{z}=2$, gaining energy $3 \hbar^{2} \pi^{2} / 2 m d^{2}$. The energy gained by this procedure is of course lower than that lost, owing to (4.21), thus, making this transition energetically favoured (see Fig. 4.6).

The next transition (upon further increase of $d$ ) occurs when the gap between the two first QW levels drops to the value $\hbar \omega_{c}$ of the Landau gap, namely:

$$
\hbar \omega_{c}=\frac{3 \hbar^{2} \pi^{2}}{2 m d^{2}} \Rightarrow d_{c r i t 2}=\sqrt{\frac{3 \pi \Phi_{o}}{4 \mathrm{~B}}} .
$$


Fig. 4.6: $d>d_{c r i t 1}$

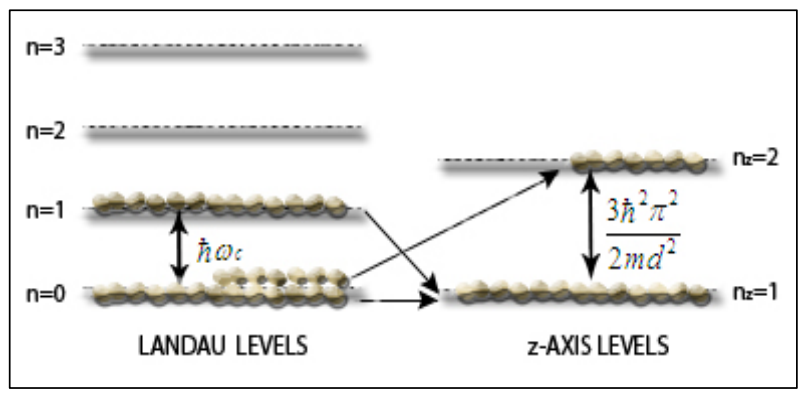

Fig. 4.7: $\quad d=d_{\text {crit } 2}$

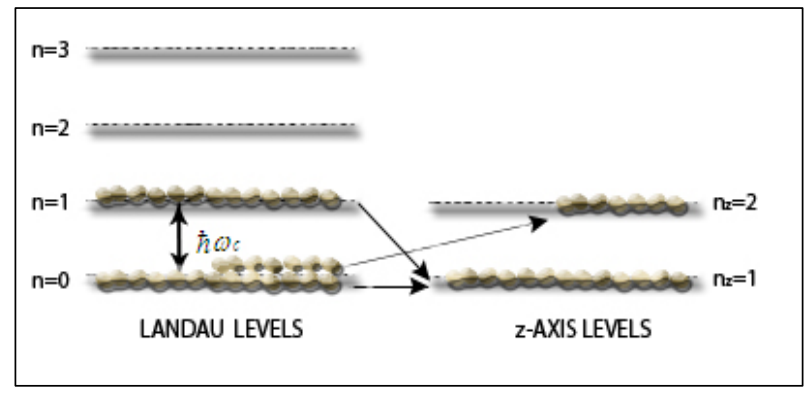

If the thickness is increased further, then the following relation holds:

$$
\hbar \omega_{c}>\frac{3 \hbar^{2} \pi^{2}}{2 m d^{2}}
$$

Some of the electrons of the $n=1 \mathrm{LL}$ will then fall on the $n=0$ LL (by now fully occupying it) and at the same time they are excited in the $n_{z}=2$ QW-level, leaving the $n=1$ LL partially occupied (see Fig. 4.8).

However, there is still one more qualitatively distinct scenario before we get to the end of this procedure. With a further increase of thickness, we find the following relation

$$
\hbar \omega_{c}=\frac{8 \hbar^{2} \pi^{2}}{2 m d^{2}} \Rightarrow d_{c r i t 3}=\sqrt{\frac{8 \pi \Phi_{o}}{4 \mathrm{~B}}}
$$

which happens when the $n_{z}=3$ level has fallen so low that the difference $\varepsilon_{n z}=3-\boldsymbol{\varepsilon}_{n z}=1$ is equal to $\hbar \omega_{c}$. Then, above this value of thickness, we only have the lowest LL combined with the three lowest QW-levels (see Fig. 4.10).

Fig. 4.8: $d>d_{\text {crit } 2}$

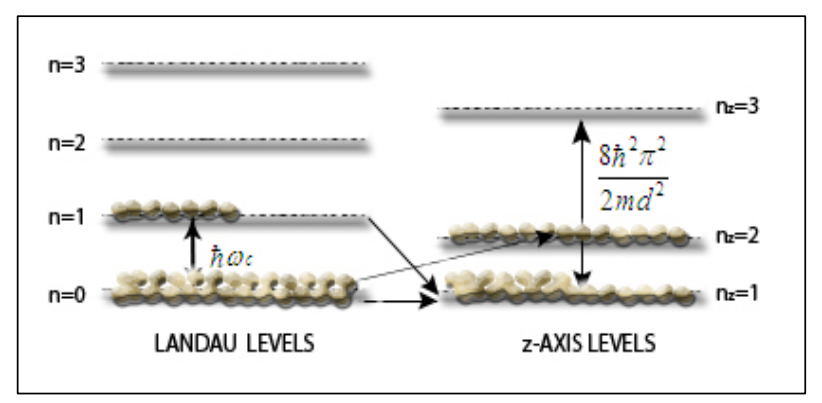

Fig. 4.9: $d=d_{c r i t 3}$




Fig. 4.10: $d>d_{c r i t 3}$

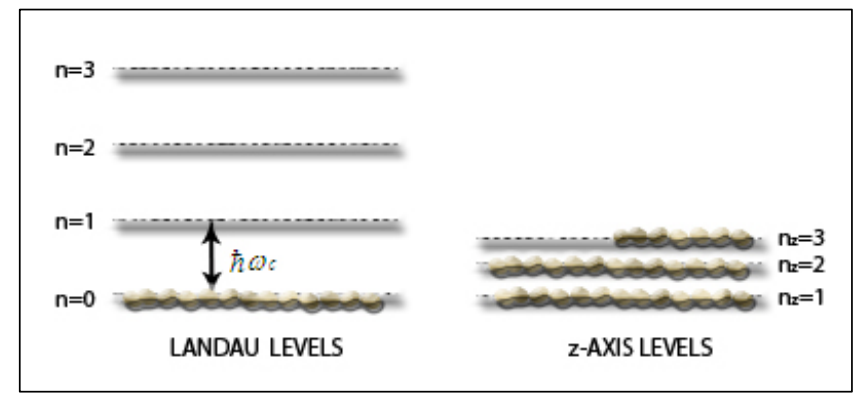

A summary of all the occupation scenarios for $1 / 6 n_{A} \Phi_{o} \leq B \leq 1 / 4 n_{A} \Phi_{o}$ is shown in Table 2 and the corresponding total energies for all the various windows of $d$-values are given in Table 3.

Table 2: Occupied states

$$
\frac{1}{6} n_{A} \Phi_{o} \leq B \leq \frac{1}{4} n_{A} \Phi_{o}
$$

Window of $d$-values

$$
\text { Occupied states }
$$

$$
\begin{array}{rlrl}
d & \leq \sqrt{\frac{3 \pi \Phi_{o}}{4 \cdot 2 \cdot B}} & \left\{n=0, n_{z}=1\right\},\left\{n=1, n_{z}=1\right\},\left\{n=2, n_{z}=1\right\} \\
\sqrt{\frac{3 \pi \Phi_{o}}{4.1 . B}} \geq d \geq \sqrt{\frac{3 \pi \Phi_{o}}{4 \cdot 2 \cdot B}} & \left\{n=0, n_{z}=1\right\},\left\{n=1, n_{z}=1\right\},\left\{n=0, n_{z}=2\right\} \\
\sqrt{\frac{8 \pi \Phi_{o}}{4 B}} \geq d \geq \sqrt{\frac{3 \pi \Phi_{o}}{4.1 . B}} & \left\{n=0, n_{z}=1\right\},\left\{n=0, n_{z}=2\right\},\left\{n=1, n_{z}=1\right\} \\
d & \geq \sqrt{\frac{8 \pi \Phi_{o}}{4 B}} & \left\{n=0, n_{z}=1\right\},\left\{n=0, n_{z}=2\right\},\left\{n=0, n_{z}=3\right\}
\end{array}
$$

Table 3: Total energies

$$
\frac{1}{6} n_{A} \Phi_{o} \leq B \leq \frac{1}{4} n_{A} \Phi_{o}
$$

Window of $d$-values

$$
\begin{gathered}
d \leq \sqrt{\frac{3 \pi \Phi_{o}}{4.2 . B}} \\
\sqrt{\frac{3 \pi \Phi_{o}}{4.1 . B}} \geq d \geq \sqrt{\frac{3 \pi \Phi_{o}}{4.2 . B}} \\
\sqrt{\frac{8 \pi \Phi_{o}}{4 B}} \geq d \geq \sqrt{\frac{3 \pi \Phi_{o}}{4.1 . B}} \\
d \geq \sqrt{\frac{8 \pi \Phi_{o}}{4 B}}
\end{gathered}
$$$$
\frac{E}{N}=E_{f}\left[-12\left(\frac{B}{n_{A} \Phi_{o}}\right)^{2}+5\left(\frac{B}{n_{A} \Phi_{o}}\right)+\left(\frac{\pi}{2 n_{A} d^{2}}\right)\right]
$$$$
\frac{E}{N}=E_{f}\left[4\left(\frac{B}{n_{A} \Phi_{o}}\right)^{2}+\left(\frac{B}{n_{A} \Phi_{o}}\right)-12\left(\frac{B}{n_{A} \Phi_{o}}\right)\left(\frac{\pi}{2 n_{A} d^{2}}\right)+\left(\frac{4 \pi}{2 n_{A} d^{2}}\right)\right]
$$$$
\frac{E}{N}=E_{f}\left[-8\left(\frac{B}{n_{A} \Phi_{o}}\right)^{2}+3\left(\frac{B}{n_{A} \Phi_{o}}\right)+6\left(\frac{B}{n_{A} \Phi_{o}}\right)\left(\frac{\pi}{2 n_{A} d^{2}}\right)+\left(\frac{\pi}{2 n_{A} d^{2}}\right)\right]
$$$$
\frac{E}{N}=E_{f}\left[\left(\frac{B}{n_{A} \Phi_{o}}\right)-26\left(\frac{B}{n_{A} \Phi_{o}}\right)\left(\frac{\pi}{2 n_{A} d^{2}}\right)+\left(\frac{9 \pi}{2 n_{A} d^{2}}\right)\right]
$$ 
Let us also study the next window of $B$-values, namely:

$$
\frac{1}{8} n_{A} \Phi_{o} \leq B \leq \frac{1}{6} n_{A} \Phi_{o}
$$

because there are some special elements showing up, signifying the non-integrability of this problem. We will now be more compact and will present in figures essentially an animation of what happens as the thickness $d$ is continuously increased, always for a fixed value of $B$ that lies inside the window (4.25).

Fig. 4.11: $d \leq \sqrt{\frac{3 \pi \Phi_{o}}{4.3 \cdot B}}$

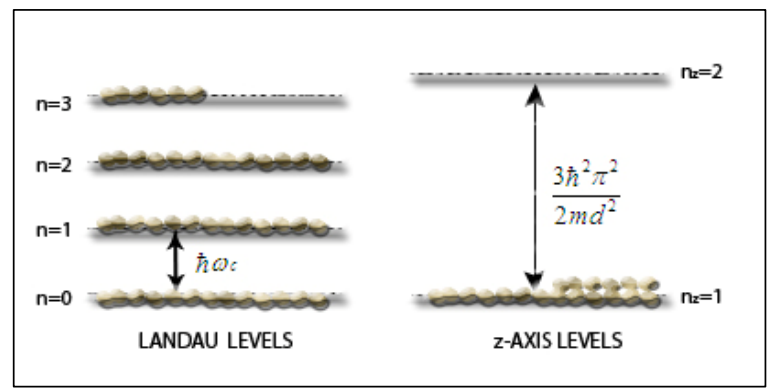

Only the lowest QW is occupied because $\Delta \mathrm{E}_{z}\{1,2\}>3 \hbar \omega_{c}$

Fig. 4.13: $d>\sqrt{\frac{3 \pi \Phi_{o}}{4.3 \cdot B}}$

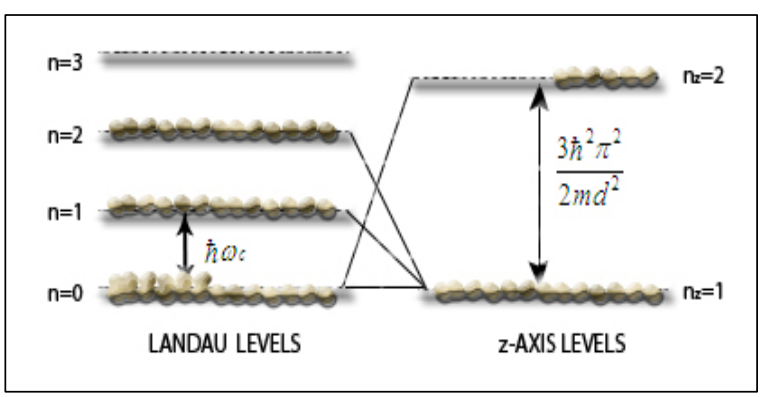

States $\{n=3, n z=1\}$ are abandoned and $\left\{\mathrm{n}=0, \mathrm{nz}_{\mathrm{z}}=2\right\}$ are partially occupied because $\Delta \mathrm{E}_{z}\{1,2\}<3 \hbar \omega_{c}$
Fig. 4.12: $d=\sqrt{\frac{3 \pi \Phi_{o}}{4.3 \cdot B}}$

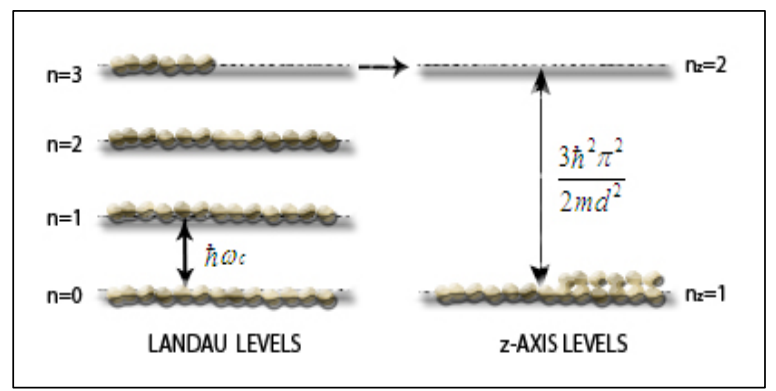

The equality $\Delta \mathrm{E}_{z}\{1,2\}=3 \hbar \omega_{c}$ is satisfied

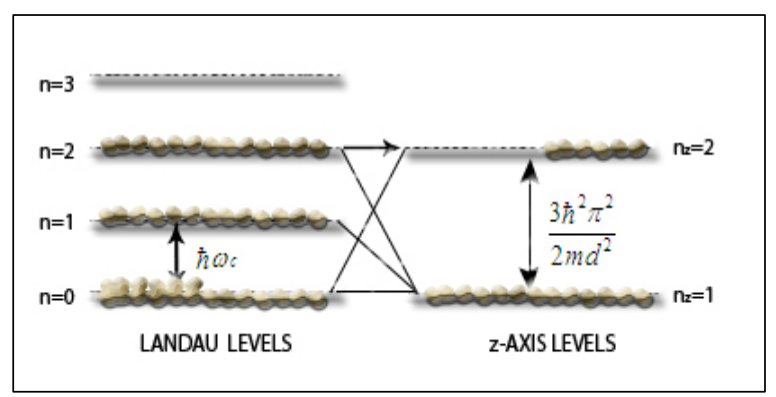

The equality $\Delta \mathrm{E}_{z}\{1,2\}=2 \hbar \omega_{c}$ is satisfied 
Fig. 4.15: $d>\sqrt{\frac{3 \pi \Phi_{o}}{4.2 . B}}$

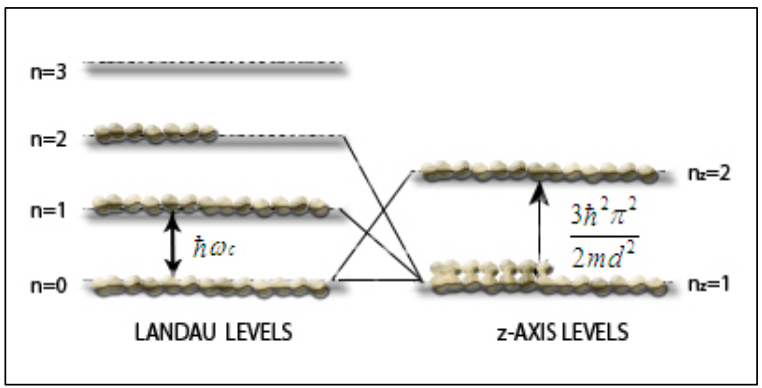

States $\{n=0, n z=2\}$ are fully occupied and $\{n=2, n z=1\}$ are only partially filled because $\Delta \mathrm{E}_{z}\{1,2\}<2 \hbar \omega_{c}$

Fig. 4.17: $d>\sqrt{\frac{3 \pi \Phi_{o}}{4.1 . B}}$

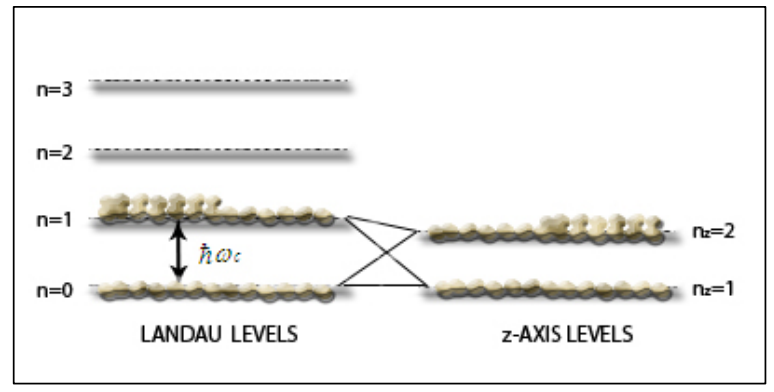

States $\left\{n=2, n_{z}=1\right\}$ are abandoned and $\left\{n=1, n_{z}=2\right\}$ are partially occupied because $\Delta \mathrm{E}_{z}\{1,2\}<\hbar \omega_{c}$

Fig. 4.19: $d>\sqrt{\frac{5 \pi \Phi_{o}}{4 B}}$

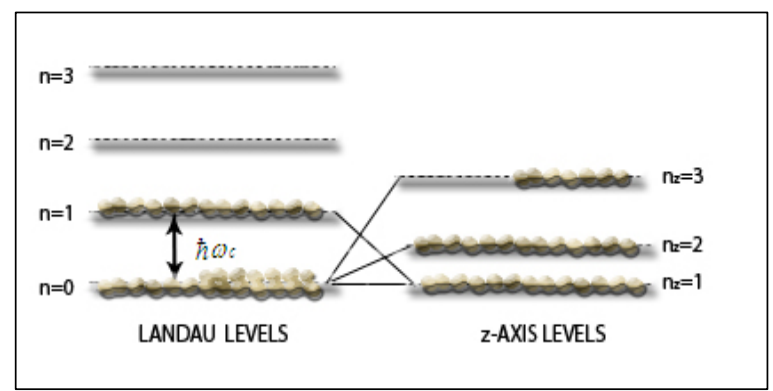

States $\left\{n=1, n_{z}=2\right\}$ are abandoned and $\left\{n=0, n_{z}=3\right\}$ are partially occupied because $\Delta \mathrm{E}_{z}\{3,2\}<\hbar \omega_{c}$
Fig. 4.16: $d=\sqrt{\frac{3 \pi \Phi_{o}}{4.1 . B}}$

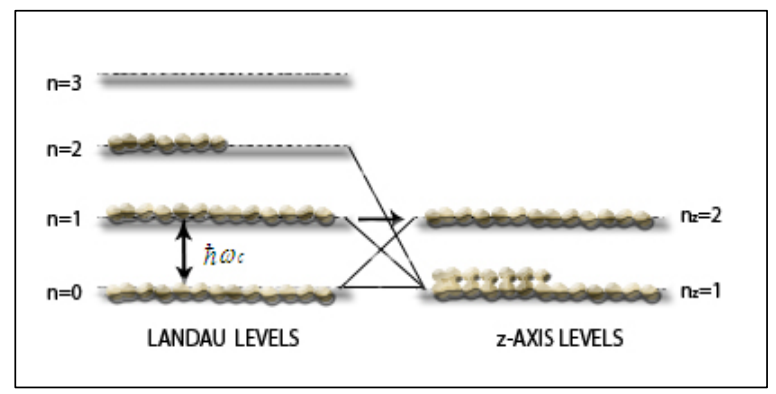

The equality $\Delta \mathrm{E}_{z}\{1,2\}=\hbar \omega_{c}$ is satisfied

Fig. 4.18: $d=\sqrt{\frac{5 \pi \Phi_{o}}{4 B}}$

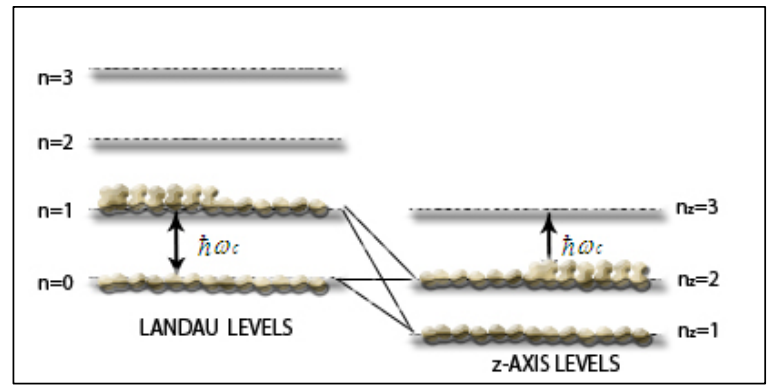

The equality $\Delta \mathrm{E}_{z}\{3,2\}=\hbar \omega_{c}$ is satisfied. (Note that we now have to compare between states that have $n_{z}$ greater than 1)

Fig. 4.20: $d=\sqrt{\frac{8 \pi \Phi_{o}}{4 B}}$

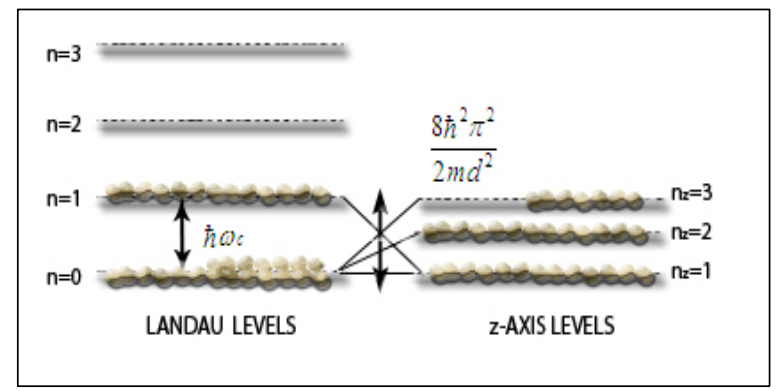

The equality $\Delta \mathrm{E}_{z}\{3,1\}=\hbar \omega_{c}$ is satisfied 
Fig. 4.21: $d>\sqrt{\frac{8 \pi \Phi_{o}}{4 B}}$

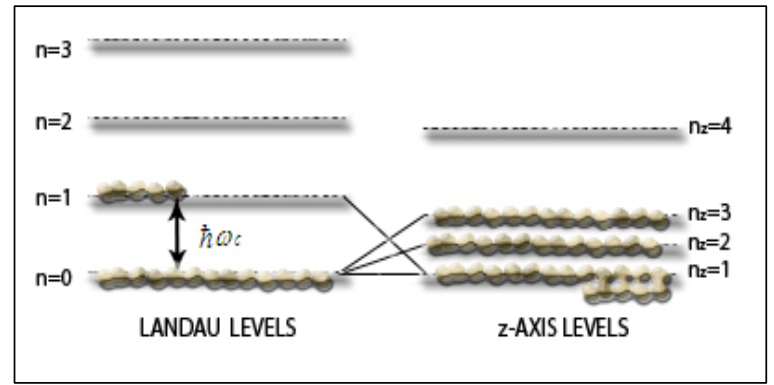

States $\{n=0, n z=3\}$ are fully occupied and $\{n=1, n z=1\}$ are partially filled because $\Delta \mathrm{E}_{z}\{3,1\}<\hbar \omega_{c}$
Fig. 4.22: $d=\sqrt{\frac{15 \pi \Phi_{o}}{4 B}}$

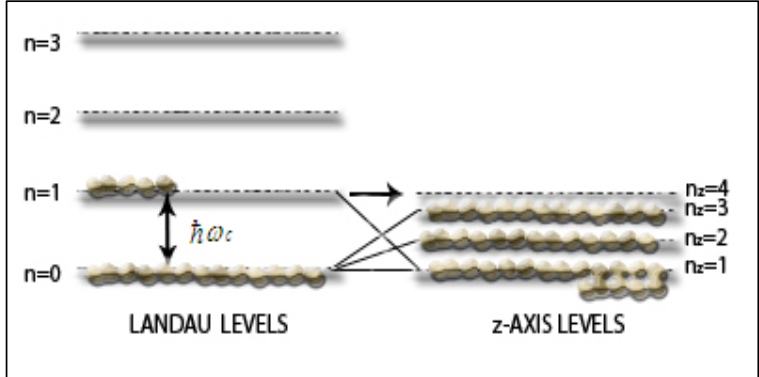

The equality $\Delta \mathrm{E}_{z}\{4,1\}=\hbar \omega_{c}$ is satisfied

Fig. 4.23: $d>\sqrt{\frac{15 \pi \Phi_{o}}{4 B}}$

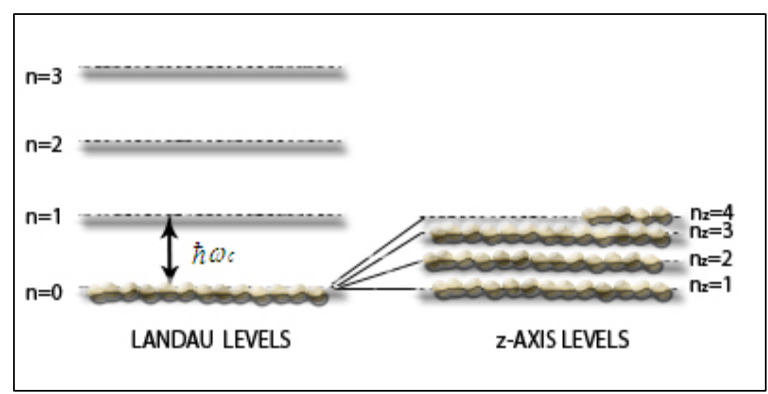

Only the lowest LL states are occupied, combined with all $4 z$-axis levels, because $\Delta \mathrm{E}_{z}\{4,1\}<\hbar \omega_{c}$

From the above examples, one should note that sometimes, in intermediate steps, the energetics involve competition between the LLs and QW levels that are not necessarily the lowest possible, e.g., in Fig. 4.18, where in the competition of energy differences it was the levels $n z=2$ and $n z=3$ that were involved and not the lowest $n z=1$ level. This is because the $n z=1$ level has already been combined with both available LLs and there is no extra freedom for this level to be involved any more. Note again that the energy comparisons in all the above figures are made only for energy differences that stand side to side and not for the absolute energy values. If we wanted the absolute spectrum, we would have to add the two contributions and then we would have crossovers at the points of transitions; it is actually in this form of crossover that we will detect possible effects of the above type later in Section 6 on topological insulators. More subtle behaviours in the energetic comparisons such as this one, will be seen in the examples that follow and these give to the results a certain form of unpredictability, because they are only determined by the system itself when the occupational procedure is run, under the energy criteria set up earlier and the Pauli Exclusion Principle. This leads to an interesting pattern of possible occupation scenarios, which have corresponding consequences on measurable quantities that will be shown later.

Let us finally present the results for the fifth window of $B$-values that involve comparisons between the LLs and QW levels that are slightly more complex, which is:

$$
\frac{1}{10} n_{A} \Phi_{o} \leq B \leq \frac{1}{8} n_{A} \Phi_{o} .
$$

As the number of windows of $d$-values turns out to be rather large (21), we will present this case only with figures, as we did before but with no commentary. One should again observe that not all cases refer to comparisons between the lowest LL and QW levels. 
Fig. 4.24: $d \leq \sqrt{\frac{3 \pi \Phi_{o}}{4.4 . B}}$

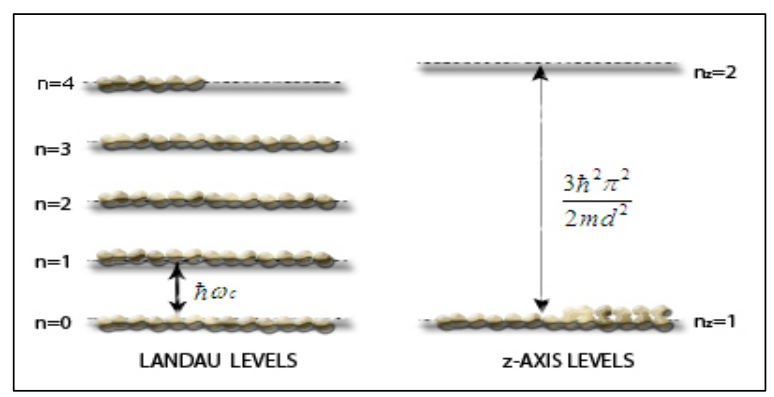

Only lowest QW is occupied because $\Delta \mathrm{E}_{z}\{1,2\}>4 \hbar \omega_{c}$

Fig. 4.26: $d \geq \sqrt{\frac{3 \pi \Phi_{o}}{4.4 . B}}$

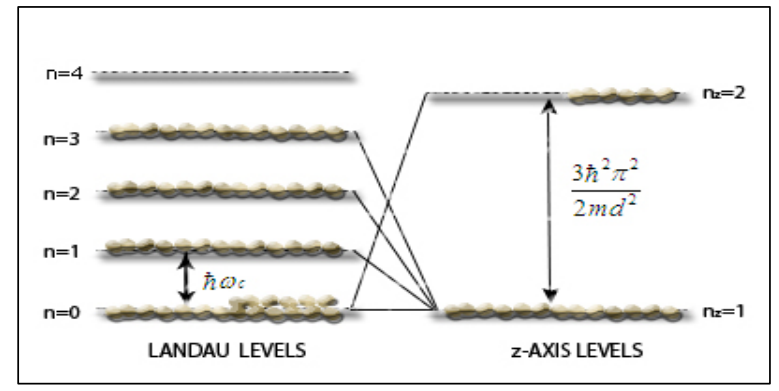

States $\{n=4, n z=1\}$ are abandoned and states $\left\{n=0, n_{z}=2\right\}$ are occupied because $\Delta \mathrm{E}_{z}\{1,2\}<4 \hbar \omega c$

Fig. 4.28: $d \geq \sqrt{\frac{3 \pi \Phi_{o}}{4.3 \cdot B}}$

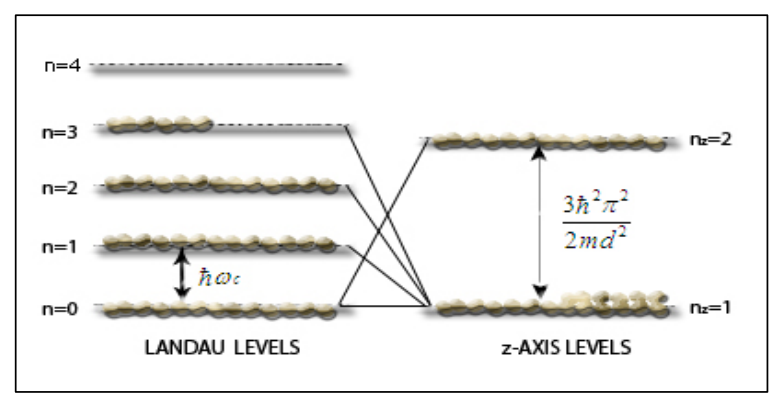

States $\{n=0, n z=2\}$ are fully occupied, while $\{n=3, n z=1\}$ are partially filled because $\Delta \mathrm{E}_{z}\{1,2\}<3 \hbar \omega_{c}$
Fig. 4.25: $d=\sqrt{\frac{3 \pi \Phi_{o}}{4.4 . B}}$

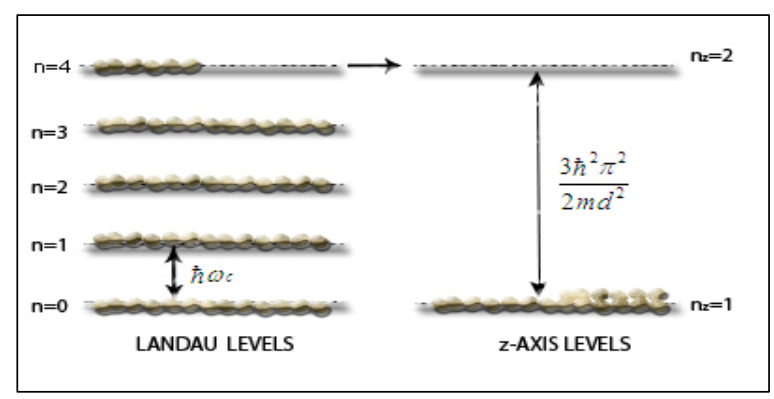

The equality $\Delta \mathrm{E}_{z}\{1,2\}=4 \hbar \omega_{c}$ is satisfied

Fig. 4.27: $d=\sqrt{\frac{3 \pi \Phi_{o}}{4.3 \cdot B}}$

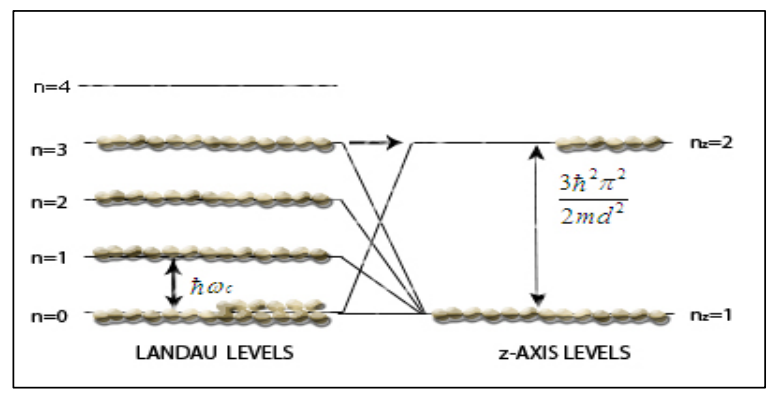

The equality $\Delta \mathrm{E}_{z}\{1,2\}=3 \hbar \omega_{c}$ is satisfied

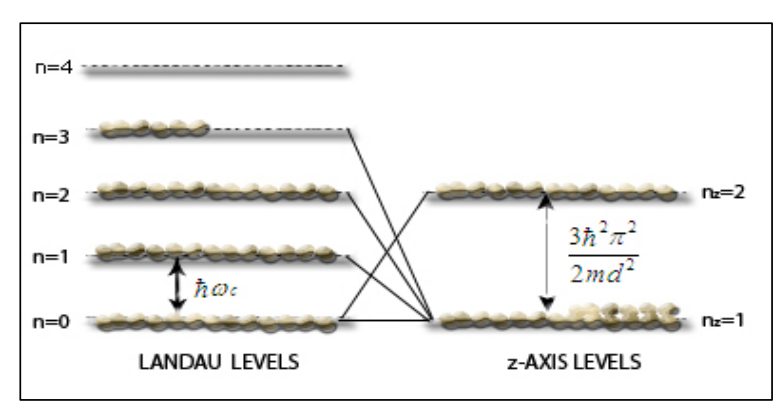

The equality $\Delta \mathrm{E}_{z}\{1,2\}=2 \hbar \omega_{c}$ is satisfied 
Fig. 4.30: $d \geq \sqrt{\frac{3 \pi \Phi_{o}}{4.2 . B}}$

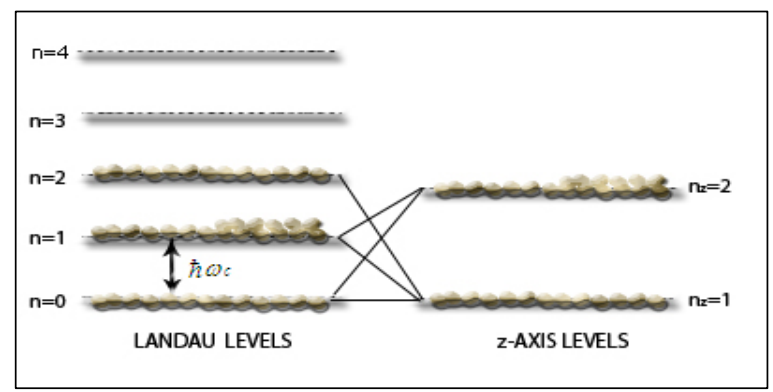

States $\left\{n=3, n_{z}=1\right\}$ are abandoned and $\left\{n=1, n_{z}=2\right\}$ are partially occupied because $\Delta \mathrm{E}_{z}\{1,2\}<2 \hbar \omega_{c}$

Fig. 4.32: $d \geq \sqrt{\frac{3 \pi \Phi_{o}}{4 B}}$

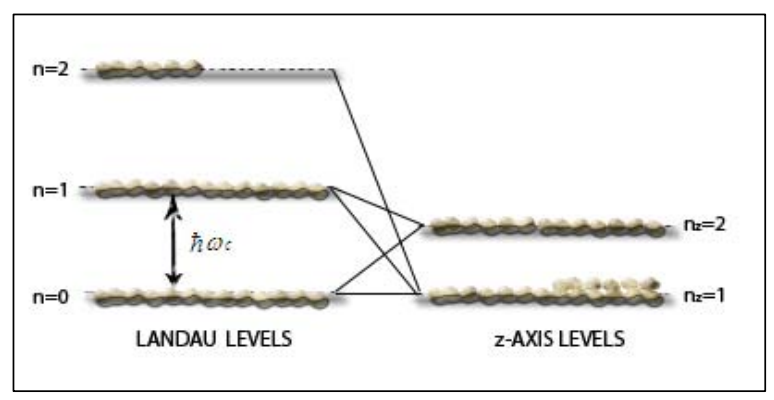

States $\left\{n=1, n_{z}=2\right\}$ are fully occupied, while $\left\{n=2, n_{z}=1\right\}$ are now partially filled because $\Delta \mathrm{E}_{z}\{1,2\}<\hbar \omega_{c}$

Fig. 4.34: $d \geq \sqrt{\frac{4 \pi \Phi_{o}}{4 B}}$

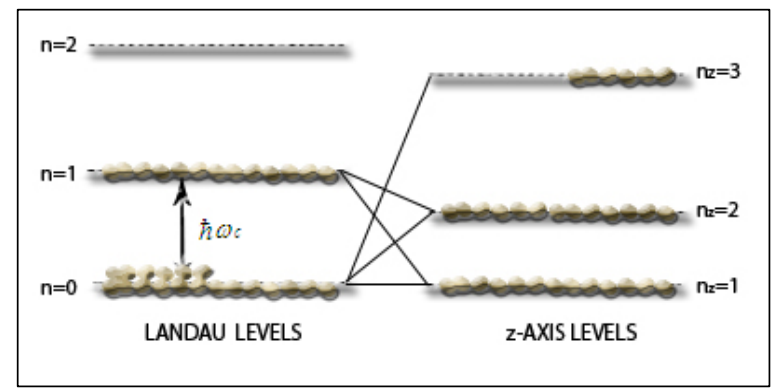

States $\left\{n=2, n_{z}=1\right\}$ are abandoned and $\left\{n=0, n_{z}=3\right\}$ are now occupied because $\Delta \mathrm{E}_{z}\{3,1\}<2 \hbar \omega_{c}$
Fig. 4.31: $d=\sqrt{\frac{3 \pi \Phi_{o}}{4 B}}$

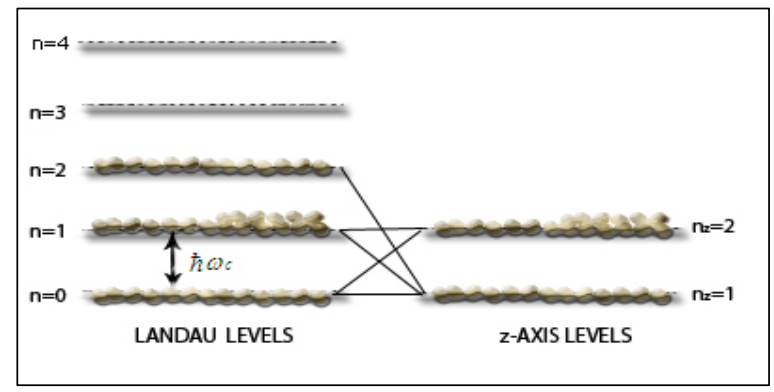

The equality $\Delta \mathrm{E}_{z}\{1,2\}=\hbar \omega_{c}$ is satisfied

Fig. 4.33: $d=\sqrt{\frac{4 \pi \Phi_{o}}{4 B}}$

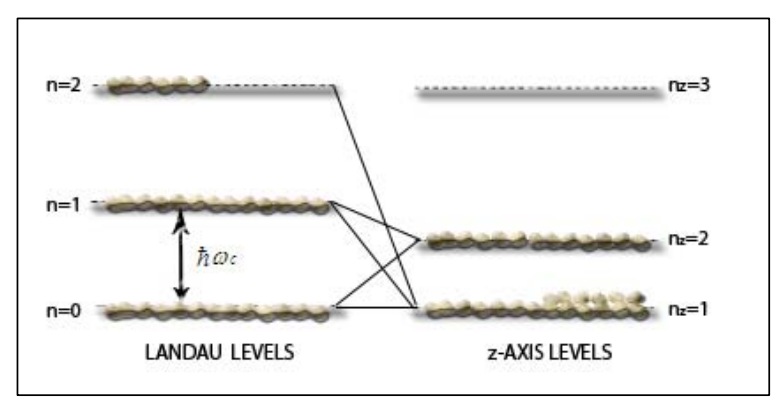

The equality $\Delta \mathrm{E}_{z}\{3,1\}=2 \hbar \omega_{c}$ is satisfied
Fig. 4.35: $d=\sqrt{\frac{5 \pi \Phi_{o}}{4 B}}$

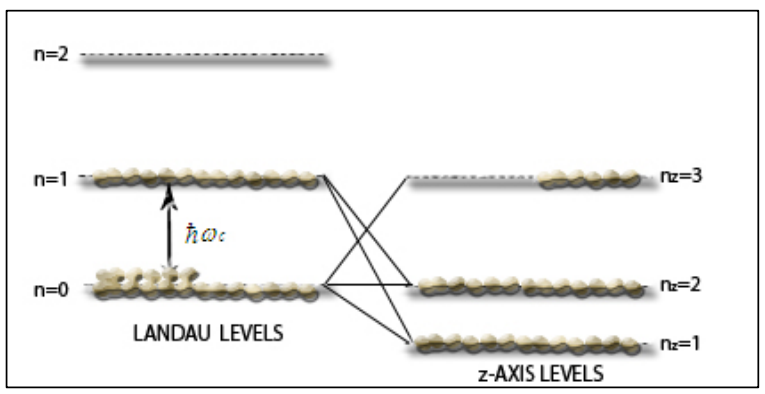

The equality $\Delta \mathrm{E}_{z}\{3,2\}=\hbar \omega_{c}$ is satisfied. 
Fig. 4.36: $d \geq \sqrt{\frac{5 \pi \Phi_{o}}{4 B}}$

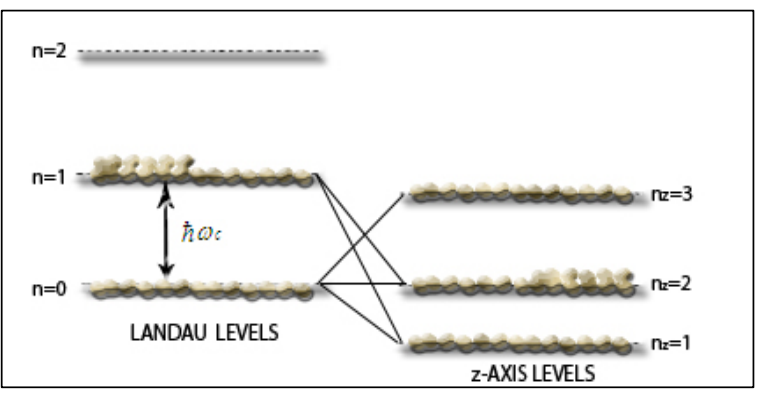

States $\{n=0, n z=3\}$ are fully occupied, while $\left\{n=1, n_{z}=2\right\}$ are now partially filled because $\Delta \mathrm{E}_{z}\{3,2\}<\hbar \omega_{c}$

Fig. 4.38: $d \geq \sqrt{\frac{8 \pi \Phi_{o}}{4 B}}$

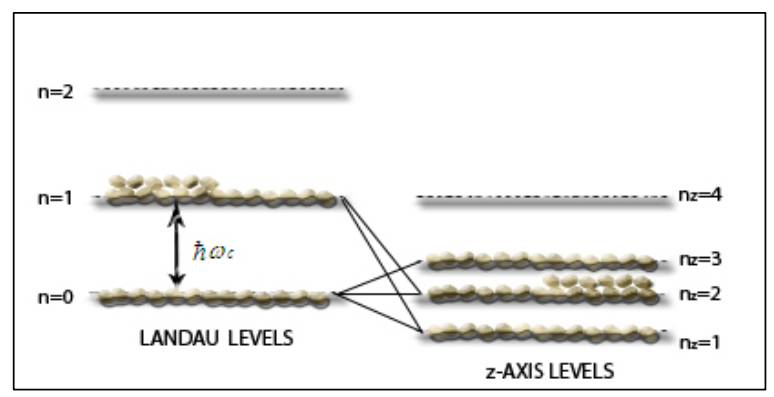

No further changes

Fig. 4.40: $d \geq \sqrt{\frac{12 \pi \Phi_{o}}{4 B}}$

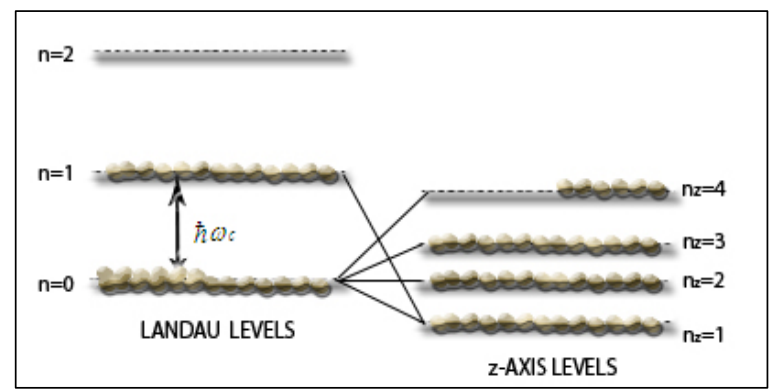

States $\left\{n=1, n_{z}=2\right\}$ are abandoned and $\left\{n=0, n_{z}=4\right\}$ are partially occupied because $\Delta \mathrm{E}_{z}\{4,2\}<\hbar \omega_{c}$
Fig. 4.37: $d=\sqrt{\frac{8 \pi \Phi_{o}}{4 B}}$

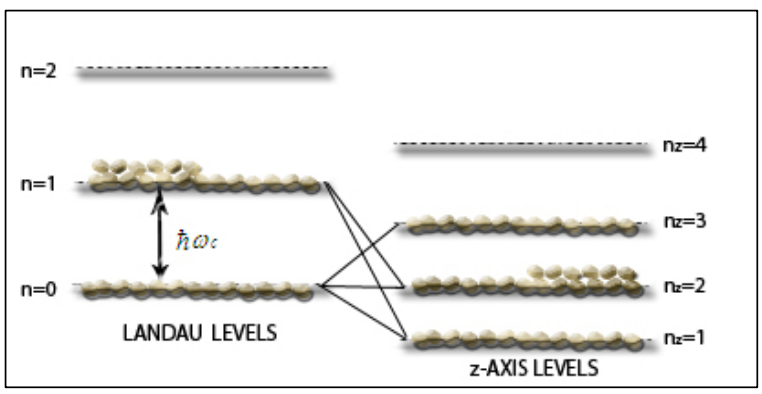

No further changes

Fig. 4.39: $d=\sqrt{\frac{12 \pi \Phi_{o}}{4 B}}$

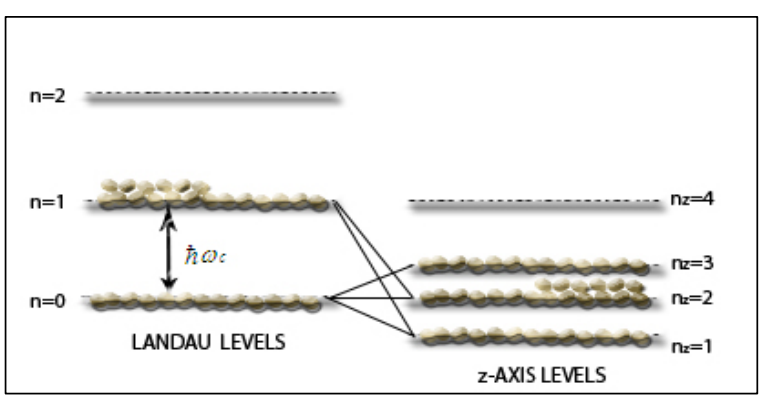

The equality $\Delta \mathrm{E}_{z}\{4,2\}=\hbar \omega_{c}$ is satisfied

Fig. 4.41: $d=\sqrt{\frac{15 \pi \Phi_{o}}{4 B}}$

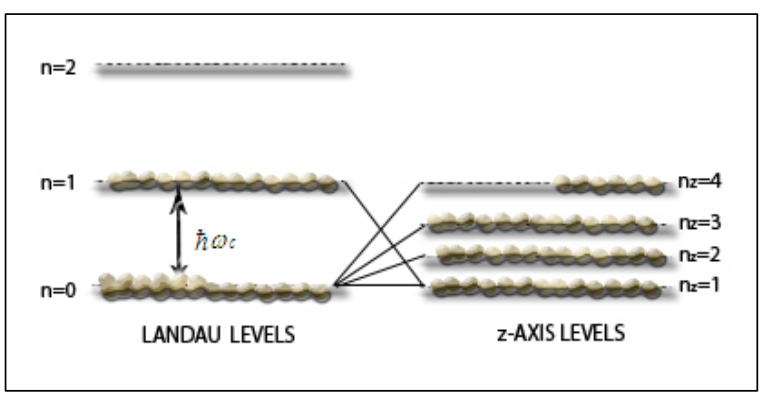

The equality $\Delta \mathrm{E}_{z}\{4,1\}=\hbar \omega_{c}$ is satisfied 
Fig. 4.42: $d \geq \sqrt{\frac{15 \pi \Phi_{o}}{4 B}}$

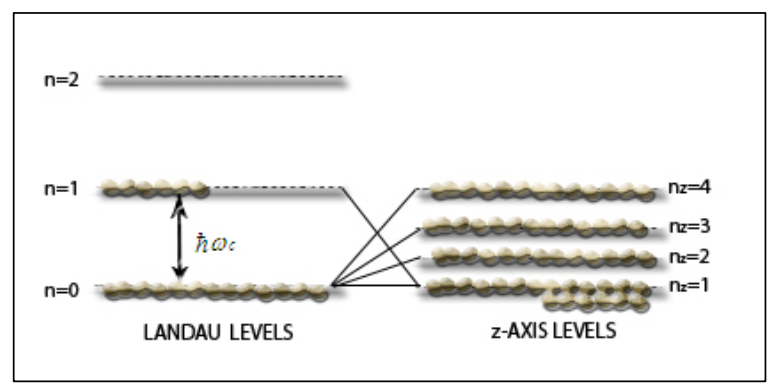

States $\{n=0, n z=4\}$ are fully occupied, while $\{n=1, n z=1\}$ are partially filled because $\Delta \mathrm{E}_{z}\{4,1\}<\hbar \omega_{c}$
Fig. 4.43: $d=\sqrt{\frac{24 \pi \Phi_{o}}{4 B}}$

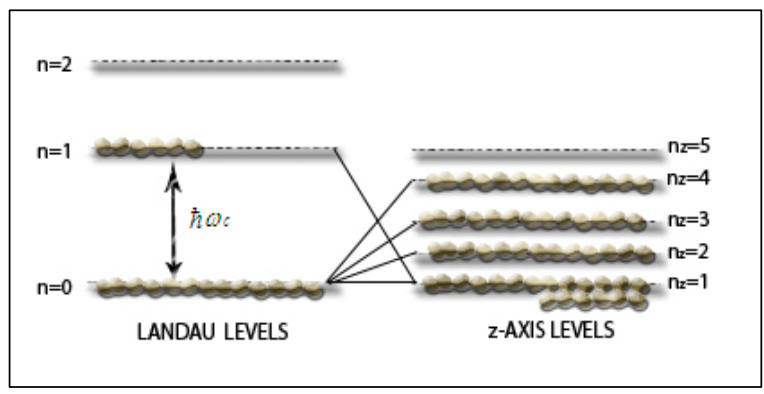

The equality $\Delta \mathrm{E}_{z}\{5,1\}=\hbar \omega_{c}$ is satisfied

Fig. 4.44: $d \geq \sqrt{\frac{24 \pi \Phi_{o}}{4 B}}$

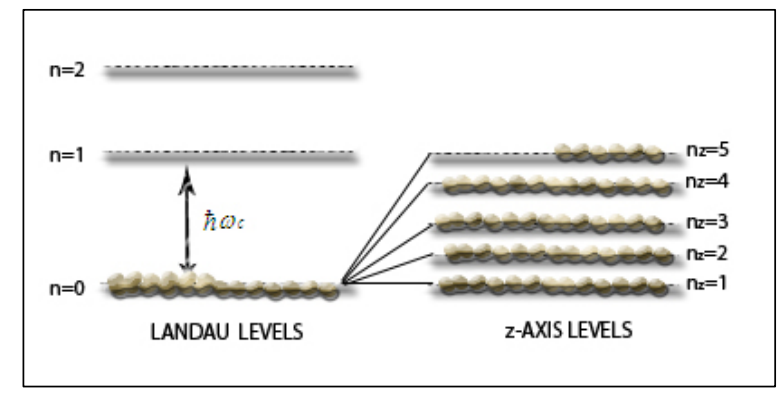

All distinct $z$-axis levels are occupied, combined with the lowest LL, because $\Delta \mathrm{E}_{z}\{5,1\}<\hbar \omega_{c}$

From these examples we can observe some well-defined patterns at the two ends of the procedure, i.e., of the range of variation of $B$ and $d$. However, we also observe a certain unpredictability that requires utmost care in the middle of the procedure. For example, note that in Figs. 4.35-4.40, comparisons have to involve higher QW levels. After the optimal scenarios are carefully found and run, for every window of $B$ and $d$ values, it is straightforward to write down analytically the total energy for each case. Then, the most important information that remains is to draw the graphs of the total energy, magnetisation and susceptibility as functions of the thickness $d$, or magnetic field $B$, or both. Once again, although fixed $B$-variations describe better the theoretical patterns, fixed $d$ is the experimentally relevant case, the combined variation also being provided in $2 \mathrm{D}$ graphs later, which demonstrates everything in a compact manner. In some of the figures below, we take the areal density to be $n_{A}=10^{16} \mathrm{~m}^{-2}$. We first plot the $1 \mathrm{D}$ graphs, i.e., with respect to one variable only and the $2^{\text {nd }}$ held fixed. Later, we present some 2D graphs under combined variations of $B$ and $d$. First, we keep $B$ fixed and the reader should recall that, although the thickness $d$ is treated as an independent variable, the windows of $d$-values, for which we have a particular analytical expression for the total energy $\mathrm{E}$, do depend on $B$. 


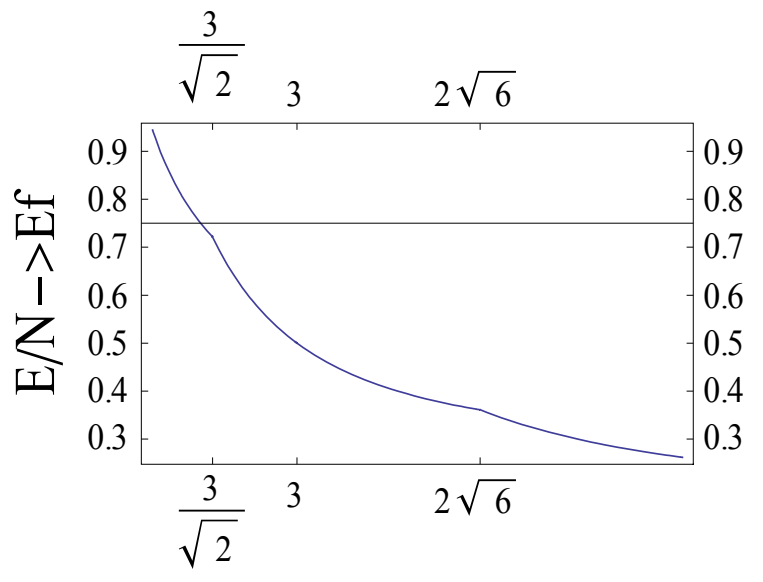

$\mathrm{d}->\sqrt{\pi / 2 n_{A}}$

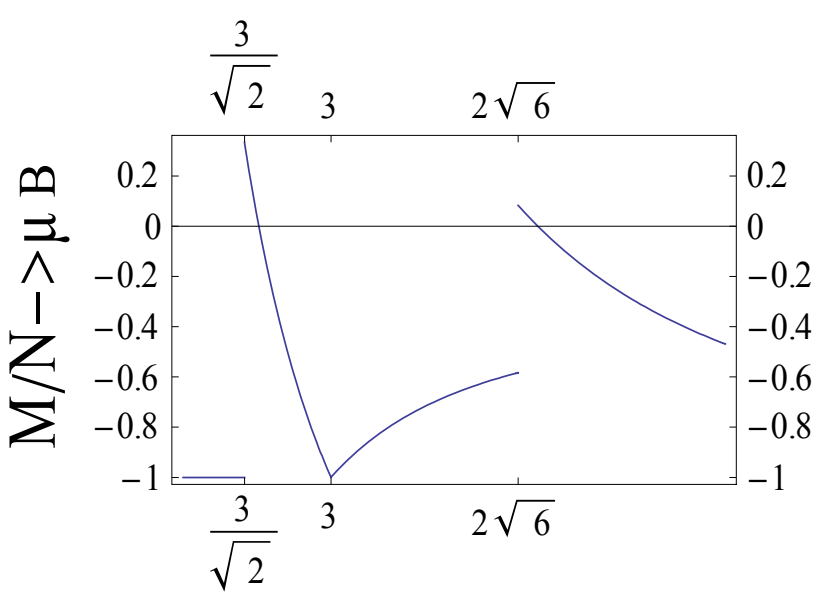

$\mathrm{d}->\sqrt{\pi / 2 n_{A}}$

[A]

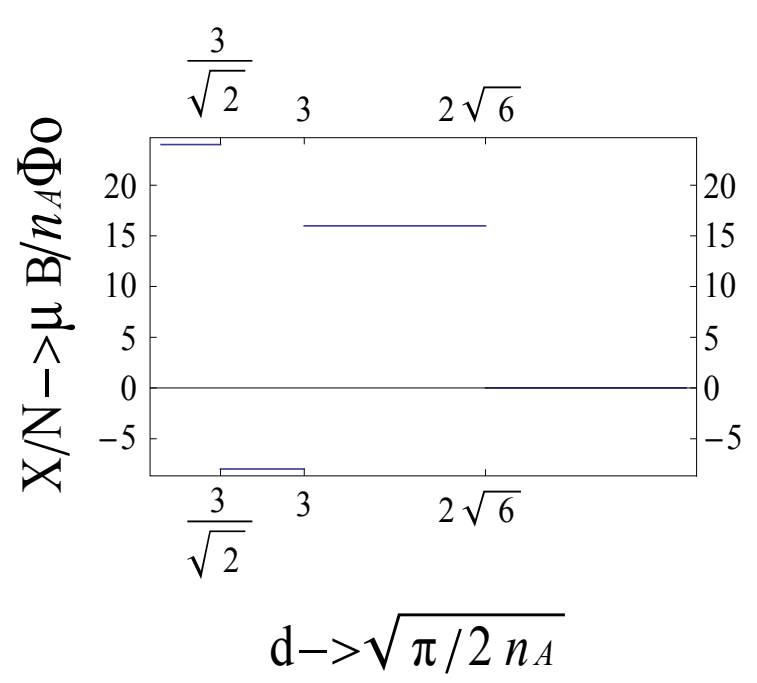

[B]

[C]

Fig. 4.45: Graphs: A) Energy, B) Magnetisation and C) Susceptibility per electron as functions of thickness $d$ when the magnetic field is $1 / 6 \mathrm{n}_{\mathrm{A}} \Phi_{\mathrm{o}}$ (hence, we have complete LL filling). Note that susceptibility can be negative (as opposed to the $2 \mathrm{D}$ case). 

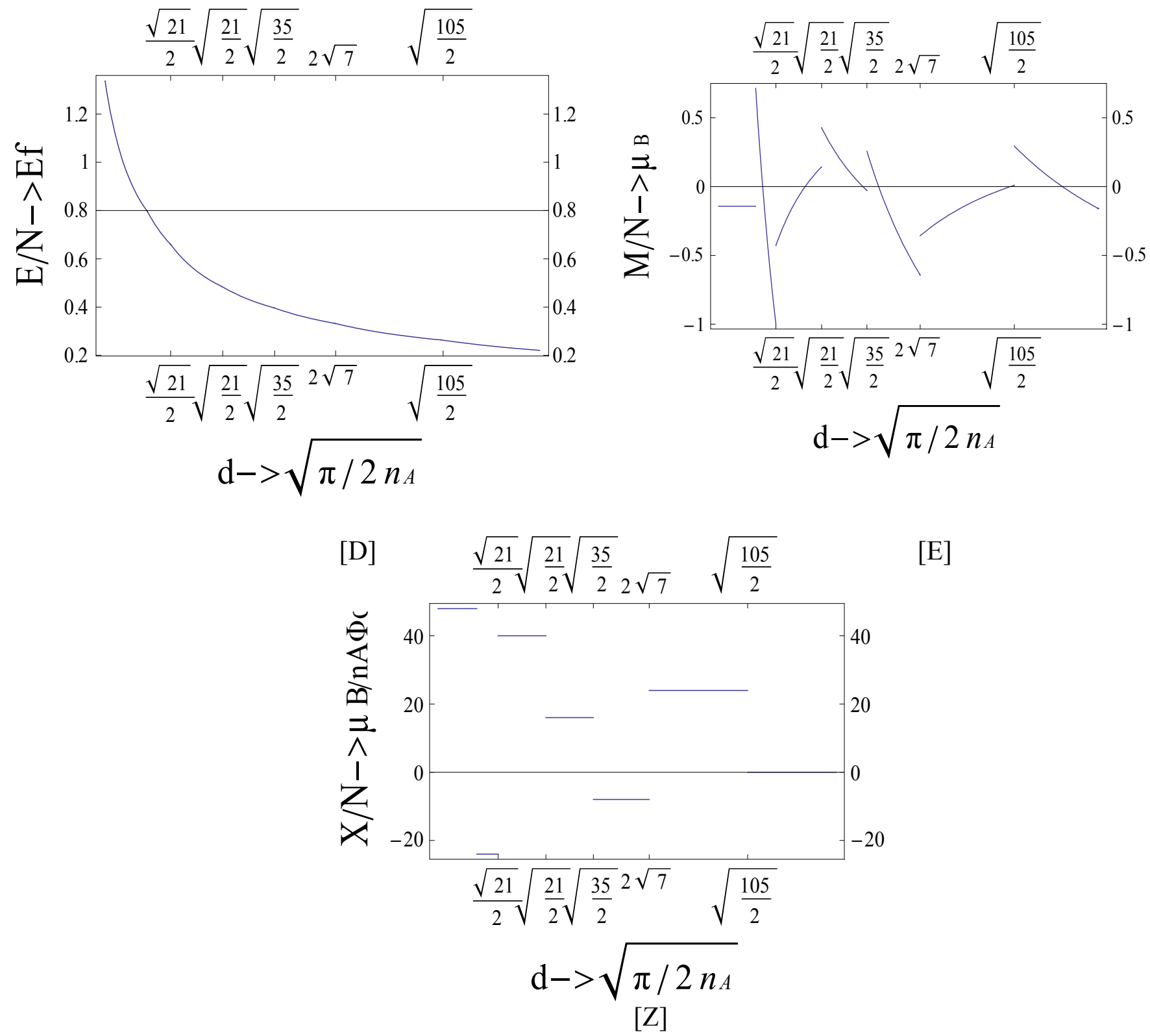

[E]

. 4.46: Graphs: D) Energy, E) Magnetisation and Z) Susceptibility per electron as functions of thickness when the magnetic field is $1 / 7 \mathrm{n}_{A} \Phi_{\mathrm{o}}$ (hence, we now have partial LL filling). Although the total energy is everywhere continuous, note the discontinuities that take place in the magnetisation and magnetic susceptibility; the latter might have negative values, contrary to the $2 \mathrm{D}$ system that only gave positive values. It should be noted that all transitions shown here are "internal transitions" and that this affects later figures when $B$ will be varied (for fixed $d$ ), where each of these transitions will appear as "internal breakings".

We should note again that the new (internal) transitions found above correspond to incomplete LL filling and one could be tempted to speculate that these might lead to interesting effects, pertinent to fractional fillings and the FQHE, if interactions were included, or even turned on perturbatively. However, let us make the choice to restrict ourselves to noninteracting particles for consistency of the approach. After all, ultimately, we want to apply this line of reasoning to topological insulators (see Section 6), which are actually defined in a one-electron Physics picture.

Next, we present again some 1D graphs but for the case where the thickness $d$ is kept constant and magnetic field $B$ is varied. Note the discontinuities in magnetisation and magnetic susceptibility for some values of the magnetic field $B$. Furthermore, there are cases where magnetisation might also have discontinuities in the interior of a $B$-window, see, graph $[\mathrm{M}]$ at a value of $1 / B=15 / n_{A} \Phi_{0}$, which is an example of an "internal breaking"; such breakings, which are actually phase transitions corresponding to partial LL filling, as we saw earlier, have not been noted in theoretical treatments in the past and they are not in accordance with the dHvA effect. 
Thermodynamic quantities for $d=\sqrt{\frac{30 \pi}{n_{A}}}$

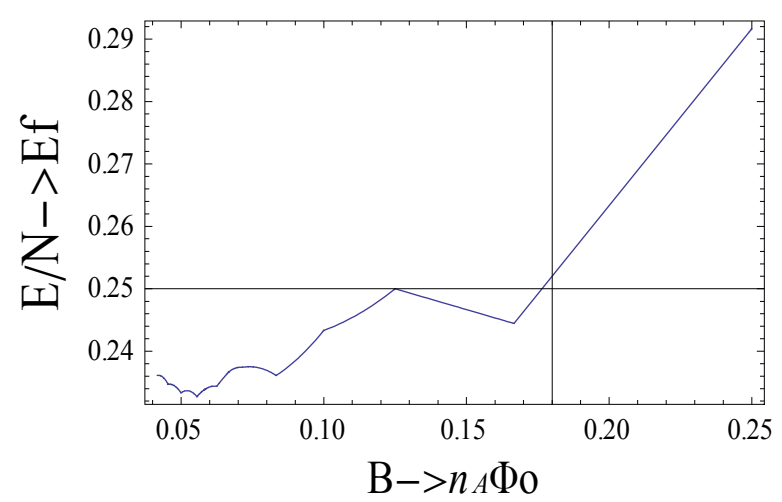

[I]

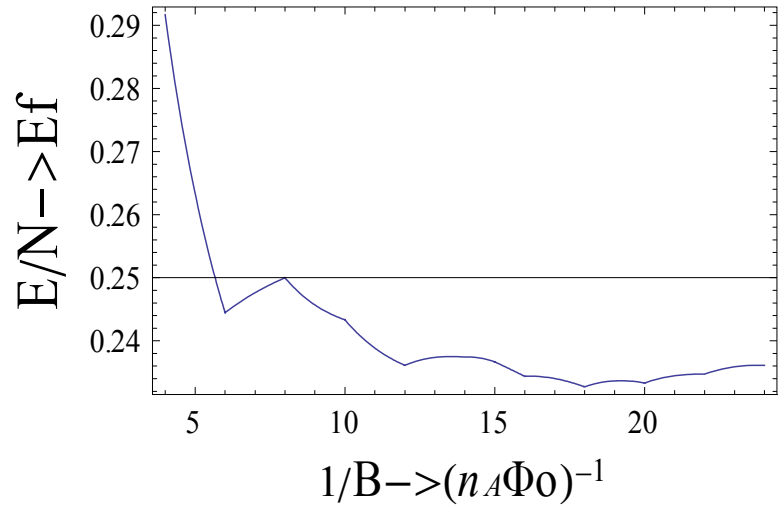

[K]
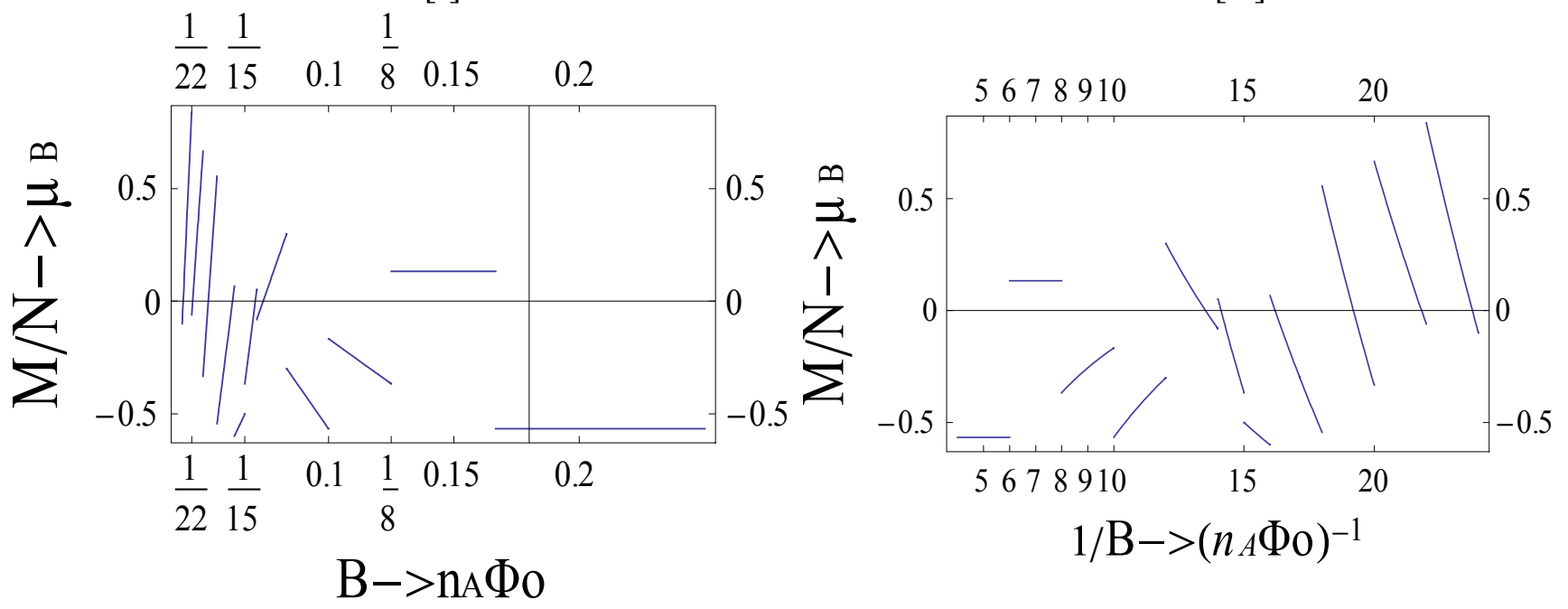

[L]

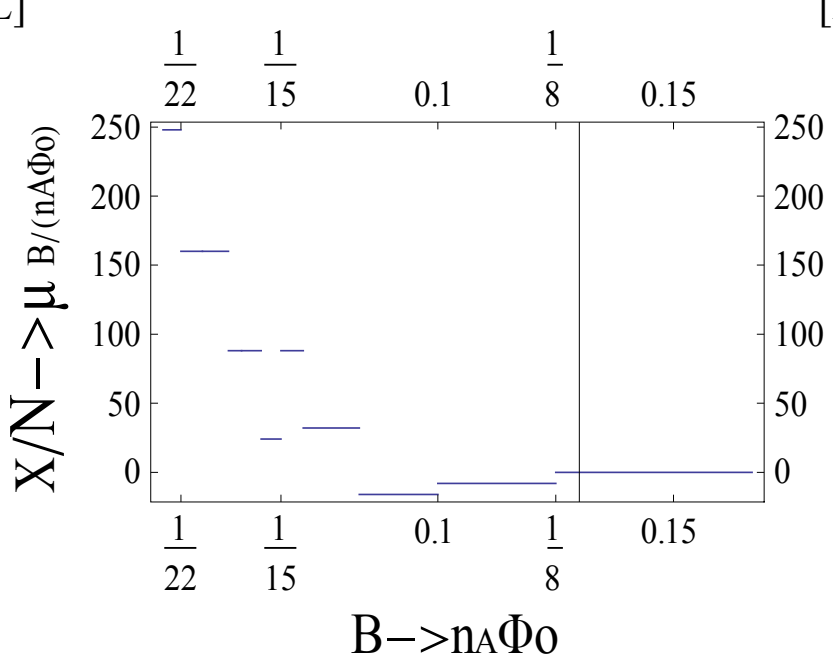

[N]

Fig. 4.47: Graphs: I) Energy and L) Magnetisation and N) Susceptibility per electron as functions of $B$ and K) Energy, M) Magnetisation as functions of inverse $B$. 
Thermodynamic quantities for: $d=\sqrt{\frac{375 \pi}{2 n_{A}}}(242 \mathrm{~nm})$

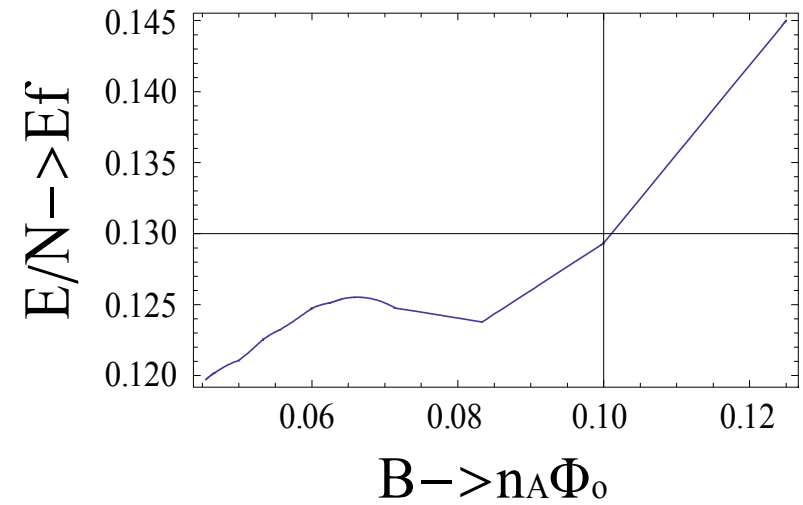

$[\mathrm{O}]$

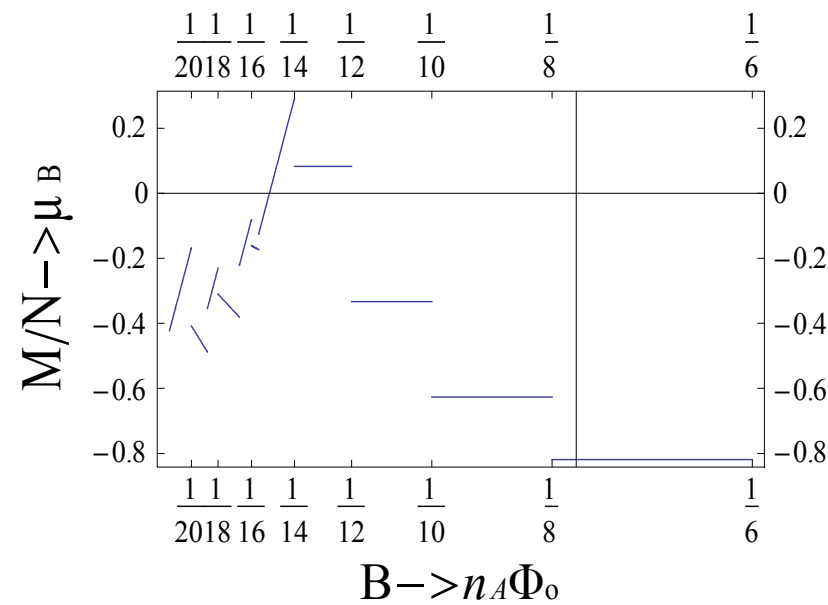

$[\mathrm{P}]$

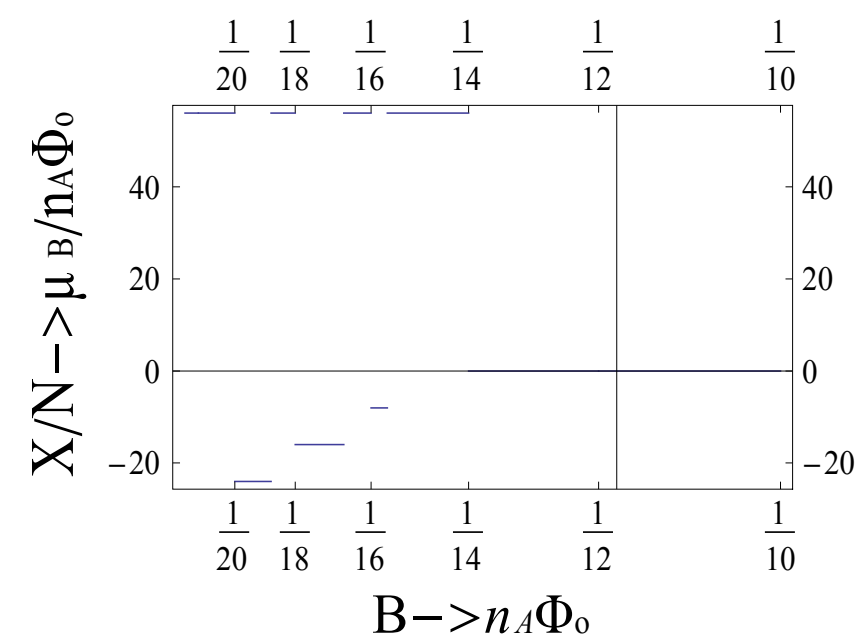

[Q]

Fig. 4.48: Graphs: O) Energy P) Magnetisation and Q) Susceptibility per electron as functions of $B$. (Note internal transitions at $1 / \mathrm{B} \sim 15.5$ and also $\sim 18.8$ in units of $1 / n_{A} \Phi_{0}$.)

Some comments concerning these graphs are now in order. Energy is always (as in the case of 2D) a continuous function of the magnetic field, as expected on general physical grounds. Graph $\mathrm{O}$ shows the energy as a function of $B$ for a somewhat large thickness (about $242 \mathrm{~nm}$ for an areal density about $10^{16} \mathrm{~m}^{-2}$ ), which as we shall see in the next section, looks almost identical in numerical values to the energy that comes out for the case of full 3D space with periodic boundary conditions (see Section 5). Although, we will see that the energy for that system is perfectly smooth (continuous and differentiable), while here it still has cusps, i.e., the magnetization has discontinuities. All thermodynamic quantities, such as energy, magnetisation and susceptibility converge to the corresponding full three-dimensional quantities when the thickness is very large, signifying that boundary conditions (here, a double rigid wall) do not actually matter when the space available to electrons is very large, at least for this conventional system.

While energy is a continuous function of $B$, magnetisation and susceptibility on the other hand, are not. With respect to the critical values of $B$, where all energy states are fully occupied (or fully empty), this is not a surprise. We could predict these discontinuities by examining the semi-classical dHvA effect, according to which magnetisation and susceptibility are periodic functions of $1 / B$ with period $2 / n_{A} \Phi_{o}$. However, these are not the only types of discontinuities here; from graphs $[\mathrm{P}]$ and $[\mathrm{Q}]$, one notices that there are cases where magnetisation and susceptibility have discontinuities, even in the interior of a dHvA window of $B$-values (see, 
for example, graph $\mathrm{P}$ and $\mathrm{Q}$ at values of $1 / \mathrm{B} \sim 15.5$ and 18.8 in units of $1 / n_{A} \Phi_{0}$ ). This is a new observation; a result not captured by other approaches and one that demonstrates the nontrivial role that thickness $d$ plays, even in this simple problem.

In the following, we also present the corresponding 3D graphs of all thermodynamic properties, as functions of combined variations of both $B$ and $d$, for the first few windows of $B$-values.

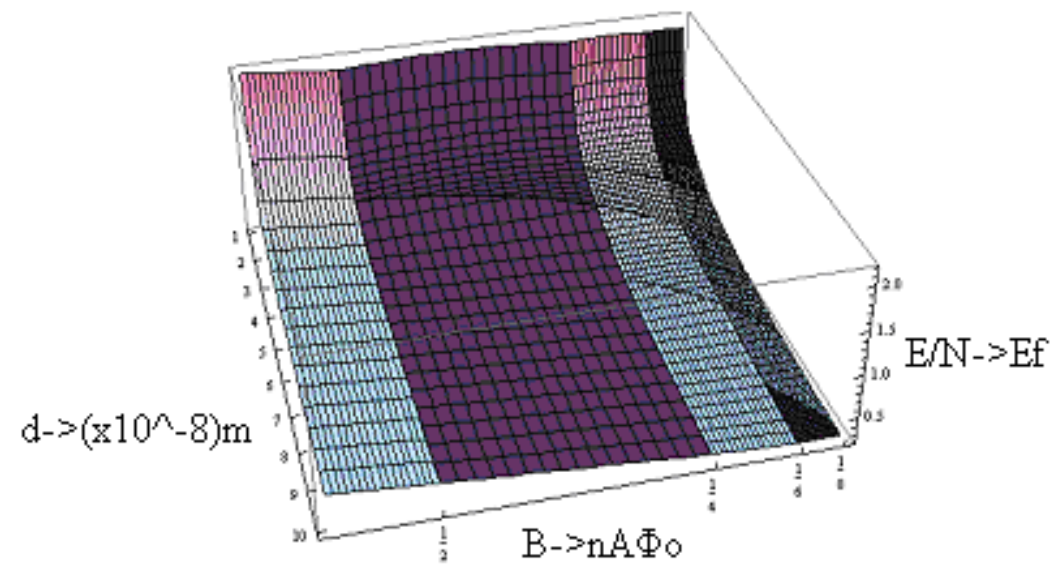

Fig. 4.49: Total energy as a function of both $B$ and $d$

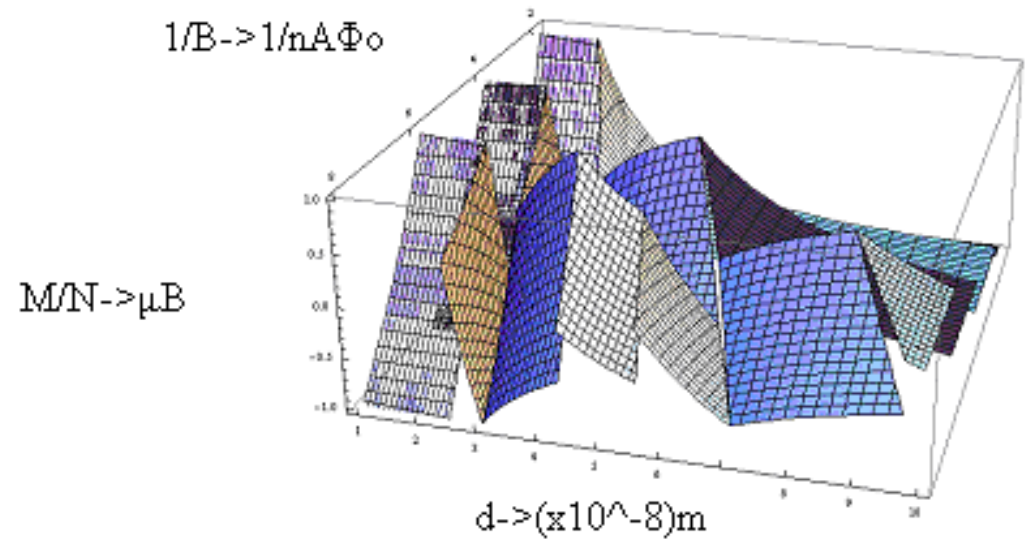

Fig. 4.50: Magnetisation as a function of both $B$ and $d$. 


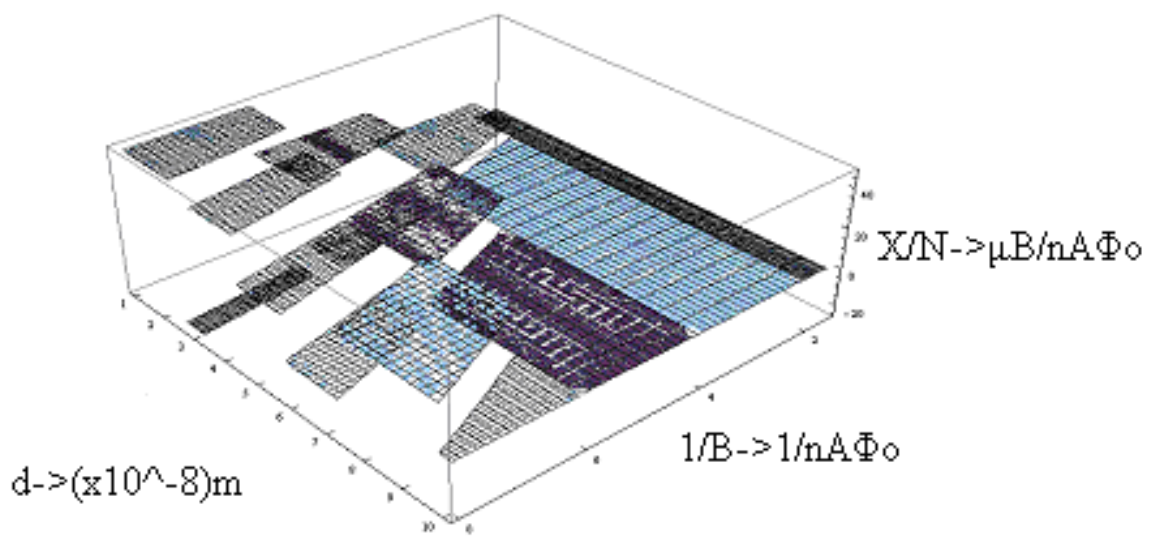

Fig. 4.51: Magnetic susceptibility as a function of $B$ and $d$.

Let us summarise some observations concerning all these results. Unlike the energy, magnetisation and susceptibility are strongly discontinuous, both in the $B$ - and $d$-axis; one should notice the oscillations along the $B$-axis when thickness is very small, where the system behaves effectively as being two-dimensional. These rapid discontinuities create, for a very thin film, a sawtooth behaviour similar to the case of two dimensions. We conclude that the system oscillates between paramagnetism and diamagnetism; hence, it experiences phase transitions, because magnetisation also changes its slope during the increase of $B$ or $d$. As thickness increases, the single-particle energetic configurations change in a manner that is not predictable a priori and this has consequences. There are new transitions occurring, qualitatively different from $2 \mathrm{D}$; magnetisation can be discontinuous, even in the interior of a $B$-window when the highest LL is incompletely filled, which is something that violates the standard periodicities given by the dHvA effect that are always related to complete filling in 2D. This arises from the energy interplay between the LL and QW levels and as already emphasised, it occurs in patterns that are not easily predictable. These patterns are quite esoteric and we are omitting a detailed discussion but we can quickly give some further quantitative observations; magnetisation and susceptibility may have several discontinuities for arbitrary values of $B$ and $d$ and we have noted that in each $B$-window there are exactly $\rho(\rho-1) / 2+1 d$-windows with $\rho$ being the total number of combined states involved. This means that magnetisation and susceptibility have $\rho(\rho-1) / 2$ discontinuities inside that window. For example, if $B$ is equal to $\left(n_{A} \Phi_{o}\right) / 5$, then $\rho=3$ and magnetisation will have discontinuities at 24, 34 and $56 \mathrm{~nm}$.

\section{Relation to transport properties - Hall conductivity}

The usual criterion for the existence of IQHE is that the Fermi energy must lie in a bulk energy gap, which is actually the well-known mobility gap created by disorder and then chiral currents flow along the edges. This picture is valid for a planar two-dimensional system, where no freedom in the $z$-axis is present. One then wonders how this picture is modified in the case of our interface. How are the diamagnetic chiral currents generalised in the presence of a strongly quantised $z$-direction? A first thought is that these one-dimensional current channels now become surface currents that move in opposite directions in the two opposite edge-surfaces of the interface. This may not be a bad picture, because from the energy spectrum (4.1), one notes that there is in fact no dispersion at all; the net velocity in the $z$-axis is zero. Including a confining potential in the $x$-direction, surface diamagnetic currents are then created in two edge surfaces, while the net velocity in the bulk of the system vanishes, i.e., the net current is then zero. For a nonzero surface current, one must shift the electrochemical potentials of the edges by a moderate amount and this is achieved directly by applying a small in-plane electric field, which controls the number of edge surface states.

Now let us consider a clean sample and see when the Fermi energy lies in a gap. This condition is only met whenever the magnetic field is exactly of the form $1 / 2 \rho n_{A} \Phi_{o}$, in the sense that there are no infinitesimally close neighbouring empty states for the electron to be scattered in. We expect that at these special values of $B$, where all LLs are fully occupied, the Hall conductivity will be quantised as in the usual 2D case in units of $e^{2} / h$ for 
every value of thickness $d$. This actually results from a semi-classical treatment of the problem, where the Hall conductivity is of the form:

$$
\sigma_{\mathrm{H}}=\frac{n_{V} e c}{B}=\frac{n_{A} e c}{d B}
$$

where $n_{V}=n_{A} / d$ is the average volume density. Substituting the special window values of $B$ (namely $\left.B=\frac{1}{2 \rho} n_{A} \Phi_{o}\right)$ in (4.26), we have:

$$
\sigma_{\mathrm{H}}=\frac{2 \rho e^{2}}{h d}
$$

which is a result that may apply to multi-layered QHE systems [7]. An alternative way to obtain the above result is to use the analogue of the mathematical relation (2.10) introduced in Section 2, which relates the discontinuities of orbital magnetisation with the corresponding discontinuities of chemical potential at the critical values of $B$, namely:

$$
\sigma_{\mathrm{H}}=\frac{e c \Delta \mathrm{M}}{d \Delta \mu S}
$$

Let us use (4.28) in an example in order to check the validity of (4.27) when $B=(1 / 2 \rho) n_{A} \Phi_{o}$, namely, when $\rho$ sets of combined states are fully occupied. Then, the criterion of quantisation of conductivity is fulfilled, because Fermi energy is in a gap. If $\rho=1$, i.e., only one combined state is occupied, then from (4.8) we have for the energy:

$$
\frac{E_{1}}{N}=E_{f}\left[\frac{B}{n_{A} \Phi_{o}}+\left(\frac{\pi}{2 n_{A} d^{2}}\right)\right], \text { valid for every } d
$$

and from (4.16)

$$
\frac{E_{2}}{N}=E_{f}\left[\left(\frac{B}{n_{A} \Phi_{o}}\right)-6\left(\frac{B}{n_{A} \Phi_{o}}\right)\left(\frac{\pi}{2 n_{A} d^{2}}\right)+\left(\frac{4 \pi}{2 n_{A} d^{2}}\right)\right], \text { valid for } d \geq \sqrt{\frac{3 \pi \Phi_{o}}{4 B}}=\sqrt{\frac{3 \pi}{2 n_{A}}} .
$$

The corresponding magnetisations are for this case:

$$
\begin{gathered}
\frac{M_{1}}{N}=-\mu_{B}, \\
\frac{M_{2}}{N}=\mu_{B}\left[-1+6\left(\frac{\pi}{2 n_{A} d^{2}}\right)\right],
\end{gathered}
$$

with $\Delta M=M_{2}-M_{1}=6 N \mu_{B}\left(\frac{\pi}{2 n_{A} d^{2}}\right)$.

The chemical potentials are (compare highest single-particle energies in Figs. 4.1 and 4.3)

$$
\begin{aligned}
& \mu_{1}=\frac{\hbar \omega_{c}}{2}+\frac{\hbar^{2} \pi^{2}}{2 m d^{2}} \\
& \mu_{2}=\frac{\hbar \omega_{c}}{2}+\frac{4 \hbar^{2} \pi^{2}}{2 m d^{2}}
\end{aligned}
$$

with $\Delta \mu=\mu_{2}-\mu_{1}=\frac{3 \hbar^{2} \pi^{2}}{2 m d^{2}}=3 \mu_{B} \Phi_{o} \frac{\pi}{2 d^{2}}$ 
By then applying (4.28), we get:

$$
\sigma_{\mathrm{H}}=\frac{e c \Delta \mathrm{M}}{d \Delta \mu S}=\frac{\operatorname{ec} 6 N \mu_{B}\left(\frac{\pi}{2 n_{A} d^{2}}\right)}{d 3 \mu_{B} \Phi_{o} \frac{\pi}{2 d^{2}} S}=2 \frac{e^{2}}{d h}
$$

in full accordance with (4.27) for $\rho=1$.

Another example is when $\rho=2$ and again, for complete filling, $B$ must be:

$$
B=\frac{1}{4} n_{A} \Phi_{o}
$$

Let us also consider the case when $d$ lies in the following window:

$$
d \leq \sqrt{\frac{3 \pi \Phi_{o}}{4 B}}=\sqrt{\frac{3 \pi}{n_{A}}}
$$

The discontinuity of $M$ is connected with the two neighbouring energies, one shown in (4.15) and the other could be, for example, the second expression in Table 3 (of course, the choice is made with respect to thickness, such that the two ranges match):

$$
\begin{gathered}
\frac{E_{1}}{N}=E_{f}\left[-4\left(\frac{B}{n_{A} \Phi_{o}}\right)^{2}+3\left(\frac{B}{n_{A} \Phi_{o}}\right)+\left(\frac{\pi}{2 n_{A} d^{2}}\right)\right] \\
\frac{E_{2}}{N}=E_{f}\left[4\left(\frac{B}{n_{A} \Phi_{o}}\right)^{2}+\left(\frac{B}{n_{A} \Phi_{o}}\right)-12\left(\frac{B}{n_{A} \Phi_{o}}\right)\left(\frac{\pi}{2 n_{A} d^{2}}\right)+\left(\frac{4 \pi}{2 n_{A} d^{2}}\right)\right] .
\end{gathered}
$$

The corresponding magnetisations are, respectively:

$$
\begin{gathered}
\frac{M_{1}}{N}=\mu_{B}\left[8\left(\frac{B}{n_{A} \Phi_{o}}\right)-3\right] \\
\frac{M_{2}}{N}=\mu_{B}\left[-8\left(\frac{B}{n_{A} \Phi_{o}}\right)-1+12\left(\frac{\pi}{2 n_{A} d^{2}}\right)\right] .
\end{gathered}
$$

So,

$$
\Delta \mathrm{M}=M_{2}-M_{1}=N \mu_{B}\left[-8\left(\frac{B}{n_{A} \Phi_{o}}\right)-1+12\left(\frac{\pi}{2 n_{A} d^{2}}\right)-8\left(\frac{B}{n_{A} \Phi_{o}}\right)+3\right]=N \mu_{B}\left[-16\left(\frac{B}{n_{A} \Phi_{o}}\right)+12\left(\frac{\pi}{2 n_{A} d^{2}}\right)+2\right] \text {. }
$$

For $B=\frac{1}{4} n_{A} \Phi_{o}$

$$
\Delta \mathrm{M}=N \mu_{B}\left[12\left(\frac{\pi}{2 n_{A} d^{2}}\right)-2\right]
$$

Now, we have for the chemical potentials (compare Figs. 4.2 and 4.6):

$$
\begin{gathered}
\mu_{1}=3 \frac{\hbar \omega_{c}}{2}+\frac{\hbar^{2} \pi^{2}}{2 m d^{2}} \\
\mu_{2}=\frac{\hbar \omega_{c}}{2}+\frac{4 \hbar^{2} \pi^{2}}{2 m d^{2}} \\
\Rightarrow \Delta \mu=\mu_{2}-\mu_{1}=\frac{\hbar \omega_{c}}{2}+\frac{4 \hbar^{2} \pi^{2}}{2 m d^{2}}-3 \frac{\hbar \omega_{c}}{2}-\frac{\hbar^{2} \pi^{2}}{2 m d^{2}}=-\hbar \omega_{c}+\frac{3 \hbar^{2} \pi^{2}}{2 m d^{2}},
\end{gathered}
$$




$$
\Delta \mu=-\frac{2 \hbar e B}{2 m c}+\frac{c}{e} \frac{3 \hbar^{2} \pi^{2} e}{2 m d^{2} c}=\mu_{B}\left(-2 B+\frac{3 \hbar \pi^{2} c}{d^{2} e}\right) \stackrel{B=\frac{1}{4} n A \Phi_{o}}{\longrightarrow}=\frac{\mu_{B} \Phi_{o} n_{A}}{4}\left(-2+\frac{12 \pi}{2 n_{A} d^{2}}\right)
$$

Substituting in (4.28), we have:

$$
\sigma_{\mathrm{H}}=\frac{e c \Delta \mathrm{M}}{d \Delta \mu S}=\frac{e c}{d S} \frac{N \mu_{B}\left[12\left(\frac{\pi}{2 n_{A} d^{2}}\right)-2\right]}{\frac{\mu_{B} \Phi_{o} n_{A}}{4}\left(-2+\frac{12 \pi}{2 n_{A} d^{2}}\right)}=4 \frac{e c N}{d S \Phi_{o} n_{A}}=4 \frac{e^{2}}{d h},
$$

which is in full accordance with (4.27) with $\rho=2$. If $B=(1 / 6) n_{A} \Phi_{o}$ we would then find $\sigma_{\mathrm{H}}=6 e^{2} / d h$, independently of the choice of thickness and so on. In conclusion, we see that if $B$ has the exact value necessary for the combined sets of degenerate states to be fully occupied, the transverse conductivity is quantised with universal values that are essentially the same as those of a 2D system, such as in a multi-layered QHE system [7]. The issue of the new transitions reported here for partial LL filling, requires as already noted, a closer investigation, because in this case we have the standard issue of the enormous degeneracy of the many-body states involved and to draw conclusions on transport one has to include electron-electron interactions; however, see Subsection 4.2 for a rather unconventional picture.

\subsection{Inclusion of Zeeman term}

When the gyromagnetic ratio $g^{*}$ is non-vanishing, the previous results will be modified and here, we give a quick discussion of the general manner in which the presence of $g^{*}$ is expected to affect them. By including the Zeeman term in our model, we have the following single-particle energy spectrum:

$$
\varepsilon_{n, k z}=\left(n+\frac{1}{2}\right) \hbar \omega_{c}^{*}+\frac{\hbar^{2} k_{z}^{2}}{2 m^{*}} \pm \frac{g^{*}}{2} \mu_{B} B
$$

where, for simplicity, $g^{*}$ is considered to be positive, $m^{*}$ is the electron's effective mass, $\mu_{B}=e \hbar / 2 m c$ is the Bohr magneton (with $m$ being the electron's vacuum mass) and $\omega_{c}{ }^{*}=e B / m^{*} c$ is the effective cyclotron frequency. The wavenumber $k_{z}$ is still quantised in the following manner; $k_{z}=\pi n_{z} / d$, with $n_{z}=1,2,3 \ldots$ We may write (4.1.1) in a more convenient form, namely:

$$
\varepsilon_{n, k z}=\left(n+\frac{1}{2} \pm \frac{g^{*}}{4} \frac{m^{*}}{m}\right) \hbar \omega_{c}^{*}+\frac{\hbar^{2} k_{z}^{2}}{2 m^{*}}
$$

This shows directly the well-known fact that, for the special case of noninteracting electrons in vacuo with $m^{*}=m$ and $g^{*}=2$, the Zeeman splitting is exactly equal to the LL splitting.

For the purposes of our calculation, we will confine $g^{*}$ in the range:

$$
0 \leq g^{*} \leq 2
$$

and will also assume $\mathrm{m}^{*}<\mathrm{m}$. The effect of Zeeman coupling is to split all Landau levels in two sublevels, where electrons are being placed according to their spin orientation, namely spin up and spin down (see Fig. 4.1.1). 


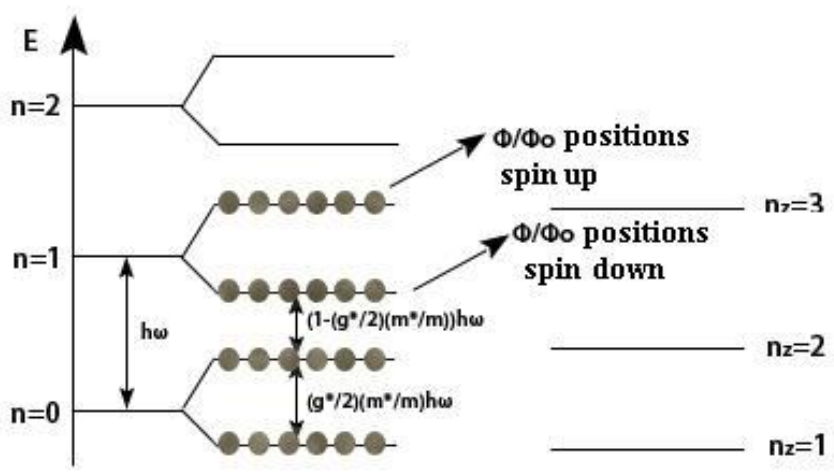

Fig. 4.1.1: Energetic configuration in the presence of Zeeman splitting (we have set $\omega=\omega_{c}$ )

We now have a different background structure for the possible energetic competitions. Firstly, the earlier degeneracy of each LL is partially lifted and each Zeeman sublevel contains $\Phi / \Phi$ independent states that can only accommodate electrons of a single spin. Secondly, gaps between different sublevels appear, which depend on the gyromagnetic ratio and on the effective mass, while the original inter-LL gaps are still present and equal to $\hbar \omega_{c}$. Thirdly, if we happen to have $m^{*}=m$ and $g^{*}=2$, then Zeeman splitting coincides with LL spacing and nearby sublevels fall on top of each other; therefore, doubling their degeneracy to $2 \Phi / \Phi o$ as before (except the lowest zero-energy state that remains with a degeneracy $\Phi / \Phi$ ). In what follows, we will denote each set of degenerate quantum states with

$$
\left\{(n, X), n_{z}\right\}, \quad \text { where } \mathrm{X}=\uparrow \text { or } \downarrow
$$

with a fourth quantum number $l$ (that counts each sublevel degeneracy) omitted, because as earlier, this will naturally be accounted for in the occupation procedure.

Let us now examine the first $B$-window that naturally comes up for this problem, namely:

$$
B \geq n_{A} \Phi_{o},
$$

where $n_{A}=N / S$ is always the constant areal density. It is now clear that for such a $B$, only the lowest sublevel is occupied (combined with $n_{z}=1$ ), namely $\left\{n=0 \downarrow, n_{z}=1\right\}$, with total energy given by:

$$
E=N \varepsilon\left\{n=0 \downarrow, n_{z}=1\right\},
$$

or in units of the effective Fermi energy:

$$
\frac{E}{N}=E_{f}^{*}\left[\frac{B}{n_{A} \Phi_{o}}\left(1-\frac{g^{*}}{2} \frac{m^{*}}{m}\right)+\frac{\pi}{2 n_{A} d^{2}}\right] .
$$

The above result describes a completely polarised state, where all spins are parallel in a direction opposite to $B$. For $g^{*}=0$, it coincides with (4.8), as it should, whereas for $g^{*}=2$ and $m^{*}=m$ there remains only the $z$-term, owing to the zero-energy of planar motion in this case. 


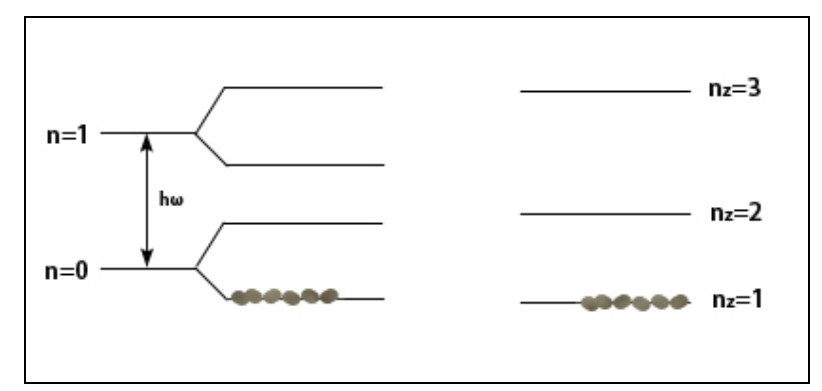

Fig. 4.1.2: When $B$ lies in the first window, all electrons fall into a completely polarised state

This first $B$-window leads to total energy linear in $B$. Let us now lower $B$, such that we lie in the second $B$ window, which is:

$$
\frac{1}{2} n_{A} \Phi_{o} \leq B \leq n_{A} \Phi_{o} .
$$

Here, due to Pauli's principle, we are forced to accommodate the extra $N-\Phi / \Phi o$ electrons into another sublevel. This requires care because we have to take into account the finite thickness $d$, which will decide for us the proper occupation scenario. We have three options: we can just change the LL index and move to $n=2$, or we can change the Zeeman sublevel and so reverse $N-\Phi / \Phi o$ electrons' spin, or we can change only the QW level and restart with the lowest possible values of all the remaining quantum numbers. Let us examine which option is the favourite.

At first, we should immediately note that changing the LL index would cost more energy than changing sublevel (reversing spins). Therefore, we are really left with just two options. The choice between them is thicknessdependent. It can be made by examining when the two remaining options (for an extra single electron) become equal in energy, which will immediately determine the transition between the two scenarios, namely:

$$
\begin{gathered}
\varepsilon \underbrace{\left\{n=0 \uparrow, n_{z}=1\right\}}_{\text {Change spin sublevel }}=\varepsilon \underbrace{\left.\varepsilon n=0 \downarrow, n_{z}=2\right\}}_{\text {Change QW sublevel }} \\
\left(\frac{1}{2}+\frac{g^{*}}{4} \frac{m^{*}}{m}\right) \hbar \omega_{c}^{*}+\frac{\hbar^{2} \pi^{2}}{2 m^{*} d^{2}}=\left(\frac{1}{2}-\frac{g^{*}}{4} \frac{m^{*}}{m}\right) \hbar \omega_{c}^{*}+\frac{4 \hbar^{2} \pi^{2}}{2 m^{*} d^{2}} \Rightarrow d_{\text {crit }}=\sqrt{\frac{3 \pi \Phi_{o}}{2 g^{*} B} \frac{m}{m^{*}}} .
\end{gathered}
$$

From this we can infer the following; when the thickness $d$ is lower than (4.1.7), namely, when the QW gaps are large enough, it is favourable to place the extra electron in the next available spin-up sublevel that lies in the $n=0 \mathrm{LL}$ and keep it in the QW level $n z=1$. When the thickness is larger than (4.1.7), it must go to $n_{z}=2$ by keeping its spin down in the same sublevel without violating Pauli's principle (see Figs. 4.1.3 and 4.1.4). If we substitute in (4.1.7) the largest value of $B$ (i.e., $B=n_{A} \Phi_{o}$ ), then we find a new criterion for 2-dimensionality (for $\left.d \leq d_{\min }\right)$, which depends strongly on $g^{*}$ :

$$
d_{\min }=\sqrt{\frac{3 \pi}{2 n_{A}}\left(\frac{m}{m^{*} g^{*}}\right)} .
$$

This is of course different from (3.6). Note that if we set $g^{*}=0$, it tends to infinity because it describes spinrelated Physics (we are always in the lowest LL, unlike the situation of the previous subsection). Let us then determine the new energies:

$$
\begin{aligned}
E & =\frac{\Phi}{\Phi_{o}} \varepsilon\left\{n=0 \downarrow, n_{z}=1\right\}+\left(N-\frac{\Phi}{\Phi_{o}}\right) \varepsilon\left\{n=0 \uparrow, n_{z}=1\right\} \\
& \Rightarrow \quad \frac{E}{N}=E_{f}^{*}\left[-g^{*} \frac{m^{*}}{m}\left(\frac{B}{n_{A} \Phi_{o}}\right)^{2}+\frac{B}{n_{A} \Phi_{o}}\left(1+\frac{g^{*}}{2} \frac{m^{*}}{m}\right)+\frac{\pi}{2 n_{A} d^{2}}\right], d \leq d_{\text {crit }}
\end{aligned}
$$




$$
\begin{aligned}
& E=\frac{\Phi}{\Phi_{o}} \varepsilon\left\{n=0 \downarrow, n_{z}=1\right\}+\left(N-\frac{\Phi}{\Phi_{o}}\right) \varepsilon\left\{n=0 \downarrow, n_{z}=2\right\} \\
& \Rightarrow \quad \frac{E}{N}=E_{f}^{*}\left[\frac{B}{n_{A} \Phi_{o}}\left(1-\frac{g^{*}}{2} \frac{m^{*}}{m}\right)-3\left(\frac{B}{n_{A} \Phi_{o}}\right)\left(\frac{\pi}{2 n_{A} d^{2}}\right)+\frac{4 \pi}{2 n_{A} d^{2}}\right], \quad d \geq d_{\text {crit }} .
\end{aligned}
$$

Note that if we set $g^{*}=0$ and $m^{*}=m$ we get:

$$
\frac{E}{N}=E_{f}\left[\frac{B}{n_{A} \Phi_{o}}+\frac{\pi}{2 n_{A} d^{2}}\right] \text { valid for any } d,
$$

which coincides with (4.8), as it should.

Fig. 4.1.3: $d \leq d_{\text {crit }}$

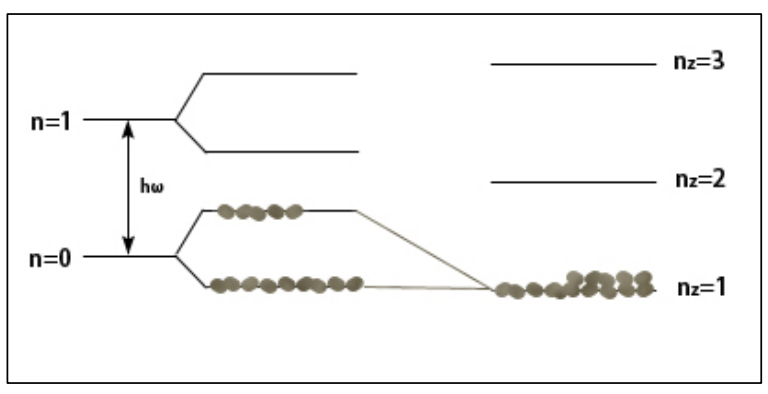

Fig 4.1.4: $d \geq d_{\text {crit }}$

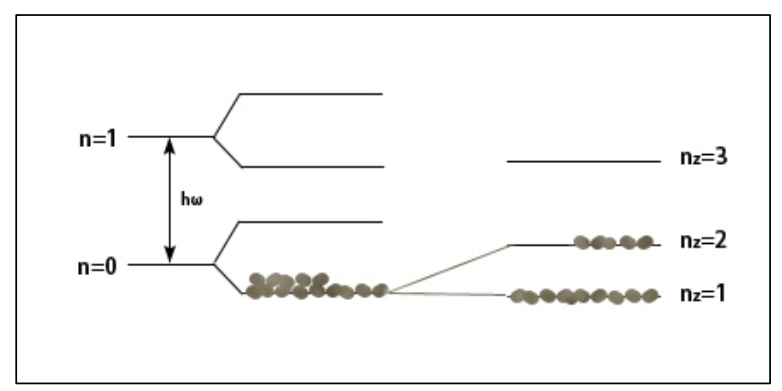

Following a similar line of reasoning for all $B$-windows, we can find all energetically optimal configurations that are now richer in transitions compared with the ones in the previous subsection but we will not show any further examples. In the following, we will first present one-dimensional figures based on the above example, as well as the corresponding $2 \mathrm{D}$ ones with combined variations of variables. The reader may compare them with those of the previous subsection in order to assess the differences.

In the figures, we always use the following values of $g^{*}, m^{*}$ :

$$
g^{*}=0.8, m^{*}=m
$$

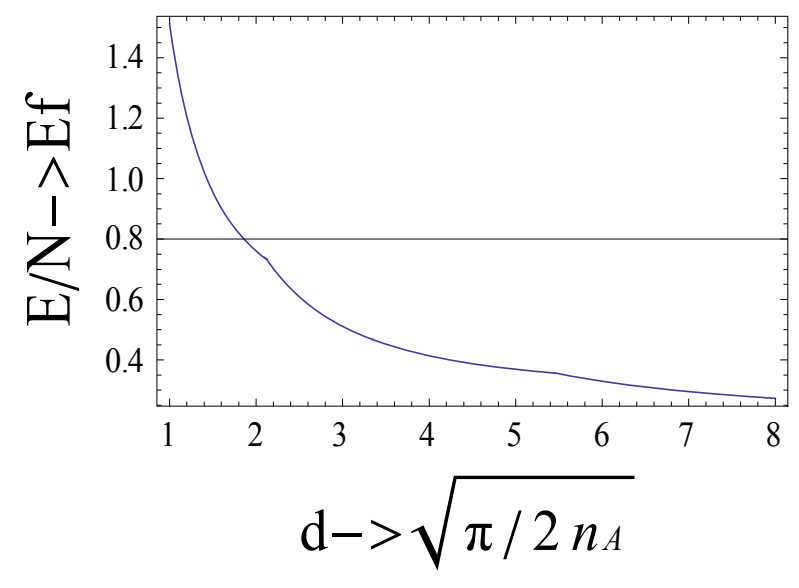

[A]

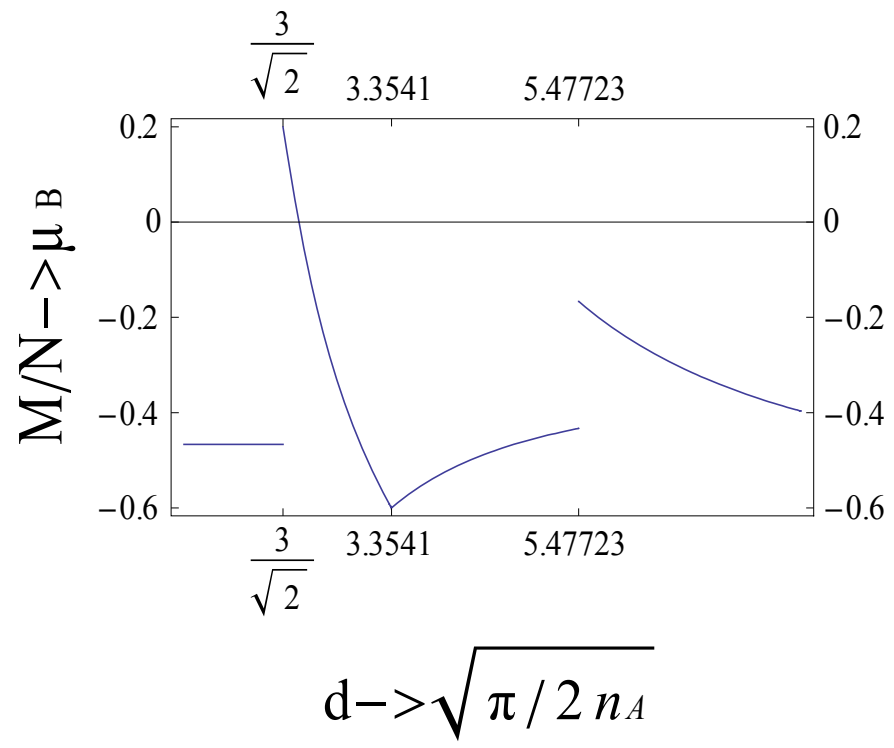




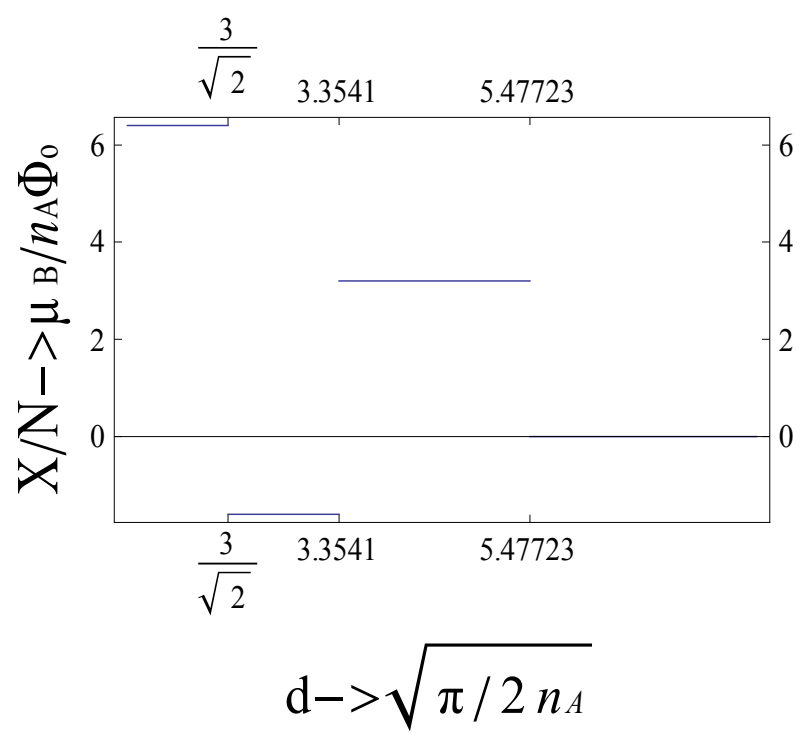

[C]

Fig 4.1.5: Graphs: [A] Energy, [B] Magnetisation and [C] Susceptibility as functions of thickness $d$ for $B=1 / 3 n_{A} \Phi$ o

These results appear to have a resemblance with the corresponding ones that we saw earlier in the main part of this section. The main difference is that the magnetisation discontinuities now occur more frequently.

We should note here that inclusion of such Zeeman splitting later in the full 3D problem will give rise in certain cases to pronounced local minima in energy (see end of Section 5).

Below, we also provide the corresponding 2D graph for the magnetisation:

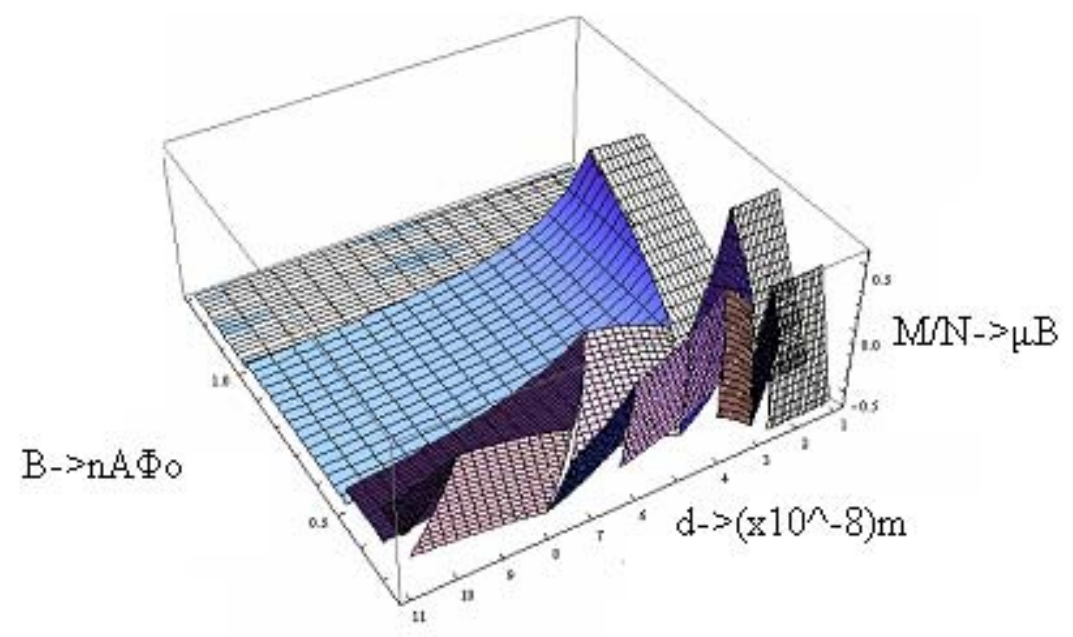

Fig 4.1.6: Magnetization as function of both $B$ and $d$.

[The corresponding "transport-related" discussion might have a relation to interesting "spin-Physics" at the edges because of the above Zeeman-induced spin-asymmetry. This is especially so if the system were folded into an Aharonov-Bohm cylinder (with nonzero thickness), because of Berry's phase effects on the opposite spins that effectively feel an inhomogeneous magnetic field due to the nonvanishing curvature. However, this interesting issue deserves a separate study.] 


\subsection{Inclusion of electron-electron interactions: Composite Fermions}

Although we found new transitions that correspond to partial LL filling and as already noted, we could be tempted to speculate that these might have something to do with fractional fillings and the FQHE if interactions were included, for consistency, we have chosen to restrict ourselves to noninteracting particles in the main part of this article. However, in this Section we will make an exception and consider briefly interacting electrons, because the same line of reasoning and the same general approach that was followed so far can also be followed for the so-called $\Lambda$-levels. These are the Landau Levels corresponding to a system of noninteracting Composite Fermions (CFs), the IQHE of CFs (i.e., with completely filled $\Lambda$-levels) corresponding, as is well known, to the FQHE of the original strongly interacting electron system.

Indeed, dealing with interactions between electrons inside a magnetic field is an extremely challenging problem in $2 \mathrm{D}$ [24] and it is even more so in the presence of a finite thickness, such as in the systems of our interest.

The picture of Composite Fermions (CFs) in 2D was devised and developed by Jain [25] and in this, each electron is, loosely speaking, attached to $2 p$ flux quanta $(p=$ integer) in order to create a CF (more rigorously there is a Chern-Simons transformation [24] that maps through a many-body Aharonov-Bohm transformation the strongly interacting system of electrons to almost noninteracting CFs). The CF method has been very successful in describing with very high accuracy, electron states in two dimensions that are in FQHE state. In our case, we have an extra $z$-direction and in principle, we are allowed to use Jain's method, because in our conventional system the planar and $z$-motions are decoupled and the (Chern-Simons) transformation performed to give the CFs only affects the 2D motion. Note, however, that this may not be a good model for topologically nontrivial systems.

Here, we remind the reader that in the approximation of noninteracting CFs, the energy spectrum of each $\mathrm{CF}$ is given by:

$$
\varepsilon_{n, k_{z}}=\hbar\left|\omega_{c}^{*}\right|(n+1 / 2)+\frac{\hbar^{2} k_{z}^{2}}{2 m^{*}}
$$

where $\omega_{c}^{*}=\frac{e B}{m^{*} c}$ (with $\mathrm{m}^{*}$ being the effective mass, which also depends on $B-$ see below) with $B^{*}=B-2 p \Phi_{o} n_{A}$ being the well-known effective magnetic field felt by the CFs ( $p$ being the integer mentioned earlier) and with the last term being the thickness-related contribution with again $k_{z}=\frac{\pi n_{z}}{d}, n_{z}=1,2,3 \ldots$. for rigid boundary conditions, as earlier.

Note that the same quantisation condition is valid for $k_{z}$, because it is not affected by the CF (or Chern-Simons) transformation. Let us choose as an example the integer $p$ to be unity, meaning that two flux quanta are attached to each electron. The Physics is now controlled by the effective magnetic field $B^{*}$, which determines the orbital $2 \mathrm{D}$ motion of CFs on the $x y$ plane. The degeneracy of $\Lambda$-levels now depends only on $B^{*}$ and the noninteracting CFs will have to be properly accommodated in the available $\Lambda$-levels.

Following the same method as earlier, we start from strong magnetic fields such as $\frac{1}{2} n_{A} \Phi_{o} \leq B^{*} \leq \infty$, so that only the lowest $\Lambda$-level is occupied. Simultaneously, by reversing the above with respect to $B$ and with $p=1$, the real magnetic field $B$ lies in the range:

$$
\frac{5}{2} n_{A} \Phi_{o} \leq B \leq \infty
$$

The total energy for this window is trivial:

$$
\begin{gathered}
E=N \varepsilon\left\{n=0, n_{z}=1\right\} \\
\Rightarrow \frac{E}{N}=\frac{\hbar e B^{*}}{2 m^{*} c}+\frac{\hbar^{2} \pi^{2}}{2 m^{*} d^{2}}
\end{gathered}
$$


with $N$ the number of CFs that is obviously the same as the number of electrons. We can choose to write the energy in units of 2D Fermi energy defined with the bare electronic mass $m$, in which case we have:

$$
\frac{E}{N}=E_{f}\left[\left(\frac{B^{*}}{n_{A} \Phi_{o}}\right) \frac{m}{m^{*}}+\left(\frac{\pi}{2 n_{A} d^{2}}\right) \frac{m}{m^{*}}\right],
$$

an expression valid, in the range (4.2.1), for every thickness $d$. The next $B^{*}$-window is naturally the following:

$$
\frac{1}{4} n_{A} \Phi_{o} \leq B^{*} \leq \frac{1}{2} n_{A} \Phi_{o}
$$

Here, we have two possible types of states to place the extra (namely N-2Ф*/Фo) CFs into: $\left\{n=0, n_{z}=2\right\}$ and $\left\{n=1, n_{z}=1\right\}$. The system will choose the minimum energy state in a way that depends strongly on the critical $d$-value determined by:

$$
\begin{gathered}
\varepsilon\left\{n=0, n_{z}=2\right\}=\varepsilon\left\{n=1, n_{z}=1\right\} \\
\frac{3 \hbar \omega_{c}^{*}}{2}+\frac{\hbar^{2} \pi^{2}}{2 m^{*} d^{2}}=\frac{\hbar \omega_{c}^{*}}{2}+\frac{4 \hbar^{2} \pi^{2}}{2 m^{*} d^{2}} \Rightarrow d_{\text {crit }}=\sqrt{\frac{3 \pi \Phi_{o}}{4 B^{*}}}
\end{gathered}
$$

which is equal to (4.4) with $B$ replaced by $B^{*}$. This actually occurs more generally in the energy results that follow and it is the universality mentioned earlier (a type of law of corresponding states). If thickness $d$ is smaller than (4.2.6), the extra CFs occupy $\left\{n=1, n_{z}=1\right\}$ states, while if $d$ is larger than (4.2.6), then $\left\{n=0, n_{z}=2\right\}$ states are preferred to be occupied. The total energy is then, for each case:

$$
\begin{aligned}
& E=2 \frac{\Phi^{*}}{\Phi_{o}} \varepsilon\left\{n=0, n_{z}=1\right\}+\left(N-2 \frac{\Phi^{*}}{\Phi_{o}}\right) \varepsilon\left\{n=1, n_{z}=1\right\} \\
& \Rightarrow \frac{E}{N}=E_{f}\left[-4\left(\frac{B^{*}}{n_{A} \Phi_{o}}\right)^{2} \frac{m}{m^{*}}+3\left(\frac{B^{*}}{n_{A} \Phi_{o}}\right) \frac{m}{m^{*}}+\left(\frac{\pi}{2 n_{A} d^{2}}\right) \frac{m}{m^{*}}\right], d \leq d_{\text {crit }} \\
& E=2 \frac{\Phi^{*}}{\Phi_{o}} \varepsilon\left\{n=0, n_{z}=1\right\}+\left(N-2 \frac{\Phi^{*}}{\Phi_{o}}\right) \varepsilon\left\{n=0, n_{z}=2\right\} \\
& \Rightarrow \frac{E}{N}=E_{f}\left[\left(\frac{B^{*}}{n_{A} \Phi_{o}}\right) \frac{m}{m^{*}}-6\left(\frac{B^{*}}{n_{A} \Phi_{o}}\right)\left(\frac{\pi}{2 n_{A} d^{2}}\right) \frac{m}{m^{*}}+\left(\frac{4 \pi}{2 n_{A} d^{2}}\right) \frac{m}{m^{*}}\right], d \geq d_{\text {crit }} .
\end{aligned}
$$

For the figures shown below, we use the following widely used approximation as an input:

$$
\frac{m}{m^{*}}=\frac{1.95}{\sqrt{\frac{B^{*}}{n_{A} \Phi_{o}}+2}}, \text { and we take } n_{A}=10^{16} \mathrm{el} / \mathrm{m}^{2}
$$




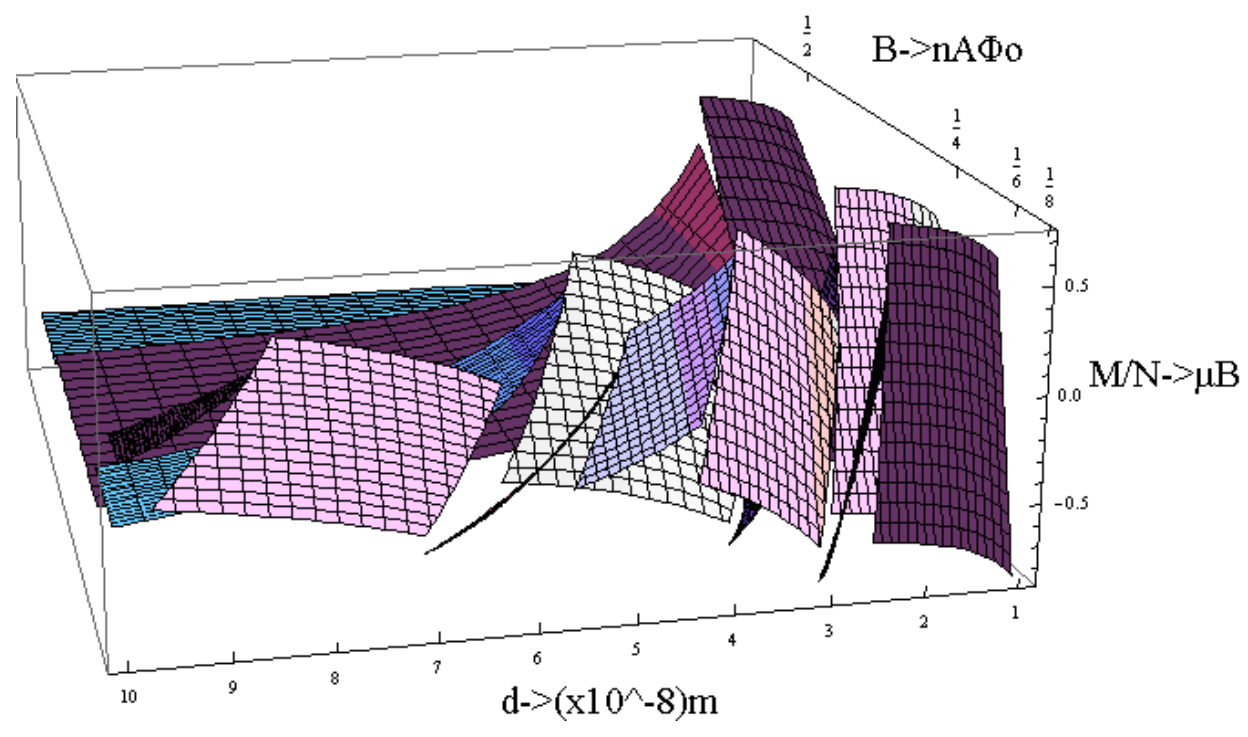

Fig. 4.2.1: Magnetisation as function of $B$ and $d$

Note that global magnetisation as a function of both $B$ and $d$ is considerably different from that given in Fig. 4.50. This is because interactions have a further significant role on thermodynamic properties through the $B$ dependent mass given above. Below, we also give a comparison between 1D graphs of magnetisation for CFs (left) and for noninteracting electrons (right), for $p=1$ and for corresponding states.

We can see some qualitative differences between the two systems, which deserve closer investigation, especially with respect to the noninteracting CFs approximation. Similarly, the issue of "internal transitions" (at partial $\Lambda$ level filling) for CFs is well beyond the scope of the present article.

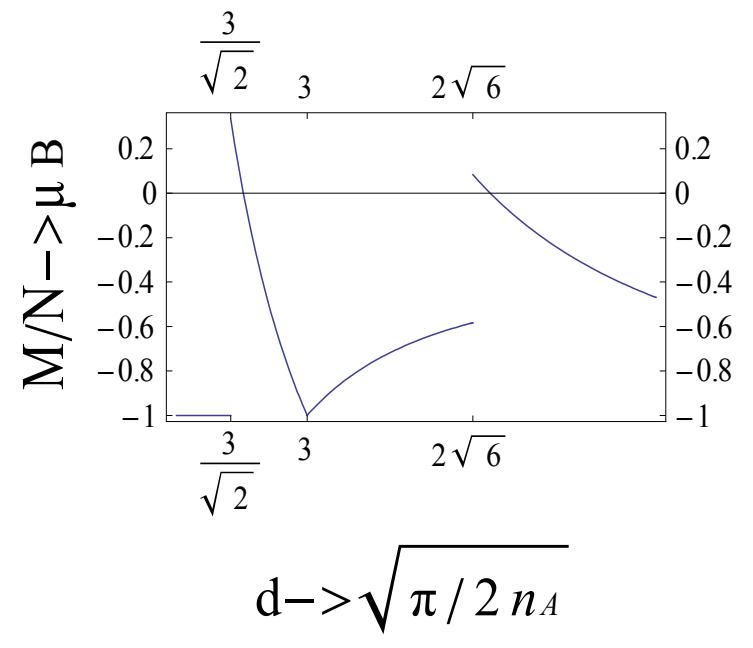

Interacting system

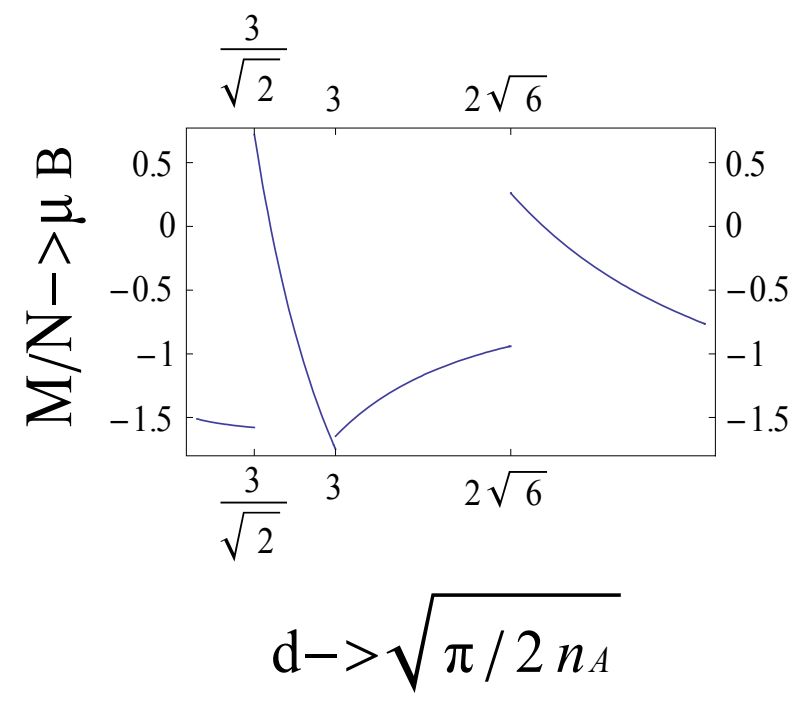

Noninteracting system

B

Figure 4.2.2: A): Magnetisation per Composite Fermion as a function of width $d$ for $B^{*}=1 / 6 n A \Phi o$ or $B=13 / 6 n_{A} \Phi o$. B): Magnetisation per electron as a function of width $d$ for $B=1 / 6 n A \Phi o$. 


\section{Electron gas inside a magnetic field in full 3D space}

For comparison, let us now deal with the case of noninteracting electrons in full 3D space with periodic boundary conditions imposed along the direction of the field. Although one might expect that things will now be getting more complicated, especially in the presence of a homogeneous magnetic field, this problem is actually more tractable than the earlier one of the finite-thickness interface and amenable to closed-form solutions for the thermodynamic functions. The basic origin of the simplification is the fact that $k_{z}$ is now a quasicontinuous variable. In fact, the quantum mechanical problem of 3D electron gas at zero temperature in a magnetic field was studied many years ago [27], in which the direct use of the grandcanonical potential $\Omega$ with generalised Riemann functions (or Hurwitz zeta functions) appeared in the results at low temperatures (see also [28]). Our aim in this section is to determine exactly the energetics of the ground state of noninteracting electrons by using a very different, simpler and more physical method of energy interplays, when the electron system occupies combined Landau Levels with (now quasicontinuous) $z$-axis levels, having always in mind the minimum total energy requirement at $\mathrm{T}=0$. Not surprisingly, it will transpire that all thermodynamic properties (e.g., Energy, Magnetisation and Susceptibility) will be determined analytically in terms of imaginary parts of Hurwitz zeta functions. However, it will also turn out from these exact solutions that we can determine the exact quantal manner in which the semi-classical dHvA periodicity is violated, which could be relevant for certain 3D solid state systems (but of very low density as we shall see). In this respect, our method is superior compared with earlier semi-classical approaches that do not address such violations.

We start by writing again the single-particle energies that emerge from the solution of the Schrodinger equation in space with cubic geometry (with length $L$ ), by now imposing periodic boundary conditions in the $z$-direction (the direction of the applied magnetic field). Then, the single-particle energy spectrum consists of the Landau Levels that describe the motion in the $x y$-plane, plus a (nonrelativistic) kinetic term (free wave) in the $z$-direction, namely:

$$
\varepsilon_{n}=\hbar \omega_{c}\left(n+\frac{1}{2}\right)+\frac{\hbar^{2} k_{z}{ }^{2}}{2 m}, \quad k_{z}=2 \pi \frac{n_{z}}{L}, \quad n_{z}=0, \pm 1, \pm 2 \ldots, \quad n \text { a nonnegative integer }
$$

with $L$ assumed macroscopic. We now have quasicontinuous $k_{z}$ (because $L \rightarrow \infty$ ) and strong quantisation in the $x y$-plane. Let us first study the Pauli principle-respecting occupational procedure. Electrons first occupy the lowest LL (for $n_{z}=0$ ) and then start building a 1D Fermi line segment along $k_{z}$ in $k$-space (with $k_{z}$ now taking also negative values). However, this cannot go on forever, even at $\mathrm{T}=0$. There comes a point when the length of the segment, essentially the Fermi wavenumber $k_{f}$ in $z$-direction, is so large that it is no longer energetically favourable to continue this procedure of occupations; it might be preferable for the extra electron to be excited to the next LL and start building a new Fermi segment from the beginning (notice, without violating the Pauli principle). Therefore, we can have a Fermi segment corresponding to any occupied LL but the number of such segments will depend on the values of $B$ and the electronic volume density $n_{V}$. As the above method is different from the usual semi-classical treatment, let us first work out the simplest examples.

Let us consider the case of extremely strong $B$ (in a range to be determined below), such that all electrons are frozen in the lowest LL $(n=0)$ and they form only a single Fermi segment (extending in $k$-space from $-k f_{1}$ to $+k f_{1}$ ). The maximum $k f_{1}$ will occur when, energetically speaking (in the spirit of the above) another $k_{f 2}$, associated with the $n=1 \mathrm{LL}$, is just about to form and this will occur when

$$
\hbar \omega_{c}=\frac{\hbar^{2} k_{f 1}^{2}}{2 m}
$$

and then with the standard substitution of a sum over the quasicontinuous $k_{z}$ with an integral in the limit of infinite $L$, we can determine $k f_{1}$ as follows:

$$
N=\frac{2 \Phi}{\Phi_{o}} \sum_{\vec{k}: \text { occupied }} 1 \rightarrow \frac{2 \Phi}{\Phi_{o}} \frac{L}{2 \pi} \int_{-k_{1}}^{k_{1}} d k
$$

from which it turns out that $k f_{1}=\pi^{2} l_{B}^{2} n_{V}$, where $l_{B}=\sqrt{\hbar c / e B}$ is the magnetic length and $n_{V}=N / V$ is the volume density, always for spinfull electrons; note that in astrophysical applications, there is usually an extra factor of 2 involved [26]. 
Then, by using (5.2), (5.3) and $\omega_{c}=\frac{e B}{m c}$, we obtain:

$$
n_{\text {crit } 1}=\left(\frac{16}{\pi}\right)^{1 / 2}\left(\frac{B}{\Phi_{o}}\right)^{3 / 2},
$$

which for fixed $B$ gives the critical density below which we have the above assumed case of only a single LL participating in the occupational process. More interesting, however, is the case of fixed $n_{V}$. Then (5.4) gives the critical magnetic field:

$$
B_{\text {crit } 1}=\left(\frac{\pi}{16}\right)^{1 / 3} n_{V}{ }^{2 / 3} \Phi_{o}
$$

in the sense that, it is only for $B>B_{\text {crit } 1}$ that we have the above scenario (of only a single LL being involved). In that case, the total energy is:

$$
E=\underbrace{\left[\left(\frac{2 \Phi}{\Phi_{o}}\right) \times \frac{L_{z}}{2 \pi} \int_{-k_{f}}^{k_{f}} d k_{z}\right]}_{N} \times \frac{\hbar \omega_{c}}{2}+\left(\frac{2 \Phi}{\Phi_{o}}\right) \times \frac{L_{z}}{2 \pi} \frac{\hbar^{2}}{2 m} \int_{-k_{f}}^{k_{f}} k_{z}^{2} d k_{z}
$$

$\Rightarrow E=N \frac{\hbar \omega_{c}}{2}+\frac{1}{3} N \frac{\hbar^{2} k_{f}^{2}}{2 m}$ or in units of 3D Fermi energy $\left(E_{f}=\frac{\hbar^{2} \kappa_{f}^{2}}{2 m}\right.$ and $\left.\kappa_{f}=\left(3 \pi^{2} n_{V}\right)^{2 / 3}\right)$ :

$$
\frac{E}{N}=\frac{E_{f}}{\left(3 \pi^{2}\right)^{2 / 3}}\left[2 \pi\left(\frac{B}{n_{V}^{2 / 3} \Phi_{o}}\right)+\frac{\pi^{2}}{12}\left(\frac{\Phi_{o} n_{V}^{2 / 3}}{B}\right)^{2}\right] .
$$

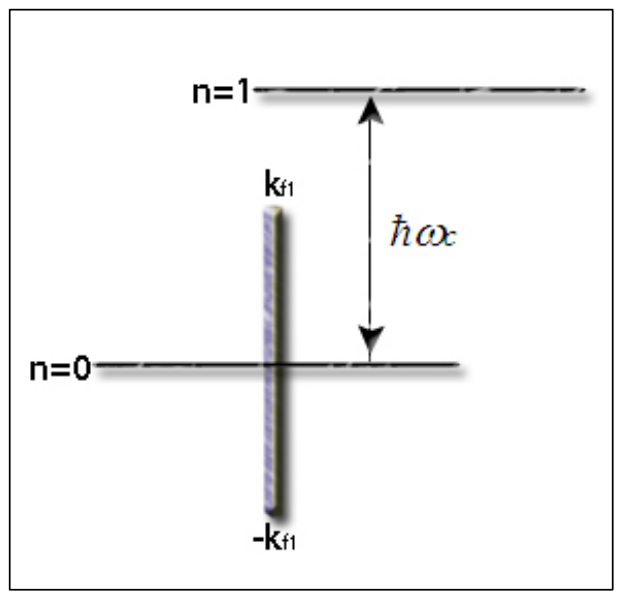

Fig. 5.1: Only one Fermi segment is created when $B>B_{\text {crit } 1}$

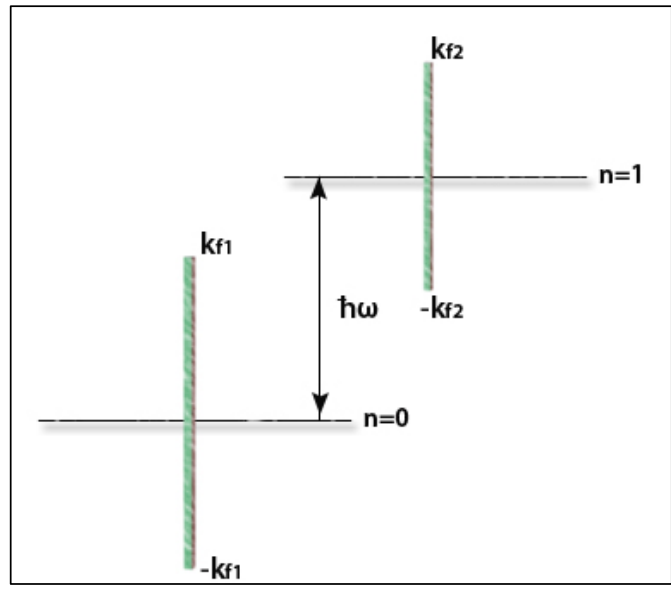

Fig. 5.2: Two Fermi segments are created when $B<B_{\text {crit } 1}$ (and also when $B>B_{\text {crit } 2}($ see (5.10))

If we now drop $B$ to a value slightly lower than $B_{\text {crit } 1}$, the lowest LL cannot accommodate all the electrons and the next LL (for $n=1$ ) will have to be used. We then start having a second Fermi line segment forming (extending from $-k_{f 2}$ to $+k_{f 2}$ ) associated with the $n=1 \mathrm{LL}$, while we simultaneously also have a first Fermi segment (with a $k_{f 1}$, always associated with the $n=0 \mathrm{LL}$ ) that now increases in size as we keep placing more electrons. The actual manner in which we now place the remaining electrons in the two LLs is back and forth in both of them, in a way that the "Fermi height" of the segment associated with $n=0$ and the one associated with $n$ $=1$ will both keep increasing and will at every point (for every density) be related with each other through the "equilibrium relation": 


$$
\frac{\hbar \omega_{c}}{2}+\frac{\hbar^{2} k_{f 1}^{2}}{2 m}=\frac{3 \hbar \omega_{c}}{2}+\frac{\hbar^{2} k_{f 2}^{2}}{2 m}
$$

where $k f_{1}=\pi^{2} l_{B}^{2} n_{1}$ and $k f_{2}=\pi^{2} l_{B}^{2} n_{2}$ (coming out from an argument exactly like the one in (5.3) but now with partial densities). For any given volume density $n_{V},(5.7)$ will determine the proper (energetically favourable) partition $\left(n_{1}, n_{2}\right)$ of the total density between the two LLs involved. Again, (5.7) reflects the fact that the extra electron at every point of the occupational procedure must have the same single-particle energy for either of the two options (or scenarios). If (5.7) were not satisfied and one side were larger than the other was, it would indicate that the occupational procedure followed up to that point energetically was not the lowest. Compare the above "equilibrium condition" with the one that was implemented in (3.5) of Section 3, or (4.3) of Section 4, when only two QW levels were occupied. Note that, although the cases are different, they are along a similar line of reasoning.

From (5.7) and the expressions for $k f_{1}$ and $k f_{2}$, we obtain the optimal density partition in the two LLs (by also utilising $\left.n_{V}=n_{1}+n_{2}\right)$, the final result being:

$$
\left.\begin{array}{l}
n_{1}=\frac{n_{V}}{2}\left(1+\frac{B^{3}}{B_{c r i t 1}^{3}}\right) \\
n_{2}=\frac{n_{V}}{2}\left(1-\frac{B^{3}}{B_{c r i t 1}^{3}}\right)
\end{array}\right\},
$$

where $B_{c r i t 1}$ is given by (5.5). The above partition of density is valid only for $B<B_{\text {crit } 1}$. With regard to the lowest value of $B$ allowed, i.e., the complete range of $B$-values where (5.8) is valid, see further below. Note how the full three-dimensionality and the extra presence of the magnetic field have modified the earlier found partition (3.9).

The total energy in the above case will be determined by:

$$
\begin{gathered}
E=N_{1} \frac{\hbar \omega_{c}}{2}+\frac{1}{3} N_{1} \frac{\hbar^{2} k_{f 1}^{2}}{2 m}+N_{2} \frac{3 \hbar \omega_{c}}{2}+\frac{1}{3} N_{2} \frac{\hbar^{2} k_{f 2}^{2}}{2 m} \\
\Rightarrow \frac{E}{N}=\frac{E_{f}}{\left(3 \pi^{2}\right)^{2 / 3}}\left[4 \pi\left(\frac{B}{n_{V}^{2 / 3} \Phi_{o}}\right)+\frac{\pi^{2}}{48}\left(\frac{\Phi_{o} n_{V}^{2 / 3}}{B}\right)^{2}-16\left(\frac{B}{n_{V}^{2 / 3} \Phi_{o}}\right)^{4}\right] .
\end{gathered}
$$

The lower value of the range of $B$ can then be determined by considering the next case, namely, when a $3^{\text {rd }}$ Fermi segment (of LL index $n=2$ ) is about to form, for which we have the equilibrium condition $\frac{\hbar \omega_{c}}{2}+\frac{\hbar^{2} k_{f 1}^{2}}{2 m}=\frac{5 \hbar \omega_{c}}{2}$ or equivalently, $\frac{3 \hbar \omega_{\mathrm{c}}}{2}+\frac{\hbar^{2} \mathrm{k}_{\mathrm{f} 2}^{2}}{2 \mathrm{~m}}=\frac{5 \hbar \omega_{\mathrm{c}}}{2}$ and turns out to be

$$
B_{\text {crit } 2}=(3-2 \sqrt{2})^{1 / 3} B_{\text {crit } 1}
$$

Following these two examples, to proceed further with the most general case requires greater mathematical sophistication. In the most general case, in every $i^{\text {th }}$ LL, electrons build a 1D Fermi segment that defines a Fermi wavevector $k_{f_{i}}$, where the index $i$ (defined by $i=n+1, n$ is a LL index) runs over all occupied LLs and has positive integer values. When the magnetic field is a constant $B$, let us say that we know that the system occupies in general $k$ LLs $(k \geq 1)$ and creates $k$ 1D Fermi segments in the $z$-axis (and then $i$ runs from 1 to $k$ ). The associated $k_{f_{i}}$ s must be determined as in the example shown above, namely: 


$$
N_{i}=\frac{2 \Phi}{\Phi_{o}} \sum_{\vec{k}: \text { occupied }} 1 \rightarrow \frac{2 \Phi}{\Phi_{o}} \frac{L}{2 \pi} \int_{-k_{i}}^{k_{i}} d k \Rightarrow k f_{i}=\pi^{2} l_{B}^{2} n_{i}
$$

where $n_{i}=N_{i} / V$ is the partial volume density corresponding to the $i^{\text {th }}$ LL $(i=1,2,3 \ldots k)$. A similar line of reasoning as that of Section 2 must then be followed. The last electrons on the ends of any of the $k$ 1D Fermi segments must have equal single-particle energies, i.e., in the spirit of Section 2, 'equilibrium' is satisfied; otherwise, we would have transitions and rearrangements between the states, such that equilibrium is recovered, to assure that the energetically optimal occupational procedure has been followed.

The appropriate mathematical expression for the equilibrium is then:

$$
\frac{\hbar^{2} k_{f 1}^{2}}{2 m}+\frac{\hbar \omega_{c}}{2}=\frac{\hbar^{2} k_{f 2}^{2}}{2 m}+\frac{3 \hbar \omega_{c}}{2}=\ldots=\frac{\hbar^{2} k_{f k}^{2}}{2 m}+\hbar \omega_{c}\left(k-\frac{1}{2}\right) .
$$

From the above condition and with the use of (5.11), we can determine in the general case (i.e., for any $k$ ) the proper partition of all $1 \mathrm{D}$ densities in each LL:

$$
\begin{gathered}
n_{i}^{2}=n_{1}^{2}-(i-1) \frac{B^{3}}{\left(\frac{\pi}{16}\right) \Phi_{0}^{3}}, \\
n_{V}=\sum_{i=1}^{k} n_{i},
\end{gathered}
$$

where index $i$ runs from 1 to $k$ and $n_{V}$ denotes the total (global) volume density of electrons. This is a system of $k$ equations with $k$ unknown variables, which can be solved analytically. We will return to this solution soon. Let us first think of the appropriate values of magnetic field $B$ that force the system to occupy exactly $k$ LLs; from the equilibrium condition (5.12) we can find a critical value of $B$ as a function of $n_{1}$. When $B$ is exactly equal to this critical value, electrons start the occupation of $k+1^{\text {th }}$ LL. However, this is rather easy to describe; it occurs when the 1D Fermi segment at the $k+1^{\text {th }}$ LL is just about to be formed, namely:

$$
\frac{\hbar^{2} k_{f 1}^{2}}{2 m}=k \hbar \omega_{c}
$$

such that $B$ is just

$$
B_{c r i t}^{3}(k)=\frac{1}{k}\left(\frac{\pi}{16}\right) \Phi_{0}^{3} n_{1}^{2},
$$

by following steps similar to the ones followed to derive (5.5) - but note that now $n_{1}$ also depends on $B$.

The same line of reasoning gives the other critical value of $B$, which makes electrons start the occupation of the $k^{\text {th }}$ LL:

$$
B_{\text {crit }}^{3}(k-1)=\frac{1}{k-1}\left(\frac{\pi}{16}\right) \Phi_{0}^{3} n_{1}^{2}
$$

and all the previous conditions are correct only in the case that the magnetic field varies in the following window:

$$
B_{\text {crit }}(k-1) \geq B \geq B_{\text {crit }}(k),
$$

this being true for $k>1$; for $k=1$, we only have $B \geq B_{\text {crit }}(1)$.

Here, we remind the reader that the first linear density $n_{1}$ also depends on the magnetic field and it must be calculated analytically. Now, writing (5.13) in a more convenient form, we obtain: 


$$
n_{i}=n_{V} \frac{\sqrt{\frac{B_{\text {crit } 1}^{3}}{B^{3}}\left(\frac{n_{1}}{n_{V}}\right)^{2}-(i-1)}}{\sqrt{\frac{B_{c r i t 1}^{3}}{B^{3}}}},
$$

where we set $B_{\text {crit } 1}^{3}=\left(\frac{\pi}{16}\right) \Phi_{0}^{3} n_{V}{ }^{2}$ that was found to be the first critical value of $B$ (see (5.5)). It is also convenient to define a quantity (a filling factor-like quantity):

$$
a_{1}=\frac{B_{c r i t 1}^{3}}{B^{3}}\left(\frac{n_{1}}{n_{V}}\right)^{2} .
$$

It is then easy to observe that when $B=B_{\text {crit }}(k)$ (see (5.16)), $a_{1}=k$ and when $B=B_{\text {crit }}(k-1)$ (see (5.17)), $a_{1}=k-1$, so it must hold that:

$$
B_{\text {crit }}(k-1) \geq B \geq B_{\text {crit }}(k) \Rightarrow k-1 \leq a_{1} \leq k \text {. }
$$

When $B$ lies on a critical value, then $a_{1}$ is an integer ( $k$ or $k-1$ accordingly), otherwise it must be a fractional (more generally irrational) real number. Then (5.19) becomes:

$$
n_{i}=n_{V} \frac{\sqrt{a_{1}-i+1}}{\sqrt{\frac{B_{c r i t 1}^{3}}{B^{3}}}},
$$

i.e., the coefficient of $n_{V}$ is just the percentage of density that corresponds to the $(i)_{\text {th }}$ LL. Now, by using $(5.14)$ we have:

$$
\sqrt{\frac{B_{c r i t 1}^{3}}{B^{3}}}=\sum_{i=1}^{k} \sqrt{a_{1}-i+1}
$$

or

$$
\sqrt{\frac{B_{c r i t 1}^{3}}{B^{3}}}=\sqrt{a_{1}}+\sqrt{a_{1}-1}+\sqrt{a_{1}-2}+\ldots+\sqrt{a_{1}-(k-1)} .
$$

Unfortunately, it does not seem possible to solve the above equation with respect to $a_{1}$. However, one observes that (5.23) can be written with the use of generalised Riemann or Hurwitz zeta functions (defined by $\left.\zeta(s, a)=\sum_{i=0}^{\infty} 1 /(i+a)^{s}\right)$, as follows:

$$
\sqrt{\frac{B_{c r i t 1}^{3}}{B^{3}}}=-i\left[\zeta\left(-1 / 2,-a_{1}\right)-\zeta\left(-1 / 2, k-a_{1}\right)\right]
$$

where $i$ is the imaginary unit and $k$ the number of occupied LLs and $k-1 \leq a_{1} \leq k, 0 \leq k-a_{1} \leq 1$. Therefore, the difference between Hurwitz zeta functions must be a pure complex number:

$$
\operatorname{Re}\left\{\zeta\left(-1 / 2,-a_{1}\right)\right\}=\operatorname{Re}\left\{\zeta\left(-1 / 2, k-a_{1}\right)\right\} \forall a_{1}
$$

and it is also true that $\operatorname{Im}\left\{\zeta\left(-1 / 2, k-a_{1}\right)\right\}=0$, because $k-a_{1} \geq 0$. 
Finally, we find that

$$
\begin{gathered}
\zeta\left(-1 / 2,-a_{1}\right)-\zeta\left(-1 / 2, k-a_{1}\right)=i \operatorname{Im}\left\{\zeta\left(-1 / 2,-a_{1}\right)\right\} \\
\operatorname{Im}\left\{\zeta\left(-1 / 2,-a_{1}\right)\right\}=\sqrt{\frac{B_{\text {crit } 1}^{3}}{B^{3}}} .
\end{gathered}
$$

This is the key to the solution of this problem; only the imaginary part of the Hurwitz zeta functions has physical meaning. With the help of (5.28), (5.16) and (5.17), we can then write down analytical expressions for the critical values of $B$ that do not depend on $n_{1}$ :

$$
\begin{gathered}
B_{\text {crit }}(k)=\frac{B_{\text {crit } 1}}{(\operatorname{Im}\{\zeta(-1 / 2,-k)\})^{2 / 3}} \\
B_{\text {crit }}(k-1)=\frac{B_{\text {crit } 1}}{(\operatorname{Im}\{\zeta(-1 / 2,-(k-1))\})^{2 / 3}} .
\end{gathered}
$$

As a test of consistency, we can check that the above reproduce the earlier results of (5.5) and (5.10) (see (5.34) below for the imaginary part of the Hurwitz Zeta functions). For $k=1$, then:

$$
\begin{gathered}
B_{\text {crit }}(1)=\frac{B_{\text {crit } 1}}{(\operatorname{Im}\{\zeta(-1 / 2,-1)\})^{2 / 3}}=\frac{B_{\text {crit } 1}}{1}=B_{\text {crit } 1}, \text { which is }(5.5) \text { and for } k=2 \text { we have } \\
B_{\text {crit }}(2)=\frac{B_{\text {crit } 1}}{(\operatorname{Im}\{\zeta(-1 / 2,-2)\})^{2 / 3}}=\frac{1}{(1+\sqrt{2})^{2 / 3}} B_{\text {crit } 1}=(3-2 \sqrt{2})^{1 / 3} B_{\text {crit } 1} \text {, which is }(5.10) .
\end{gathered}
$$

It is also interesting to check what the differences of neighbouring inverse $B_{c r i t} \mathrm{~s}$ are and relate their behavioural pattern to the standard period of the de Haas-van Alphen effect. It is true that in weak magnetic fields and hence, large values of $k$, the system starts behaving semi-classically (then the segment sizes will come from cuts of Landau tubes inside a Fermi sphere) and then we expect an oscillating period similar to that of the dHvA effect. Having calculated the critical values of $B$ analytically, we have the ability to check the period directly, without any approximations. Indeed, the semi-classical $\mathrm{dHvA}$ period is:

$$
\delta\left(\frac{1}{B}\right)=\frac{4 \pi}{\left(3 \pi^{2}\right)^{2 / 3}} \frac{1}{n^{2 / 3} \Phi_{o}}=\frac{\left(\frac{4}{9}\right)^{1 / 3}}{B_{\text {crit } 1}}=\frac{0.76314}{B_{\text {crit } 1}} .
$$

The difference of inverse $B$ that we have found is (from (5.29) and (5.30)):

$$
\delta\left(\frac{1}{B}\right)=\frac{1}{B_{\text {crit }}(k)}-\frac{1}{B_{\text {crit }}(k-1)}=\frac{(\operatorname{Im}\{\zeta(-1 / 2,-k)\})^{2 / 3}-(\operatorname{Im}\{\zeta(-1 / 2,-(k-1))\})^{2 / 3}}{B_{\text {crit } 1}} .
$$

Note that when $k=1$, then $\delta(1 / B)=1 / B_{\text {crit } 1}$, which deviates from (5.31) by about $31 \%$, while if $k=2$, then $\delta(1 / B)=0.7996 / B_{c r i t 1}$, which deviates from (5.31) by only $5 \%$. Now, comparing (5.31) with (5.32), leads to the conclusion that the following must be proven (for large $k$ ):

$$
(\operatorname{Im}\{\zeta(-1 / 2,-k)\})^{2 / 3}-(\operatorname{Im}\{\zeta(-1 / 2,-(k-1))\})^{2 / 3} \approx\left(\frac{4}{9}\right)^{1 / 3}
$$


Using the well-known relations (which are true, because $k$ is an integer):

$$
\operatorname{Im}\{\zeta(-1 / 2,-k)\}=\sum_{j=1}^{k} \sqrt{j} \text { and } \operatorname{Im}\{\zeta(-1 / 2,-(k-1))\}=\sum_{j=1}^{k-1} \sqrt{j}
$$

then the following must be proven

$$
\left(\sum_{j=1}^{k} \sqrt{j}\right)^{2 / 3}-\left(\sum_{j=1}^{k-1} \sqrt{j}\right)^{2 / 3} \approx\left(\frac{4}{9}\right)^{1 / 3}=\left(\frac{2}{3}\right)^{2 / 3} \text { when } k>>1
$$

For this, let us think momentarily in a slightly different manner. Instead of calculating $\delta\left(\frac{1}{B}\right)$, we can calculate $\delta\left(\frac{1}{B}\right)^{3 / 2}$ and then relate it to $\delta\left(\frac{1}{B}\right)$. Using (5.34) and (5.32) we obtain:

$$
\delta\left(\frac{1}{B}\right)^{3 / 2}=\frac{\sqrt{k}}{B_{c r i t 1}^{3 / 2}}
$$

Equivalently, we can write $\delta\left(\frac{1}{B}\right)$ as:

$$
\delta\left(\frac{1}{B}\right)=\frac{2}{3} \delta\left(\frac{1}{B}\right)^{3 / 2} \sqrt{B_{c r i t}(k)}=\frac{2}{3} \frac{1}{B_{\text {crit } 1}} \frac{\sqrt{k}}{(\operatorname{Im}\{\zeta(-1 / 2,-k)\})^{1 / 3}}
$$

and now comes the approximation. For large $k$ (weak magnetic fields), we must expand the term $\frac{\operatorname{Im}\{\zeta(-1 / 2,-k)\}}{(\sqrt{k})^{3}}$ around $k=\infty$, to see that it is almost equal to $2 / 3$, which is indeed true:

$$
\frac{\operatorname{Im}\{\zeta(-1 / 2,-k)\}}{(\sqrt{k})^{3}}=\frac{2}{3}+\frac{1}{2 k}+\left(\frac{1}{k}\right)^{3 / 2} \zeta\left(-\frac{1}{2}\right)+\frac{1}{24 k^{2}}-\frac{1}{1920 k^{4}}+\mathrm{O}\left\{\left(\frac{1}{k}\right)^{11 / 2}\right\} \approx \frac{2}{3}
$$

Therefore, the result is:

$$
\delta\left(\frac{1}{B}\right)=\frac{2}{3}\left(\frac{3}{2}\right)^{1 / 3} \frac{1}{B_{c r i t 1}}=\left(\frac{2}{3}\right)^{2 / 3} \frac{1}{B_{c r i t 1}}
$$

as anticipated (see (5.31)). The conclusion is that in magnetic fields that are not extremely strong (i.e. for many LLs occupied), the system rapidly converges to the semi-classical behaviour. However, this semi-classical dHvA period is violated at exceedingly strong magnetic fields.

Unfortunately, the magnetic fields necessary in order to observe these extreme quantum effects are very large and therefore, we cannot see them in the laboratory. However, we can effectively reduce them by lowering the value of electronic number density. For example, consider (5.5), which gives the first critical value of $B$ (the largest of all critical values). Nowadays, we might achieve magnetic fields up to 40 Tesla, so:

$$
n_{\max }=\left(\frac{16}{\pi}\right)^{1 / 2}\left(\frac{40 T}{\Phi_{o}}\right)^{3 / 2}=2.17 \times 10^{24} m^{-3}
$$


This can be considered as the maximum number density of charge carriers that a material must have in order for our extreme quantum results (reflected in the dHvA violations) to be seen experimentally. The above density is four orders of magnitude smaller than typical metallic densities.

The final important step for this section is to calculate the total energy and magnetisation. The energy is just a sum over all occupied LLs and $z$-axis levels:

$$
E=\sum_{j=0}^{k-1}\left(N_{j+1} \hbar \omega(j+1 / 2)+\frac{1}{3} N_{j+1} \frac{\hbar^{2} k f_{j+1}^{2}}{2 m}\right)
$$

where $k f_{j+1}^{2}=\pi^{4} l_{B}^{4} n_{j+1}^{2}$ (from (5.11)), which leads to:

$$
E=\sum_{j=0}^{k-1}\left(\hbar \omega j N_{j+1}+\frac{\hbar \omega N_{j+1}}{2}+\frac{1}{3} \frac{\hbar^{2} \pi^{4} l_{B}^{4}}{2 m} N_{j+1} n_{j+1}^{2}\right)
$$

Using $N_{j+1}=n_{j+1} V$ and substituting $n_{j}$ with its equal from (5.13), we find:

$$
E=\sum_{j=0}^{k-1}\left(\hbar \omega N_{j+1} \frac{B_{c r i t 1}^{3}}{B^{3}} \frac{n_{1}^{2}}{n^{2}}+\frac{\hbar \omega N_{j+1}}{2}+\frac{1}{3} \frac{\hbar^{2} \pi^{4} l_{B}^{4}}{2 m} V n_{j+1}^{3}-\hbar \omega V \frac{B_{c r i t 1}^{3}}{B^{3}} \frac{n_{j+1}^{3}}{n^{2}}\right)
$$

Now, observing that

$$
\frac{\hbar^{2} \pi^{4} l_{B}^{4}}{2 m}=\hbar \omega \frac{B_{c r i t 1}^{3}}{B^{3} n^{2}}
$$

the energy becomes

$$
E=\sum_{j=0}^{k-1}\left(\hbar \omega N_{j+1} \frac{B_{c r i t 1}^{3}}{B^{3}} \frac{n_{1}^{2}}{n^{2}}+\frac{\hbar \omega N_{j+1}}{2}-\frac{2}{3} \hbar \omega V \frac{B_{c r i t 1}^{3}}{B^{3}} \frac{n_{j+1}^{3}}{n^{2}}\right)
$$

The first term gives

$$
\sum_{j=0}^{k-1} \hbar \omega N_{j+1} \frac{B_{c r i t 1}^{3}}{B^{3}} \frac{n_{1}^{2}}{n^{2}}=N \hbar \omega \frac{B_{c r i t 1}^{3}}{B^{3}} \frac{n_{1}^{2}}{n^{2}}=N \hbar \omega a_{1}
$$

the second term gives

$$
\sum_{j=0}^{k-1} N_{j+1} \frac{\hbar \omega}{2}=N \frac{\hbar \omega}{2}
$$

and the third term gives

$$
\frac{2}{3} \hbar \omega V \frac{B_{c r i t 1}^{3}}{B^{3}} \sum_{j=0}^{k-1} \frac{n_{j+1}^{3}}{n^{2}}=\frac{2}{3} \hbar \omega N\left(\frac{B^{3}}{B_{c r i t 1}^{3}}\right)^{1 / 2} \sum_{j=0}^{k-1}\left(a_{1}-j\right)^{3 / 2}
$$

Now, the sum $\sum_{j=0}^{k-1}\left(a_{1}-j\right)^{3 / 2}$ is just a difference of two zeta functions of order $-3 / 2$ : 


$$
\sum_{j=0}^{k-1}\left(a_{1}-j\right)^{3 / 2}=i\left[\zeta\left(-3 / 2,-a_{1}\right)-\zeta\left(-3 / 2, k-a_{1}\right)\right] .
$$

Energy must be a real quantity; therefore, it must hold that:

and

$$
\begin{gathered}
\zeta\left(-3 / 2,-a_{1}\right)-\zeta\left(-3 / 2, k-a_{1}\right)=i \operatorname{Im}\left\{\zeta\left(-3 / 2,-a_{1}\right)\right\} \\
\sum_{j=0}^{k-1}\left(a_{1}-j\right)^{3 / 2}=-\operatorname{Im}\left\{\zeta\left(-3 / 2,-a_{1}\right)\right\} .
\end{gathered}
$$

Finally, the energy per electron is:

$$
E / N=\hbar \omega\left[1 / 2+a_{1}+\frac{2}{3}\left(\frac{B^{3}}{B_{\text {crit } 1}^{3}}\right)^{1 / 2} \operatorname{Im}\left\{\zeta\left(-3 / 2,-a_{1}\right)\right\}\right] \text {. }
$$

Once again, it does not seem possible to solve (5.28) with respect to $a_{1}$ (there is no analytic expression for the inverse function of the imaginary part of the Hurwitz zeta functions). However, this is not quite necessary, because we can solve (5.28) numerically and then determine the values of (5.52) for every $B$. If up to this point all our calculations are correct, the derivative of energy with respect to $a_{1}$ must vanish, i.e., energy is indeed minimal and the correct density distributions are given by (5.52). Although tedious, it is straightforward to check this expectation and indeed, we have:

$$
\frac{\partial(E / N)}{\partial a_{1}}=\hbar \omega_{c}\left[1-\frac{2}{3}\left(\frac{B^{3}}{B_{c r i t 1}^{3}}\right)^{1 / 2} \frac{\partial}{\partial a_{1}}\left[\sum_{j=0}^{k-1}\left(a_{1}-j\right)^{3 / 2}\right]=\hbar \omega_{c}\left[1-\left(\frac{B^{3}}{B_{c r i t 1}^{3}}\right)^{1 / 2} \sum_{j=0}^{k-1}\left(a_{1}-j\right)^{1 / 2}\right]=\hbar \omega_{c}\left[1-\left(\frac{B^{3}}{B_{c r i t 1}^{3}}\right)^{1 / 2}\left(\frac{B_{c r i t 1}^{3}}{B^{3}}\right)^{1 / 2}\right]=0\right.
$$

At the critical values of $B$ (the ones expressed by (5.29)), the energy has a simple analytic form, namely:

$$
E / N=\frac{\hbar e B_{c r i t 1}}{m c}\left[\frac{1}{(\operatorname{Im}\{\zeta(-1 / 2,-k)\})^{2 / 3}}\right]\left[1 / 2+k+\frac{2}{3} \frac{\operatorname{Im}\{\zeta(-3 / 2,-k)\}}{\operatorname{Im}\{\zeta(-1 / 2,-k)\}}\right]
$$

where $k$ arises from the inversion of (5.29), actually labelling the critical point. Note the amusing fact that

$$
\hbar e B_{c r i t 1} / m c=(2 / 3)^{2 / 3} \frac{\hbar^{2}\left(3 \pi^{2} n_{V}\right)^{2 / 3}}{2 m}=(2 / 3)^{2 / 3} E_{f}(3 D),
$$

where $E_{f}(3 D)=\frac{\hbar^{2}\left(3 \pi^{2} n_{V}\right)^{2 / 3}}{2 m}$, the 3D Fermi energy of electrons when no magnetic field is applied on the cube (something that could be seen directly from (5.5) as well).

In the limit $B \rightarrow 0$ (or $k \rightarrow \infty),(5.53)$ can be shown to tend to $(3 / 5) E_{f}(3 D)$. This can also be seen from Fig. 5.3 below.

Finally, by taking the derivative of (5.52), we can also determine analytically the magnetisation per electron using the relation:

$$
M=-\frac{\partial E}{\partial B}
$$

However, we will need to know the derivative of $a_{1}$ with respect to $B$; this can be calculated from (5.19) and the result is 


$$
\begin{aligned}
& \frac{d a_{1}}{d B}=\frac{-3 B^{-5 / 2} B_{c r i t}^{3 / 2}}{i\left[\zeta\left(1 / 2,-a_{1}\right)-\zeta\left(1 / 2, k-a_{1}\right)\right]}=\frac{3 B^{-5 / 2} B_{c r i t}^{3 / 2}}{\operatorname{Im}\left\{\zeta\left(1 / 2,-a_{1}\right)\right\}} \\
& \Rightarrow M / N=-\mu_{\mathrm{B}}\left[1+2 a_{1}+\frac{10}{3} x^{3 / 2} \operatorname{Im}\left\{\zeta\left(-3 / 2,-a_{1}\right)\right\}\right]
\end{aligned}
$$

and for the magnetic susceptibility, the corresponding procedure gives:

$$
X / N=\frac{\mu_{\mathrm{B}}}{B_{\text {crit } 1}}\left[9 x^{-5 / 2} \frac{1}{\operatorname{Im}\left\{\zeta\left(1 / 2,-a_{1}\right)\right\}}-5 x^{1 / 2} \operatorname{Im}\left\{\zeta\left(-3 / 2,-a_{1}\right)\right\}\right],
$$

where $\mu_{\mathrm{B}}=\frac{\hbar e}{2 m c}$ is the Bohr magneton and $x=\frac{B}{B_{\text {crit } 1}}$.

The above solves exactly the problem of noninteracting electrons in a uniform magnetic field in full 3D space, by applying a procedure (of equilibrium relations) that is in a similar line of reasoning as in earlier sections, which is actually, the central line of approach that has been introduced in this article, to be used as a common tool for quite disparate problems (see also next section). It should also be noted that the above results are the limiting behaviours of the previous quasi-2D interface, when thickness becomes exceedingly large (it can be rigorously shown, for example, how the first critical field (5.5) arises from the rather involved analytical patterns of Section 4 in a complete analytical manner, demonstrating the consistency of our results).

Earlier works that follow different methods either do not give results for the total energy [27], or they mostly deal with a relativistic system [28], both of which are considerably involved in mathematics and do not quite reflect the basic Physics of the problem (i.e., the basic physical processes that are involved in the formation of the proper Fermi segments).

Below, the reader can find plots of all thermodynamic properties as functions of $B$. We should note again the continuity of energy and magnetisation but with the latter having cusps, leading to discontinuities and a highly nonlinear behaviour of susceptibility, something that we did not witness in the quasi-2D results of Section 4, where susceptibility was always piecewise constant.

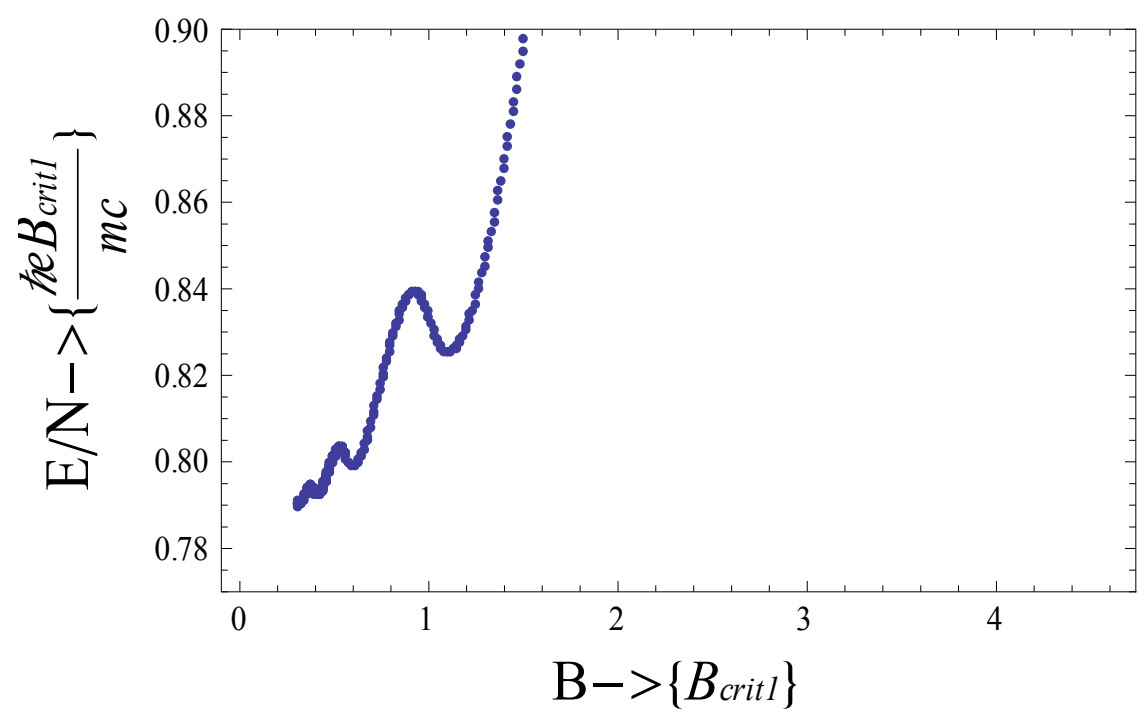



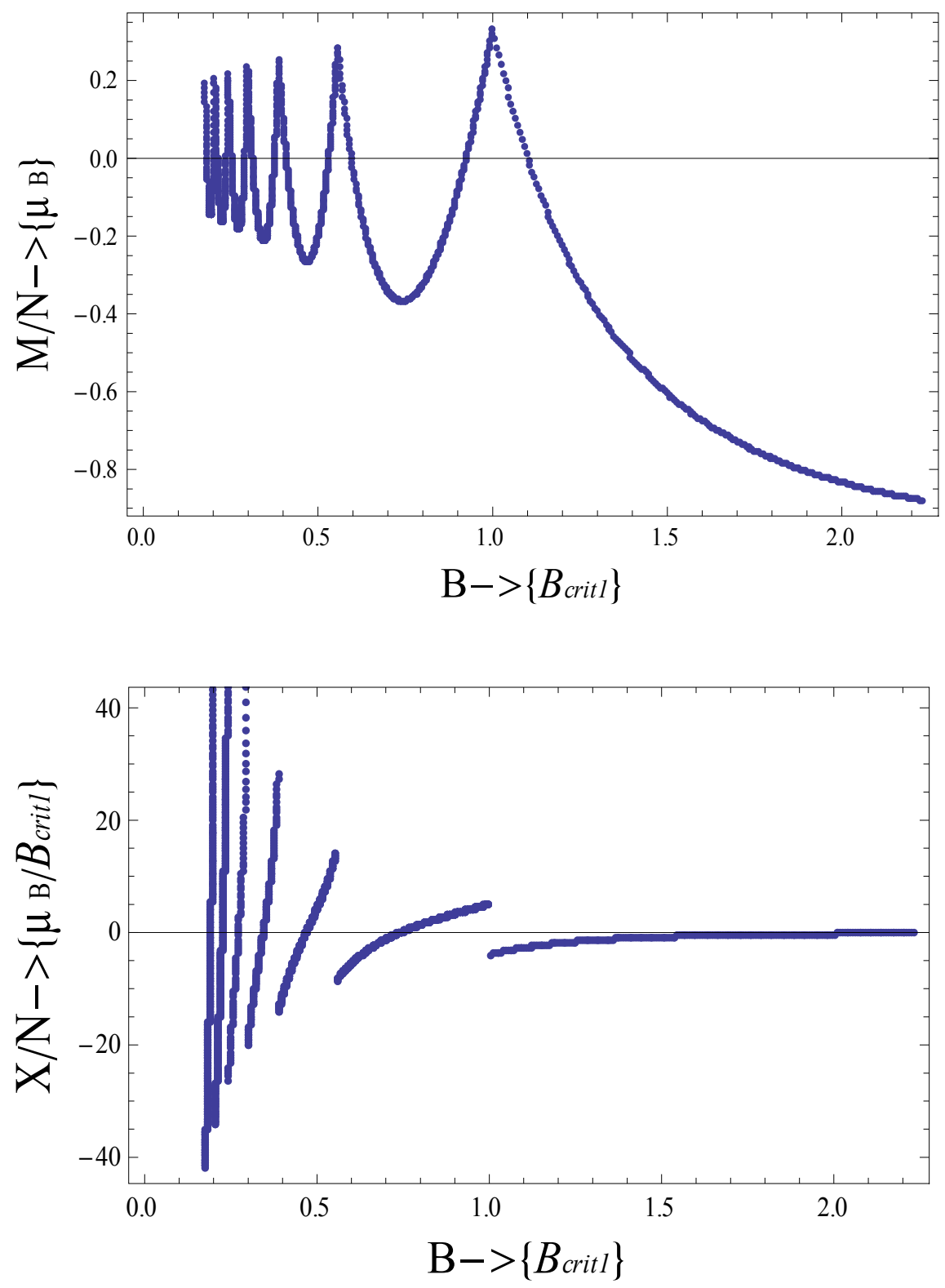

Fig. 5.3: Energy (in units of $\hbar e B_{c r i t 1} / m c$ ), Magnetisation (in units of $\mu_{\mathrm{B}}$ ) and Susceptibility (in units of $\left.\mu_{\mathrm{B}} / B_{\text {crit } 1}\right)$ as functions of $B$. Susceptibility, apart from being discontinuous, is highly nonlinear (compared with the quasi-2D cases of Section 4).

A final point must be made concerning Zeeman coupling. Analytical solutions involving imaginary parts of the Hurwitz zeta functions could also be obtained if the Zeeman term is included in the above calculations. In such a case, the energy spectrum (5.1) is modified as follows:

$$
\varepsilon_{n, k z}=\left(n+\frac{1}{2} \pm \frac{g^{*}}{4} \frac{m^{*}}{m}\right) \hbar \omega_{c}{ }^{*}+\frac{\hbar^{2} k_{z}{ }^{2}}{2 m^{*}}
$$

with $g^{*}$ the gyromagnetic ratio, $m^{*}$ the effective mass and $\omega_{c}{ }^{*}=e B / m^{*} c$ the effective cyclotron frequency. Although the problem is also completely solvable, we choose to only report the observation that, for sufficiently large $g^{*}$, we find a pronounced minimum in total energy as a function of $B$, i.e., when the gyromagnetic ratio is $g^{*}=1.5$ we obtain the behaviour shown in Fig. 5.4. Such behaviours originate from the interplay of QW, Zeeman and LL Physics in the full 3D problem and have not been reported earlier; as already noted in the 
Introduction, such minima might be important for the design of stable 3D quantum devices, i.e., in cases where the magnetic field can be self-consistently considered as self-generated.

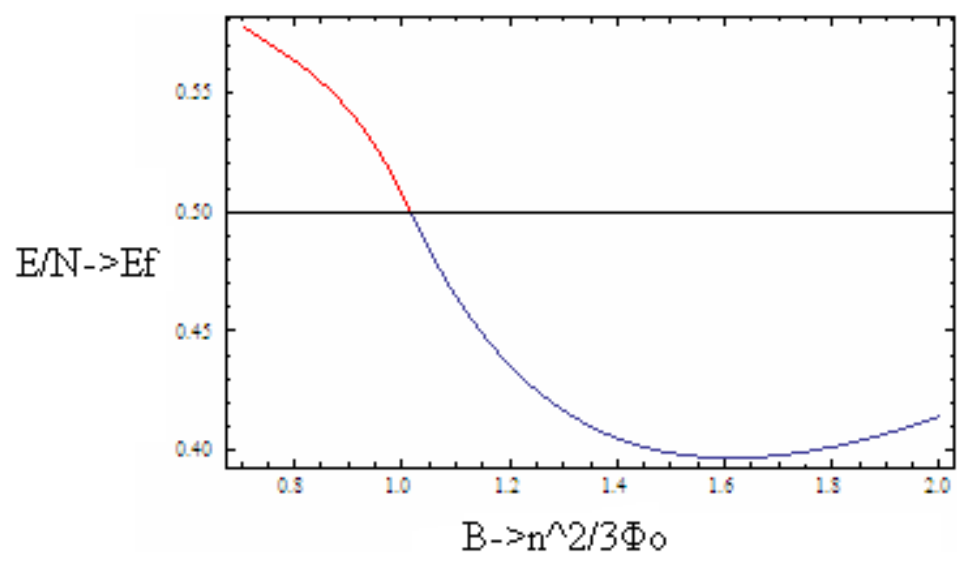

Fig. 5.4: Energy, as function of $B$, for $g^{*}=1.5$, for the first two windows of $B$. This minimum might be important in fabrication of small quantum devices.

\section{Relevance and applicability to the dimensionality crossover in Topological Insulators}

We have seen with an exact analytical solution and through a detailed analysis of energy interplay that the finite thickness is not as innocent as widely believed or implied. Its presence does not merely provide just another variable and just another label to the wavefunctions and energy spectra. Basically, this is because the Pauli principle can be circumvented momentarily at every step; these steps forming a sequence that leads to interesting and rather unpredictable behaviours. The method that we have followed for determining ground state thermodynamic magnetic quantities, such as the magnetisation, is not only exact but is also based on physically transparent arguments (on energy interplay and comparisons at the single-particle level, without the need of using the density of states). As a reward for this more physical approach, we have found, even for the above conventional systems that special values of thickness induce certain "internal transitions" (i.e., occurring at partial LL filling) that violate the standard de Haas-van Alphen periodicity; transitions that apparently have not been captured by other approaches. However, as an equally important reward, we should stress that because of its simplicity and universality in its line of reasoning, the same method could also be applied to other more involved systems of current interest, such as $3 \mathrm{D}$ strong topological insulators (such as $\mathrm{Bi}_{2} \mathrm{Se}_{3}$ ) and its dimensionality crossover to $2 \mathrm{D}$ topological insulators (such as $\mathrm{HgTe} / \mathrm{CdTe}$ wells).

To show this, we now briefly turn our attention to the well-known effective four-band model by Zhang et al. [29] that describes the low-energy behaviour of $\mathrm{Bi}_{2} \mathrm{Se}_{3}$. Such systems are described by a modified Dirac equation rather than the Schrodinger equation. This leads to very different wavefunctions (with nontrivial topological properties) and energy spectra, where the role of thickness is coupled to the 2D motion; however, the line of reasoning that we have developed and the general method that we have followed can still be applied in a similar manner. All one needs is essentially the one-particle spectrum, which incorporates the effect of thickness, even though this effect might be strongly coupled to the 2D degrees of freedom. Indeed, even for the coupled problem, one could determine the single-particle energy for the lowest value of a thickness-related quantum number, then determine the same for the next higher value of this quantum number and then study the comparison between the two energies - looking for cases of crossover between the two that might occur not too far from the $\Gamma$-point $\left(k_{x}=k_{y}=0\right)$. If there are also sufficient charge carriers that give a $k_{F}$ that is further away than the crossover point in $k$-space, this would be a strong indication that effects like the ones discussed above might also be present in these systems as well. We will carry out a quick calculation in the above spirit in the following but only in the thin-film limit, i.e., we will now have massive Dirac Fermions, which is even more relevant to our method because recently, it has been found [30] that for thin films there is a gap opening and the Fermi level does not fall in the gap; hence, surface carriers are present in the electron band with an estimated areal density of $\sim 5 \times 10^{16} \mathrm{~m}^{-2}$. In this thin-film limit, we will indeed find theoretical evidence of a clear crossover close to the $\Gamma$ point, inside the region of $k$-space where the Dirac equation is valid and with an estimated $k_{f}$ that is further away 
- something that shows that for these more exotic systems a more careful study of effects like the ones presented in the present work is probably needed.

One can start with the effective model that describes the bulk states near the $\Gamma$-point for bulk $\mathrm{Bi}_{2} \mathrm{Se}_{3}$ [29]. The Hamiltonian is given by:

$$
H(\vec{k})=\varepsilon_{o}(\vec{k}) I_{4 \times 4}+\left(\begin{array}{cccc}
M(\vec{k}) & -i A_{1} \partial_{z} & 0 & A_{2} k_{-} \\
-i A_{1} \partial_{z} & -M(\vec{k}) & A_{2} k_{-} & 0 \\
0 & A_{2} k_{+} & M(\vec{k}) & i A_{1} \partial_{z} \\
A_{2} k_{+} & 0 & i A_{1} \partial_{z} & -M(\vec{k})
\end{array}\right) .
$$

In a basis $\left|p 1_{z}^{+}, \uparrow\right\rangle,\left|p 2_{z}^{-}, \uparrow\right\rangle,\left|p 1_{z}^{+}, \downarrow\right\rangle,\left|p 2_{z}^{-}, \downarrow\right\rangle$ where $+(-)$ stands for even (odd) parity with

$$
\varepsilon_{o}(\vec{k})=C-D_{1} \partial_{z}^{2}+D_{2} k^{2}, M(\vec{k})=M+B_{1} \partial_{z}^{2}-B_{2} k^{2}, k_{ \pm}=k_{x} \pm i k_{y}, k^{2}=k_{x}^{2}+k_{y}^{2}
$$

with the model parameters having values:

$M=0.28 \mathrm{eV}, A_{1}=2.2 \mathrm{eV} \stackrel{\circ}{\mathrm{A}}, A_{2}=4.1 \mathrm{eV} \stackrel{o}{\mathrm{~A}}, B_{1}=10 \mathrm{eV} \stackrel{\circ}{\mathrm{A}^{2}}, B_{2}=56.6 \mathrm{eV} \stackrel{o}{\mathrm{~A}^{2}}, C=-0.0068 \mathrm{eV}, D_{1}=1.3 \mathrm{eV} \stackrel{o}{\mathrm{~A}^{2}}$, $D_{2}=19.6 \mathrm{eV} \stackrel{o}{\mathrm{~A}^{2}}$

and with a 4-component trial wavefunction:

$$
\Psi=\Psi_{\lambda} e^{\lambda z}
$$

(6.1) has been diagonalised [31] giving $\lambda_{\alpha}$ as functions of $E$ and $k$ [see (5) of [31]]. By inverting them, we obtain $E=E\left(\lambda_{\alpha}, k\right)$ and by focusing on the electron band, we obtain:

$$
E_{e l}^{\alpha=2}(\vec{k})=-0.0068+19.6 k^{2}-\frac{12.8305 \lambda_{2}^{2}}{\pi^{2}}-\sqrt{0.0784-14.886 k^{2}+3203.56 k^{4}+\frac{7.5 \lambda_{2}^{2}}{\pi^{2}}-\frac{11172.4 k^{2} \lambda_{2}^{2}}{\pi^{2}}+100 \lambda_{2}^{4}} .
$$

Although this problem must be treated numerically for a self-consistent determination of $E$ and $\lambda$ s, we can immediately check the thin-film limit, where it is found [31] that $\lambda=\mathrm{i} n_{z} \pi / d$. By plugging this into (6.3), we obtain the single-particle spectrum for the electron band as a function of $k$ for each $n_{z}$, namely:

$$
E_{e l}^{\alpha=2}(\vec{k})=-0.0068+19.6 k^{2}+\frac{12.8305 n_{z}^{2}}{d^{2}}-\sqrt{0.0784-14.886 k^{2}+3203.56 k^{4}-\frac{7.5 n_{z}^{2}}{d^{2}}+\frac{11172.4 k^{2} n_{z}^{2}}{d^{2}}+100 \frac{n_{z}^{4}}{d^{4}}} .
$$

Then, by using a value of $d=10 \mathrm{~nm}$ and plotting (6.4) for $n_{z}=1$ and $n_{z}=2$, we indeed find a crossover close to the $\Gamma$-point, as shown in Figs. 6.1-6.3. 


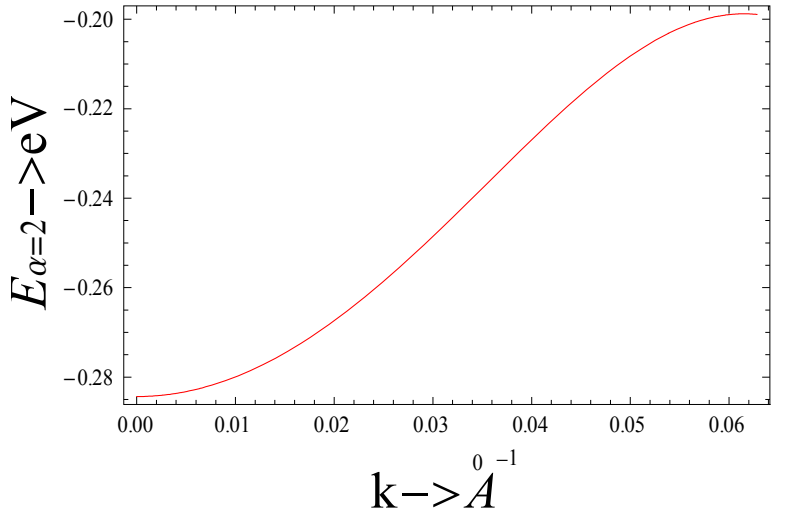

Fig. 6.1: $E_{e l}^{\alpha=2}(\vec{k})$ for $n_{z}=1$.

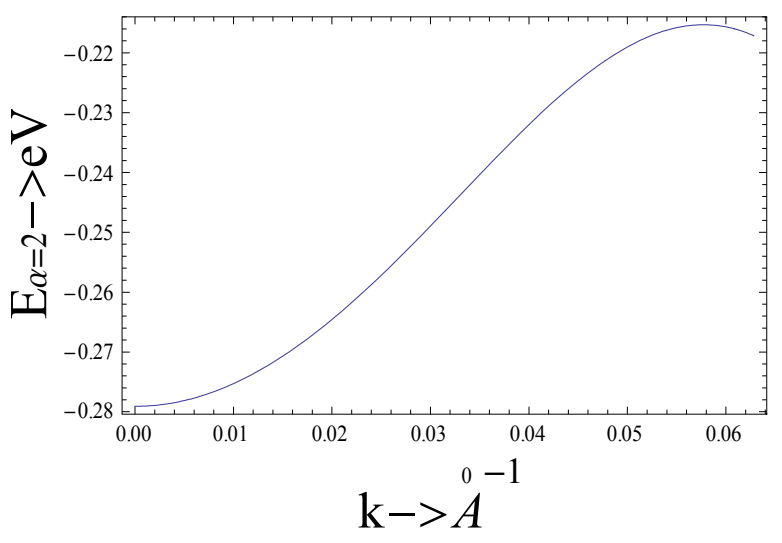

Fig. 6.2: $E_{e l}^{\alpha=2}(\vec{k})$ for $n_{z}=2$.

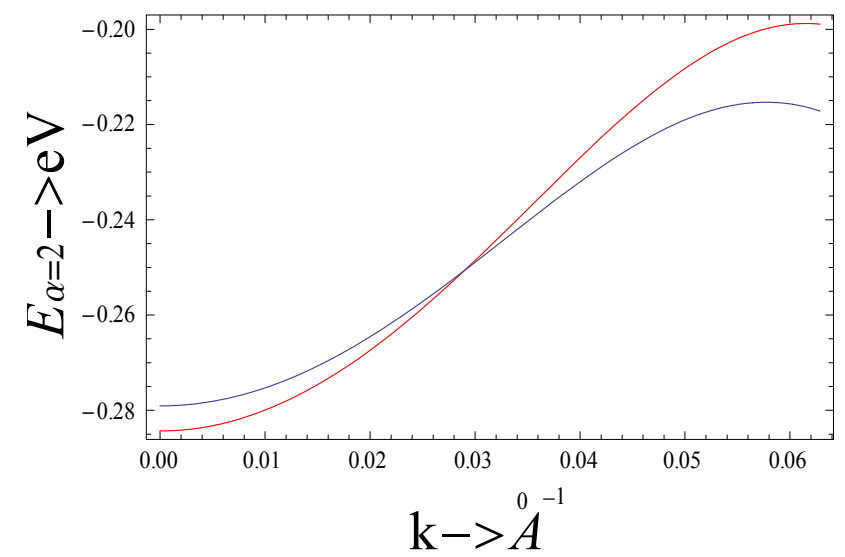

Fig. 6.3: $E_{e l}^{\alpha=2}(\vec{k})$ for $n_{z}=1$ and $n_{z}=2$ shown together

Moreover, note that, in analogy to Section 3, the occupational procedure is similar. For example, a Fermi wavevector for this band is now $k_{F}=\sqrt{\pi n_{A}}$ (with $n_{A}$ being the mean surface areal density) for a certain spin configuration. Then by using the estimate of density given above, a value of $k_{F}=0.04 \mathrm{~A}^{-1}$ is obtained, which is further on the right of the crossover point in Fig. 6.3, indicating that we have sufficient carriers that might exploit the crossover for internal transitions of the general type studied in this paper.

Independently, let us try to examine the first critical value of $d$ where the first transition occurs; however, in this quasi-2D topological insulator, we must now have (a generalisation of (3.4)):

$$
E_{e l}^{\alpha=2}\left(k=k_{F}, n_{z}=1\right)=E_{e l}^{\alpha=2}\left(k=0, n_{z}=2\right),
$$

or equivalently

$$
\begin{gathered}
-0.0068+19.6 k_{F}^{2}+\frac{12.8305}{d^{2}}-\sqrt{0.0784-14.886 k_{F}^{2}+3203.56 k_{F}^{4}-\frac{7.5}{d^{2}}+\frac{11172.4 k_{F}^{2}}{d^{2}}+100 \frac{1}{d^{4}}}= \\
-0.0068+\frac{12.8305 \times 4}{d^{2}}-\sqrt{0.0784-\frac{7.5 \times 4}{d^{2}}+100 \frac{16}{d^{4}}} .
\end{gathered}
$$


This equation determines the first critical value of $d$ in which the two-dimensional topological insulator starts becoming three-dimensional. By plugging in the estimated $k_{F}$ above, the solution of (6.6) gives $d=3.86 \mathrm{~nm}$; something that indicates that our tentative value of $d$ (of $10 \mathrm{~nm}$ ) is indeed in a region where interesting effects might be expected and that, generally speaking, strengthens the necessity for more careful treatment of this system.

\section{Conclusions}

An exact solution providing analytical expressions for the magnetic thermodynamic functions of an interface or film in a perpendicular magnetic film (with rigid walls) has been presented, in a picture of noninteracting electrons. Interactions were later taken into account by following the same method for the Landau $\Lambda$-levels in a picture of noninteracting Composite Fermions. The method used, different from standard density of states methods and grandcanonical and semi-classical approaches, is exact but also physically transparent at every step; hence, providing the possibility of application to more involved systems, such as 3D topological insulators, in which the thickness-related modes are strongly coupled to the planar motion. Even for conventional systems, it has been found that the finite thickness is not as innocent as widely believed or implied. Its presence does not merely provide just another variable and just another label to the wavefunctions and energy spectra. Basically, this is because the Pauli principle can be circumvented momentarily at every step; these steps forming a sequence that leads to interesting and rather unpredictable behaviours. The finite thickness has been found here to induce certain "internal transitions" (at partial Landau Level filling) of magnetisation that are not captured by earlier approaches and that violate the standard de Haas-van Alphen periodicities. The correctness of all these results has been tested against an independent exact analytical solution of the full 3D problem, which apparently, also leads to certain behaviours that have not been reported earlier. For topologically nontrivial systems, evidence that such effects might also be operative in the dimensionality crossover between 3D and 2D topological insulator wells has also been given. This suggests that the versatile method presented here needs to be carefully applied to such systems, a task that can be carried out numerically if the analytical patterns are too involved. This is something that is currently under investigation.

\section{References}

[1] M.Z. Hasan and C.L. Kane, Rev. Mod. Phys. 82(4), 3045 (2010)

[2] X.L. Qi and S.C. Zhang, Rev. Mod. Phys. 83(4), 1057 (2011)

[3] Y. Hatsugai, Phys. Rev. Lett. 71, 3697 (1993)

[4] D.J. Thouless, M. Kohmoto, P. Nightingale and M. den Nijs, Phys. Rev. Lett. 49, 405 (1982)

[5] M. Nakahara, Geometry, Topology and Physics, 2nd Edn. Taylor \& Francis (2003)

[6] D. Shoenberg, Magnetic Oscillations in Metals, Cambridge University Press (1984)

[7] H. Cao, J. Tian, I. Miotkowski, T. Shen, J. Hu, S. Qiao and Y.P. Chen, Phys. Rev. Lett. 108, 216803 (2012)

[8] Z.D. Kvon, E.B. Olshanetsky, D.A. Kozlov, E. Novik, N.N. Mikhailov and S.A. Dvoretsky, Fiz. Nizk. Temp. 37, 258 (2011) [Low Temp. Phys. 37, 202 (2011)]

[9] J. Nuebler, B. Friess, V. Umansky, B. Rosenow, M. Heiblum, K. von Klitzing and J. Smet, Phys. Rev. Lett. 108, 046804 (2012)

[10] Y. Liu, D. Kamburov, M. Shayegan, L.N. Pfeiffer, K.W. West and K.W. Baldwin, Phys. Rev. Lett. 107, 176805 (2011)

[11] M.R. Peterson, T. Jolicoeur and S. Das Sarma, Phys. Rev. B 78, 155308 (2008)

[12] K. Takei, H. Fang, B. Kumar, R. Kapadia, Q. Gao, M. Madsen, H.S. Kim, C.H. Liu, E. Plis, S. Krishna, H.A. Bechtel, J. Guo and A. Javey, Nano Lett., 11 (11), 5008 (2011)

[13] A. Ohtomo and H.Y. Hwang, Nature 427 (6973), 423 (2004)

[14] C.X. Liu, H. Zhang, B. Yan, X.L. Qi, T. Frauenheim, X. Dai, Z. Fang and S.C. Zhang, Phys. Rev. B 81, 041307(R) (2010)

[15] M.A. Wilde, M.P. Schwarz, C. Heyn, D. Heitmann, D. Grundler, D. Reuter and A.D. Wiecket, Phys. Rev. B 73, 125325 (2006)

[16] T. Chakraborty and P. Pietilainen, The Quantum Hall Effects, 2nd Edn. Springer (1995)

[17] V.M. Apalkov and T. Chakraborty, Phys. Rev. Lett., 107, 186801 (2011)

[18] S.K.F. Islam and T.K. Ghosh, Journ. Phys.: Condens. Mat. 24, 035302 (2012)

[19] X.F. Wang and P. Vasilopoulos, Phys. Rev. B 67, 085313 (2003)

[20] K. Moulopoulos and M. Aspromalli (unpublished); the variational proof is actually contained in an early Master's Thesis of M. Aspromalli ("Energy studies of quantal electrons inside a magnetic field: from astrophysical systems to semiconducting heterostructures", University of Cyprus (2004) (in Greek)) 
[21] M.C. Tringides, M. Jalochowski and E. Bauer, Phys. Today 60(4), 50 (2007)

[22] W.A. Atkinson and A.J. Slavin, Am. J. Phys. 76(12), 1099 (2008)

[23] V.D. Dymnikov, Fiz. Tverd. Tela 53, 847 (2011) [Phys. Solid State 53, 901 (2011)]

[24] S. Das Sarma and A. Pinczuk (Eds.), Perspectives in Quantum Hall Effects, Wiley-VCH (1997)

[25] J. Jain, Composite Fermions, Cambridge University Press (2007)

[26] D. Lai, Rev. Mod. Phys. 73, 629 (2001)

[27] Y.B. Suh, Ann. Phys. 94, 243 (1975)

[28] C.O. Dib and O. Espinosa, Nucl. Phys. B 612, 492 (2001)

[29] H. Zhang, C.X. Liu, X.L. Qi, X. Dai, Z. Fang and S.C. Zhang, Nat. Phys. 5, 438 (2009)

[30] A.A. Taskin, S. Sasaki, K. Segawa and Y. Ando, Phys. Rev. Lett. 109, 066803 (2012)

[31] W.Y. Shan, H.Z. Lu and S.Q. Shen, New Journ. Phys. 12, 043048 (2010) 